TRANSACTIONS OF THE

AMERICAN MATHEMATICAL SOCIETY

Volume 353, Number 11, Pages 4583-4622

S 0002-9947(01)02452-7

Article electronically published on July 3, 2001

\title{
THE BOTT-BOREL-WEIL THEOREM FOR DIRECT LIMIT GROUPS
}

\author{
LOKI NATARAJAN, ENRIQUETA RODRÍGUEZ-CARRINGTON, \\ AND JOSEPH A. WOLF
}

\begin{abstract}
We show how highest weight representations of certain infinite dimensional Lie groups can be realized on cohomology spaces of holomorphic vector bundles. This extends the classical Bott-Borel-Weil Theorem for finitedimensional compact and complex Lie groups. Our approach is geometric in nature, in the spirit of Bott's original generalization of the Borel-Weil Theorem. The groups for which we prove this theorem are strict direct limits of compact Lie groups, or their complexifications. We previously proved that such groups have an analytic structure. Our result applies to most of the familiar examples of direct limits of classical groups. We also introduce new examples involving iterated embeddings of the classical groups and see exactly how our results hold in those cases. One of the technical problems here is that, in general, the limit Lie algebras will have root systems but need not have root spaces, so we need to develop machinery to handle this somewhat delicate situation.
\end{abstract}

\section{INTRODUCTION}

The classical Bott-Borel-Weil Theorem [6] realizes representations of compact Lie groups as cohomology spaces of holomorphic vector bundles. Many of the geometric realizations of various kinds of representations are based on developments of this concept.

In this paper we carry the geometric idea of the Bott-Borel-Weil Theorem over to certain infinite-dimensional Lie groups. Those are the direct limits of finitedimensional compact Lie groups where the direct limit satisfies conditions (0.1), $(0.2)$ and (0.3). Those conditions are needed for the Borel-Weil Theorem 3.13. For the Bott-Borel-Weil Theorem we add the "cohomologically finite" condition of $\S 4$.

Several authors have obtained interesting results on direct limit groups, especially on their structure and representation theory. Some of the more topological results

Received by the editors May 6, 1997 and, in revised form, July 6, 1998 and April 26, 2000.

1991 Mathematics Subject Classification. Primary 222E30, 22E65; Secondary 22C05, 32C10, 46G20, 22E70.

Key words and phrases. Direct limit, direct limit Lie group, diagonal Lie algebra, diagonal embedding, Borel-Weil Theorem, Bott-Borel-Weil Theorem, direct limit representation, inverse limit representation, direct limit cohomology, inverse limit cohomology, infinite dimensional Lie group.

LN: research partially supported by NSF Grant DMS 9208303.

ERC: research partially supported by PSF-CUNY Grant 6-66386.

JAW: research partially supported by NSF Grants DMS 9321285 and DMS 9705709. 
are to be found in [8], [9, 10], 15], [16], 22, 23], 28], 29], 30] and 33]. Also see the citations in [27]. Those papers all consider direct limits in the category of topological groups. Some structural results on direct limits of finite dimensional Lie algebras can be found in [1], [2], [3], [4], [5], [12], [33] and [34].

We present the Bott-Borel-Weil Theorem as a holomorphic statement about the representations of the direct limit group. To do this we require a Lie group structure on this group. We introduced this structure in 24 and proved some of its properties in [25] and [26]. Helge Glöckner discovered [17] a couple of problems in these papers, and those points are corrected in Section 3 and Appendix A below. Now this Lie group structure theory is in good shape.

For the convenience of the reader, Appendices A and B contain precise definitions of our direct and inverse limit objects, including direct limit real Lie groups, direct limit complex manifolds and complex Lie groups, and direct limit complex analytic transformation groups.

Here are the standing definitions and hypotheses for this paper. Start with a countable strict direct system $\left\{G_{u, \alpha}, \phi_{\beta, \alpha}\right\}_{\alpha, \beta \in A}$ of compact connected Lie groups. Thus $(A, \leqq)$ is a countable directed set, i.e. a countable partially ordered set such that if $\alpha, \beta \in A$ there exists $\gamma \in A$ such that $\alpha \leqq \gamma$ and $\beta \leqq \gamma$. For each $\alpha$ in $A$, $G_{u, \alpha}$ is a compact connected Lie group. If $\alpha \leqq \beta$ in $A$, then $\phi_{\beta, \alpha}: G_{u, \alpha} \rightarrow G_{u, \beta}$ is a Lie group homomorphism. Further, we have the compatibility conditions (i) $\phi_{\alpha, \alpha}=\operatorname{id}_{G_{u, \alpha}}$ for each index $\alpha$ in $A$ and (ii) $\phi_{\gamma, \beta} \cdot \phi_{\beta, \alpha}=\phi_{\gamma, \alpha}$ whenever $\alpha \leqq \beta \leqq \gamma$ in $A$. The strictness condition is a technical condition needed in several places. Formally it says that $\phi_{\beta, \alpha}: G_{u, \alpha} \rightarrow \phi_{\beta, \alpha}\left(G_{u, \alpha}\right)$ is a diffeomorphism where the image carries the subspace topology from $G_{u, \beta}$. This condition implies that each $\phi_{\alpha}$ is a diffeomorphism onto its image. We also assume a certain growth condition (3.4b) to ensure the existence of the analytic structure on the limit group.

In the appendices the index set need not be countable. Appendix A gives sufficient conditions for the existence of the direct limit Lie group $G_{u}=\lim G_{u, \alpha}$. It is a Lie group in the sense of Definition A.8. Here our assumptions ensure that this direct limit exists. We denote by $\phi_{\alpha}: G_{u, \alpha} \rightarrow G_{u}$ the canonical map for each index $\alpha$ in $A$.

Now let $G_{\alpha}$ denote the connected complex Lie group with maximal compact subgroup $G_{u, \alpha}$ and let $\phi_{\beta, \alpha}: G_{\alpha} \rightarrow G_{\beta}$ denote the holomorphic extension of the direct limit system maps on the compact groups. As we show in Section 3, the direct limit $G=\underline{\varliminf} G_{\alpha}$ exists and is (isomorphic to) the complexification of $G_{u}$. The canonical maps $\phi_{\alpha}: G_{\alpha} \rightarrow G$ are the holomorphic extensions of the canonical maps $\phi_{\alpha}: G_{u, \alpha} \rightarrow G_{u}$. Strictness of $\left\{G_{u, \alpha}, \phi_{\beta, \alpha}\right\}$ implies strictness of $\left\{G_{\alpha}, \phi_{\beta, \alpha}\right\}$.

We write $\mathfrak{g}_{u, \alpha}$ for the Lie algebra of $G_{u, \alpha}$. Then $\left\{\mathfrak{g}_{u, \alpha}, d \phi_{\beta, \alpha}\right\}_{\alpha, \beta \in A}$ is a direct system of real Lie algebras and Lie algebra homomorphisms. Its direct limit $\mathfrak{g}_{u}=$ $\underline{\lim } \mathfrak{g}_{u, \alpha}$, in the category of topological Lie algebras, is the Lie algebra of $G_{u, \alpha}$. Similarly, the complexification $\mathfrak{g}_{\alpha}$ of $\mathfrak{g}_{u, \alpha}$ is the Lie algebra of $G_{\alpha},\left\{\mathfrak{g}_{\alpha}, d \phi_{\beta, \alpha}\right\}_{\alpha, \beta \in A}$ is a direct system of complex Lie algebras and Lie algebra homomorphisms, and $\mathfrak{g}=\lim _{\mathfrak{g}} \mathfrak{g}_{\alpha}$ is the Lie algebra of $G$.

We are going to define direct limits $\mathbb{E} \rightarrow X$ of holomorphic vector bundles $\mathbb{E}_{\alpha} \rightarrow X_{\alpha}$ over complex flags $X_{\alpha}=G_{\alpha} / Q_{\alpha}$ and prove an analogue of the BottBorel-Weil Theorem in that context. In order to set this up we assume that we have 
(0.1) $\mathfrak{h}_{\alpha} \subset \mathfrak{g}_{\alpha}$ : Cartan subalgebras in the Lie algebras $\mathfrak{g}_{\alpha}$ of the respective $G_{\alpha}$ such that

(i) $\mathfrak{h}_{u, \alpha}=\left(\mathfrak{h}_{\alpha} \cap \mathfrak{g}_{u, \alpha}\right)$ is a Cartan subalgebra of $\mathfrak{g}_{u, \alpha}$, for each $\alpha \in A$, and

(ii) $d \phi_{\beta, \alpha}\left(\mathfrak{h}_{\alpha}\right) \subset \mathfrak{h}_{\beta}$ whenever $\alpha \leqq \beta$ in $A$;

(0.2) positive root systems $\Delta_{\alpha}^{+}=\Delta^{+}\left(\mathfrak{g}_{\alpha}, \mathfrak{h}_{\alpha}\right)$ such that each $d \phi_{\beta, \alpha}$ maps every positive root space of $\mathfrak{g}_{\alpha}$ into the sum $\mathfrak{n}_{\beta}^{+}$of the positive root spaces of $\mathfrak{g}_{\beta}$, in other words such that $d \phi_{\beta, \alpha}\left(\mathfrak{n}_{\alpha}^{+}\right) \subset \mathfrak{n}_{\beta}^{+}$whenever $\alpha \leqq \beta$ in $A$;

(0.3) standard 1 parabolic subalgebras $\mathfrak{q}_{\alpha}=\mathfrak{l}_{\alpha}+\mathfrak{u}_{\alpha} \subset \mathfrak{g}_{\alpha}$ such that

(i) $d \phi_{\beta, \alpha}^{-1}\left(\mathfrak{q}_{\beta}\right)=\mathfrak{q}_{\alpha}$ whenever $\alpha \leqq \beta$ in $A$ and

(ii) $\mathfrak{g}_{u, \alpha} \cap \mathfrak{q}_{\alpha}$ is a compact real form of $\mathfrak{l}_{\alpha}$ for all $\alpha \in A$.

These three conditions are sufficient to prove many of the results in this paper, including the Borel-Weil (0-cohomology) Theorem. In the case of (0.1) through (0.3) where the $\mathfrak{q}_{\alpha}$ all are Borel subalgebras, as in [1], the $d \phi_{\beta, \alpha}$ are called triangular. We then have a triangular decomposition $\mathfrak{g}=\mathfrak{n}^{-}+\mathfrak{h}+\mathfrak{n}^{+}$, where $d \phi_{\alpha}\left(\mathfrak{n}_{\alpha}^{-}\right) \subset \mathfrak{n}^{-}$, $d \phi_{\alpha}\left(\mathfrak{h}_{\alpha}\right) \subset \mathfrak{h}$ and $d \phi_{\alpha}\left(\mathfrak{n}_{\alpha}^{+}\right) \subset \mathfrak{n}^{+}$.

To obtain the Bott-Borel-Weil (higher cohomology) Theorem in Section 4, technical considerations force us to modify the above conditions so that cohomology degrees stabilize for the system of homogeneous holomorphic vector bundles under consideration. This is done in $\S 4$ with the notions of "cohomological finiteness" and "classical cohomological finiteness" for the highest weight of the representation of the isotropy subgroup on the fiber. In order to carry this out, we introduce an appropriate notion of Weyl group $W(\mathfrak{g}, \mathfrak{h})$ and of finite Weyl group $W_{F}(\mathfrak{g}, \mathfrak{b}, \mathfrak{h})$. The latter depends on the choice of Borel subalgebra $\mathfrak{b}$ with $\mathfrak{h} \subset \mathfrak{b} \subset \mathfrak{g}$, in other words on the choice of complex structure on the limit flag manifold on which our BottBorel-Weil Theorem is formulated. The finite Weyl group allows us to specify the cohomology degrees of our representations. The necessity of locating $W_{F}(\mathfrak{g}, \mathfrak{b}, \mathfrak{h})$ within $W(\mathfrak{g}, \mathfrak{h})$ is just one of many surprising new technical problems that must be considered whenever one studies any moderately general class of reductive direct limit Lie groups and Lie algebras. In fact we can say with confidence that the present study has provided us with an adequate supply of technical surprises, but that the result was worth the effort.

Ivan Dimitrov noticed [1] a problem with our original treatment of cohomological finiteness in the case where the limit algebra does not have a root space decomposition, and we thank him for useful conversations on this matter. This situation is also addressed in [13] and [14].

We apply our machinery to diagonal embedding direct limit systems in $\S 5$, and to standard, Escher and Warhol direct limit systems in $\S 6$. The diagonal direct limit systems themselves break into two sharply distinct classes, the "classical" ones where the limit algebra has a root space decomposition, and the "non-classical" ones where it does not. The classical diagonal direct limit algebras are also known as "root-reductive" diagonal direct limit Lie algebras. In the classical case the notions of cohomological finiteness and classical cohomological finiteness coincide and the Bott-Borel-Weil Theorem leads to highest weight representations. In the nonclassical case, classical cohomological finiteness only occurs in the setting that leads

\footnotetext{
${ }^{1}$ Here "standard parabolic subalgebra" means that the nilradical $\mathfrak{u}_{\alpha}$ is spanned by negative root spaces, that the Levi complement $\mathfrak{l}_{\alpha}$ contains the Cartan subalgebra $\mathfrak{h}_{\alpha}$, and that there is a subset $\Phi_{\alpha}$ of the simple root system $\Psi\left(\mathfrak{g}_{\alpha}, \mathfrak{h}_{\alpha}\right) \subset \Delta^{+}\left(\mathfrak{g}_{\alpha}, \mathfrak{h}_{\alpha}\right)$ such that $\Delta\left(\mathfrak{l}_{\alpha}, \mathfrak{h}_{\alpha}\right)$ consists of all roots that are linear combinations of elements of $\Phi_{\alpha}$.
} 
to the Borel-Weil Theorem (cohomology in degree zero), but there are many cohomologically finite bundles, and the Bott-Borel-Weil Theorem constructs a number of new representations that do not seem to be highest weight representations. This is a new phenomenon, not yet well understood, and the classification of these representations presents some serious combinatorial challenges.

In $\S 7$ we define a notion of coherent root order. When we have a coherent root order we characterize the finite Weyl group as the $\operatorname{limit} \underset{\mathrm{lim}}{\longrightarrow} W_{\alpha}$ of the Weyl groups at finite stages. The notion of coherent root order is restrictive, but when it applies it is easy to use, and we see exactly when it applies to diagonal direct limits. In $\S 8$ we combine the examples of $\S 5$ and the considerations of $\S 7$ to put together a general characterization of the finite Weyl group $W_{F}(\mathfrak{g}, \mathfrak{b}, \mathfrak{h})$. That characterization tells us exactly when our Bott-Borel-Weil Theorem gives a precise quantitative result.

Let $Q_{\alpha} \subset G_{\alpha}$ denote the parabolic subgroup of $G_{\alpha}$ with Lie algebra $\mathfrak{q}_{\alpha}$ and define $X_{\alpha}$ to be the complex flag manifold $G_{\alpha} / Q_{\alpha}$. Combining $(0.1),(0.2)$ and (0.3) we obtain injective holomorphic maps $\phi_{\beta, \alpha}: X_{\alpha} \rightarrow X_{\beta}$ for $\alpha \leqq \beta$ in $A$. It is easy to see that the direct system $\left\{X_{\alpha}, \phi_{\beta, \alpha}\right\}$ is strict and defines a complex manifold $X=\lim X_{\alpha}$. Similarly we will see that the maps $G_{\alpha} \times X_{\alpha} \rightarrow X_{\alpha}$ define maps $G_{\alpha} \times X \rightarrow X$, and then $G \times X \rightarrow X$, and that $G \times X \rightarrow X$ is a holomorphic Lie transformation group. Further we will see that $Q=\lim Q_{\alpha}$ sits naturally as a closed complex Lie subgroup of $G$, and that $X=G / Q$ is a complex homogeneous space for $G$. Thus we arrive at the key geometric ingredients for our direct limit Borel-Weil and Bott-Borel-Weil Theorems in the holomorphic category.

There is also a Bott-Borel-Weil theory [14] in the algebraic category, direct limits of reductive linear algebraic groups (ind-groups). It is quite different from the holomorphic category Bott-Borel-Weil theory developed here, but there has been some interchange of ideas between [14] and the present paper.

\section{The Bundles}

As in the Introduction, we fix a strict direct system $\left\{G_{u, \alpha}, \phi_{\beta, \alpha}\right\}_{\alpha, \beta \in A}$ of compact connected Lie groups. $G_{\alpha}$ is the connected complex Lie group with maximal compact subgroup $G_{u, \alpha}$, the $\phi_{\beta, \alpha}: G_{\alpha} \rightarrow G_{\beta}$ are the holomorphic extensions of the direct limit system maps on the compact groups, $\left\{G_{\alpha}, \phi_{\beta, \alpha}\right\}$ is the corresponding strict direct system of complex connected Lie groups, and $G=\lim _{\longrightarrow} G_{\alpha}$ is its direct limit. We assume the Operator Norm Growth Condition $(3.4 \mathrm{~b}) \overrightarrow{\text { so }}$ that $G_{u}=\lim _{\longrightarrow} G_{u, \alpha}$ is a real Lie group and $G=\lim _{\longrightarrow} G_{\alpha}$ is a complex Lie group. We also assume $(0.1),(0.2)$ and $(0.3)$ from the Introduction so that we can deal with the ingredients of the Borel-Weil and Bott-Borel-Weil Theorems.

In general, if $\mathfrak{f}_{u, \alpha} \subset \mathfrak{g}_{u, \alpha}$ is a subspace, then $\mathfrak{f}_{\alpha} \subset \mathfrak{g}_{\alpha}$ denotes its complexification. If $\mathfrak{f}_{\alpha} \subset \mathfrak{g}_{\alpha}$ is a subspace invariant under the complex conjugation $\xi \mapsto \bar{\xi}$ of $\mathfrak{g}_{\alpha}$ over $\mathfrak{g}_{u, \alpha}$, then $\mathfrak{f}_{u, \alpha}$ denotes its real form $\mathfrak{f}_{\alpha} \cap \mathfrak{g}_{u, \alpha}$.

$Q_{\alpha} \subset G_{\alpha}$ is the parabolic subgroup with Lie algebra $\mathfrak{q}_{\alpha}=\mathfrak{l}_{\alpha}+\mathfrak{u}_{\alpha}$. Here $\mathfrak{u}_{\alpha}$ is the nilradical and $\mathfrak{l}_{\alpha}$ is the Levi component that contains $\mathfrak{h}_{\alpha}$. Thus $Q_{\alpha}=L_{\alpha} U_{\alpha}$ semidirect product, $L_{u, \alpha}=G_{u, \alpha} \cap Q_{\alpha}=G_{u, \alpha} \cap L_{\alpha}$ is a compact real form of $L_{\alpha}$, and $L_{u, \alpha}$ is the centralizer in $G_{u, \alpha}$ of the torus

$$
T_{u, \alpha}:=\text { identity component of the center of } L_{u, \alpha} \text {. }
$$

The limit group $Q=\lim _{\alpha} Q_{\alpha}$ sits naturally as a closed subgroup

$$
Q=\{g \in G \mid \operatorname{Ad}(g) \mathfrak{q}=\mathfrak{q}\}
$$


where $\mathfrak{q}=\lim _{\longrightarrow} \mathfrak{q}_{\alpha}$ of $G$. Since $G$ has the direct limit topology, i.e., a subset $U$ is open in $G$ if and only if each of its pullbacks $\phi_{\alpha}^{-1}(U)$ is open in the corresponding $G_{\alpha}$, and since each $\phi_{\alpha}^{-1}(Q)=Q_{\alpha}$ is closed, the subgroup $Q \subset G$ is closed in $G$.

The following basic construction depends on the fact that here we use the (naïve) direct limit topologies on $G_{u}$, on $G$, on $X$, and on $Q$. See [24] and Appendix A.

1.1. Proposition. Let $G=\lim G_{\alpha}$ and $X=\lim X_{\alpha}$ carry their respective direct limit complex structures: if $\vec{U}$ is an open subset in the direct limit topology, then a function $f: U \rightarrow \mathbb{C}$ is holomorphic if and only if each $f \cdot \phi_{\alpha}: \phi_{\alpha}^{-1}(U) \rightarrow \mathbb{C}$ is holomorphic. Let $x_{\alpha}$ denote the base point $1 Q_{\alpha}$ in $X_{\alpha}$ and let $x=\phi_{\alpha}\left(x_{\alpha}\right)$, independent of choice of $\alpha$, the corresponding point of $X$.

(1) The holomorphic group actions $t_{\alpha}: G_{\alpha} \times X_{\alpha} \rightarrow X_{\alpha}$ define a holomorphic group action $t: G \times X \rightarrow X$ as follows. Given $\alpha$ there is a natural holomorphic group action $t_{\alpha}: G_{\alpha} \times X \rightarrow X$ by $t_{\alpha}\left(g_{\alpha},\left[x_{\beta}\right]\right)=\left[\phi_{\beta, \alpha}\left(g_{\alpha}\right)\left(x_{\beta}\right)\right]$ for $\beta \geqq \alpha$, and $t=\lim _{\alpha} t_{\alpha}$ maps $G \times X \rightarrow X$.

(2) $G \times X=\lim _{\longrightarrow}\left(G_{\alpha} \times X_{\alpha}\right)$ in the appropriate category of complex manifolds.

(3) $Q$ is a closed complex analytic subgroup of $G$.

(4) $X=G / Q$ as complex quotient manifold as follows. $Q=\{g \in G \mid t(g, x)=x\}$, so $g \mapsto t(g, x)$ defines a one-to-one map of $G / Q$ onto $X$. Let $p: G \rightarrow X$ denote the projection, $p(g)=t(g, x)$. If $U \subset X$ is an open set, then a function $f: U \rightarrow \mathbb{C}$ is holomorphic if and only if $f \cdot p: p^{-1}(U) \rightarrow \mathbb{C}$ is holomorphic.

A quick look at the definitions of $Q$ and the $Q_{\alpha}$ shows

1.2. Lemma. $Q$ is the semidirect product $L U$ of closed complex subgroups where

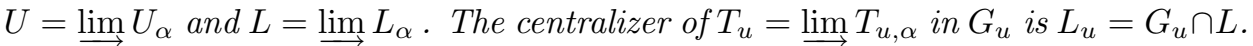
The $\overrightarrow{\text { map }} g \mapsto g Q$ sends $G_{u}$ onto $X$ and realizes $\vec{X}=G_{u} / L_{u}$ as real analytic manifolds.

Fix a compatible family of representations $\tau_{\alpha}$ of the $L_{\alpha}$. Thus for each index $\alpha$ we have a locally convex reflexive topological vector space $E_{\alpha}$ and a strongly continuous representation $\tau_{\alpha}$ of $L_{\alpha}$ on $E_{\alpha}$, and for indices $\alpha \leqq \beta$ we have continuous linear maps $\eta_{\beta, \alpha}: E_{\alpha} \rightarrow E_{\beta}$ whenever $\beta \geqq \alpha$, such that $\left\{E_{\alpha}, \eta_{\beta, \alpha}\right\}_{\alpha, \beta \in A}$ is a strict direct system and the diagrams

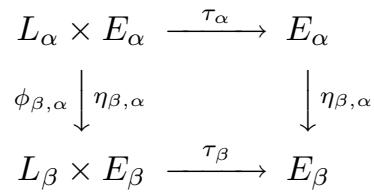

commute. In the language of Appendix A, the $\tau_{\alpha}$ form a compatible family of representations, so we can consider the direct limit representation $\tau=\lim \tau_{\alpha}$ of $L$ on $E=\lim E_{\alpha}$. We require the maps $\tau_{\alpha}: L_{\alpha} \times E_{\alpha} \rightarrow E_{\alpha}$ to be holomorphic because we are going to use the $E_{\alpha}$ as typical fibers in homogeneous holomorphic vector bundles. Then $\tau=\varliminf_{\alpha} \tau_{\alpha}$ extends from $L$ to $Q$ by $\tau(\ell u)=\tau(\ell)$.

Strictness of the direct system $\left\{E_{\alpha}, \eta_{\beta, \alpha}\right\}_{\alpha, \beta \in A}$ is automatic if the $E_{\alpha}$ are finite dimensional and the $\eta_{\beta, \alpha}$ are injective, or if the $E_{\alpha}$ are Hilbert spaces and the $\eta_{\beta, \alpha}$ are unitary injections. In $\S 3$ we will construct particular sorts of maps $\eta_{\beta, \alpha}$ using the Cartan highest weight theory.

1.4. Lemma. The topological vector bundle $G \times{ }_{Q} E=\mathbb{E} \rightarrow X$ over $X=G / Q$, associated to the action $\tau$ of $Q$ on $E$, has a unique structure of $G$-homogeneous 
holomorphic vector bundle. It is the direct limit of the bundles $G_{\alpha} \times_{Q_{\alpha}} E_{\alpha}=\mathbb{E}_{\alpha} \rightarrow$ $X_{\alpha}$ with the direct limit system maps

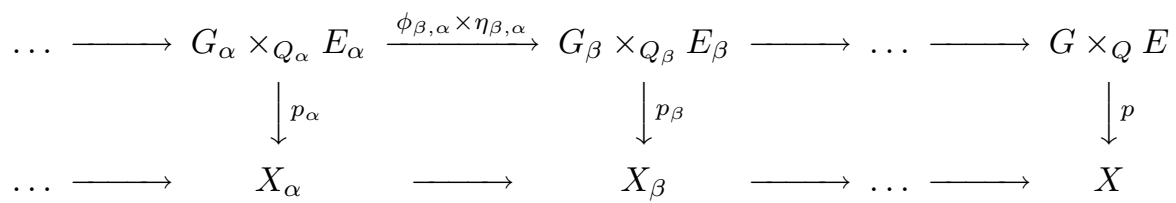

Proof. We first look at the principal bundle $Q$-bundle $G \rightarrow X$. Consider the Lie algebra decompositions $\mathfrak{g}_{\alpha}=\mathfrak{q}_{\alpha}+\mathfrak{q}_{\alpha}^{-n}$ where $\mathfrak{q}_{\alpha}^{-n}$ is the sum of root spaces not contained in $\mathfrak{q}_{\alpha}$, and represents the holomorphic tangent space of $X_{\alpha}$. If $\alpha \leqq \beta$ then $d \phi_{\beta, \alpha}\left(\mathfrak{q}_{\alpha}^{-n}\right) \subset \mathfrak{q}_{\beta}^{-n}$. Now $\mathfrak{q}^{-n}=\lim _{\mathfrak{\alpha}} \mathfrak{q}_{\alpha}^{-n}$ is well-defined. By hypothesis there exists an open neighborhood $\mathcal{N}$ of 0 in the limit Lie algebra which is mapped diffeomorphically by $\exp _{G}$ onto an open neighborhood of 1 in $G$. Now (i) $\exp _{G}$ maps $\mathfrak{q}^{-n} \cap \mathcal{N}$ diffeomorphically onto an open neighborhood of $1 Q=x \in X$ and (ii) $\nu: \exp _{G}(\xi) Q \mapsto \exp _{G}(\xi)$ is a holomorphic local section of $G \rightarrow X$ over that neighborhood. Note $\nu=\underline{\longrightarrow} \nu_{\alpha}$ where $\nu_{\alpha}: \exp _{G_{\alpha}}(\xi) Q_{\alpha} \mapsto \exp _{G_{\alpha}}(\xi)$ is a holomorphic local section of $G_{\alpha} \rightarrow X_{\alpha}$ over the neighborhood $\exp _{G_{\alpha}}\left(\mathfrak{q}_{\alpha}^{-n} \cap d \phi_{\alpha}^{-1}(\mathcal{N})\right) Q_{\alpha}$. Now $\mathbb{E}=\underline{\lim } \mathbb{E}_{\alpha}$ with properties as asserted.

We denote the bundle of Lemma 1.4 by $\mathbb{E}=\varliminf_{\longrightarrow} \mathbb{E}_{\alpha}$ and its strong topological dual by $\mathbb{E}^{*} \rightarrow X$.

\section{The Sheaves}

In this section we consider the inverse system $\left\{E_{\alpha}^{*}, \eta_{\beta, \alpha}^{*}\right\}$, where $E_{\alpha}^{*}$ is the strong topological dual space to $E_{\alpha}$ and $\eta_{\beta, \alpha}^{*}$ is dual to $\eta_{\beta, \alpha}: E_{\alpha} \rightarrow E_{\beta}$. We use it to map germs, local sections, and global sections, of the associated homogeneous holomorphic vector bundles. The corresponding maps on sheaves of germs of holomorphic sections,

$$
\zeta_{\beta, \alpha}: \mathcal{O}\left(\mathbb{E}_{\beta}^{*}\right) \rightarrow \mathcal{O}\left(\mathbb{E}_{\alpha}^{*}\right) \text { for } \beta \geqq \alpha,
$$

define a $G$-homogeneous inverse limit sheaf $\mathcal{O}\left(\mathbb{E}^{*}\right) \rightarrow X$, and will lead to theorems of Bott-Borel-Weil type.

Recall the definition of the inverse limit sheaf $\mathcal{O}\left(\mathbb{E}^{*}\right)=\varliminf_{\lfloor}\left\{\mathcal{O}\left(\mathbb{E}_{\beta}^{*}\right), \zeta_{\beta, \alpha}\right\}$. First, view the $\mathcal{O}\left(\mathbb{E}_{\beta}^{*}\right) \rightarrow X_{\beta}$ as sheaves over $X$ : if $U$ is open in $X$ then $\Gamma(U)=$ $\Gamma\left(\phi_{\beta}^{-1}(U) ; \mathcal{O}\left(\mathbb{E}_{\beta}^{*}\right)\right)$ defines a complete presheaf on $X$, and $\varliminf_{\mathcal{O}} \mathcal{O}\left(\mathbb{E}_{\beta}^{*}\right) \rightarrow X$ is the associated sheaf. See [20, Ch. II, Prop. 9.2]. Second, this respects the notion of holomorphic, for an exercise in the definitions proves

2.2. Lemma. Let $U_{\beta} \subset G_{\beta}$ be an open subset. Let $s_{\beta}: U_{\beta} \rightarrow E_{\beta}^{*}$ be a continuous local section of $\mathbb{E}_{\beta}^{*} \rightarrow X_{\beta}$. Set $U_{\alpha}=\phi_{\beta, \alpha}^{-1}\left(U_{\beta}\right) \subset G_{\alpha}$ where $\beta \geqq \alpha$. Define $s_{\alpha}=\zeta_{\beta, \alpha}\left(s_{\beta}\right): U_{\alpha} \rightarrow E_{\alpha}^{*}$ by $s_{\alpha}(g)=\eta_{\beta, \alpha}^{*}\left(s_{\beta}\left(\phi_{\beta, \alpha}(g)\right)\right)$. Then $s_{\alpha}$ is a continuous local section of $\mathbb{E}_{\alpha}^{*} \rightarrow X_{\alpha}$. If $s_{\beta}$ is holomorphic then $s_{\alpha}$ is holomorphic.

(The point is that $\mathbb{E}_{\alpha} \rightarrow \mathbb{E}_{\beta}$ covers $X_{\alpha} \rightarrow X_{\beta}$ as in (1.5) and the maps $\phi_{\beta, \alpha}$ : $X_{\alpha} \rightarrow X_{\beta}$ are holomorphic.) Third, this definition is consistent with that of Lemma 1.4 : 
2.3. Lemma. The inverse limit sheaf $\lim _{\{}\left\{\mathcal{O}\left(\mathbb{E}_{\alpha}^{*}\right), \zeta_{\beta, \alpha}\right\} \rightarrow X$ is canonically and $G$-equivariantly isomorphic to the sheaf $\mathcal{O}_{\left(\mathbb{E}^{*}\right)} \rightarrow X$ of germs of holomorphic sections of $\mathbb{E}^{*} \rightarrow X$.

Let $\mathcal{S}_{\alpha} \rightarrow X$ denote the sheaf $\mathcal{O}\left(\mathbb{E}_{\alpha}^{*}\right) \rightarrow X_{\alpha}$ viewed as a sheaf on $X$ with zero stalk at each point of $X \backslash X_{\alpha}$, as in the definition of the inverse limit sheaf $\left.\underset{\lim }{\longleftarrow}\left\{\mathbb{E}_{\beta}^{*}\right), \zeta_{\beta, \alpha}\right\}$ over $X$. We want to check that cohomology commutes with these inverse limits, that is, $\lim H^{q}\left(X ; \mathcal{S}_{\alpha}\right)=H^{q}\left(X, \lim _{L} S_{\alpha}\right)$. The result2 is

2.4. Proposition. There is a natural $G$-equivariant isomorphism from the cohomology $H^{q}\left(X ; \mathcal{O}\left(\mathbb{E}^{*}\right)\right)$ of the inverse limit onto the inverse limit

$$
\varliminf\left\{H^{q}\left(X_{\alpha} ; \mathcal{O}\left(\mathbb{E}_{\alpha}^{*}\right)\right), H^{q} \zeta_{\beta, \alpha}\right\}
$$

of the cohomologies.

Proof. We apply [19, Chapter I, Theorem 4.5] with the global section functor $\Gamma$ in place of $T$, so its derived functors are our sheaf cohomologies. Use (0.2) to align Bruhat decompositions of the $G_{\alpha}$ so that we have a neighborhood basis of the base point $x \in X$ whose $\phi_{\alpha}$-inverse image is a neighborhood basis of the base point $x_{\alpha} \in X_{\alpha}$ in which every element is a Stein submanifold. Then conditions (a) and (b) of [19, Chapter I, Theorem 4.5] are satisfied, so we have (equivariant) exact sequences

$$
0 \rightarrow \varliminf^{(1)} H^{q-1}\left(X ; \mathcal{S}_{\alpha}\right) \rightarrow H^{q}\left(X ; \mathcal{O}\left(\mathbb{E}^{*}\right)\right) \rightarrow \underline{\lim } H^{q}\left(X ; \mathcal{S}_{\alpha}\right) \rightarrow 0
$$

where $\varliminf^{(1)}$ is the first right derived functor of the $\varliminf_{\text {lim }}$ functor. Thus the proof of Proposition 2.4 is reduced to the proof that the $\varliminf^{(1)} H^{q-1}\left(X ; \mathcal{S}_{\alpha}\right)=0$. And for that it suffices [19, Chapter I, Corollary 4.3] to check the Mittag-Leffler condition

for each $\alpha$ the filtration of $H^{q-1}\left(X ; \mathcal{S}_{\alpha}\right)$

by the $\zeta_{\beta, \alpha} H^{q-1}\left(X ; \mathcal{S}_{\beta}\right)$ is eventually constant.

But that is immediate from finite dimensionality of the

$$
H^{q-1}\left(X ; \mathcal{S}_{\alpha}\right) \cong H^{q-1}\left(X_{\alpha} ; \mathcal{O}\left(\mathbb{E}_{\alpha}^{*}\right)\right) \text {. }
$$

\section{The Borel-Weil Theorem}

We now suppose that the representations $\tau_{\alpha}: L_{\alpha} \times E_{\alpha} \rightarrow E_{\alpha}$ of (1.3) are irreducible, are finite dimensional, and are given by a coherent system of lowest weights. In other words $\tau_{\alpha}$ is the holomorphic extension of an irreducible representation $\tau_{u, \alpha}=\left.\tau_{\alpha}\right|_{L_{u, \alpha}}$ of the compact real form $L_{u, \alpha}$, and we have

$\lambda \in \mathfrak{h}^{*}=\left(\varliminf_{\lim } \mathfrak{h}_{\alpha}\right)^{*}$ such that, for each $\alpha, \tau_{\alpha}$ has lowest weight $-\lambda_{\alpha}=-d \phi_{\alpha}^{*}(\lambda)$.

Here $\lim _{\mathfrak{h}} \mathfrak{h}_{\alpha}$ has the direct limit topology and its dual $\mathfrak{h}^{*}$ is the topological dual, consisting of continuous linear functionals on $\lim \mathfrak{h}_{\alpha}$. Note that the dual representation $\tau_{\alpha}^{*}: L_{\alpha} \times E_{\alpha}^{*} \rightarrow E_{\alpha}^{*}$ has highest weight $\lambda_{\alpha}=d \phi_{\alpha}^{*}(\lambda)$. Of course one has representations $\tau_{\alpha}$ as in (3.1) precisely when $\lambda \in \mathfrak{h}^{*}$ such that each $\lambda_{\alpha}$ is $\Delta_{\alpha}^{+-}$ dominant and $L_{\alpha}$-integral.

\footnotetext{
${ }^{2}$ We thank the referee for a sharp improvement in our original treatment of this matter.
} 
We suppose further that the compatible linear maps $\eta_{\beta, \alpha}: E_{\alpha} \rightarrow E_{\beta}$ of (1.3) are given by a choice of lowest weight vectors $0 \neq v_{\alpha}=v_{-\lambda_{\alpha}} \in E_{\alpha}$. In other words

$$
\eta_{\beta, \alpha}\left(d \tau_{\alpha}(\Xi) v_{\alpha}\right)=d \tau_{\beta}\left(d \phi_{\beta, \alpha}(\Xi)\right) v_{\beta} \text { for all } \Xi \in \mathcal{U}\left(\mathfrak{l}_{\alpha}\right) .
$$

Here $\mathcal{U}\left(\mathfrak{l}_{\alpha}\right)$ is the universal enveloping algebra of $\mathfrak{l}_{\alpha}$. Note that (3.2) specifies the maps $\eta_{\beta, \alpha}$ because $E_{\alpha}=d \tau_{\alpha}\left(\mathcal{U}\left(\mathfrak{l}_{\alpha}\right)\left(v_{\alpha}\right)\right)$. These maps are well-defined, are injective, and satisfy the compatibility conditions of (1.3).

3.3. Lemma. Each $E_{\alpha}$ has a Hilbert space structure such that $\tau_{u, \alpha}=\left.\tau_{\alpha}\right|_{L_{u, \alpha}}$ is unitary, and we may choose the lowest weight vectors $v_{\alpha} \in E_{\alpha}$ such that $\eta_{\beta, \alpha}$ : $E_{\alpha} \rightarrow E_{\beta}$ is a unitary injection for $\alpha \leqq \beta$.

Proof. The $L_{u, \alpha}$-invariant Hilbert space structures exist because $L_{u, \alpha}$ is compact. Fix them, and choose the $v_{\alpha}$ to be unit vectors. The hermitian inner product on $E_{\alpha}$ is proportional to the pull-back of the inner product on $E_{\beta}$ because both are invariant under the irreducible action of $L_{u, \alpha}$, and the proportionality factor is 1 because $\eta_{\beta, \alpha}\left(v_{\alpha}\right)=v_{\beta}$.

We can now use this compatible family of Hilbert structures on the $E_{\alpha}$ to define a hermitian inner product on $E$ thus realizing it as a pre-Hilbert space. For details see the proof of Proposition B.1 of Appendix B.

We must recall a few notions concerning direct limit representations from [24], 25] and [26], especially [24, §2], to formulate some basic notions we need for our direct limit representations.

Consider a strict direct system $\left\{J_{\alpha}, \phi_{\beta, \alpha} ; V_{\alpha}, \eta_{\beta, \alpha} ; \pi_{\alpha}\right\}$ where each $J_{\alpha}$ is a (finite dimensional real) Lie group, say with real Lie algebra $\mathfrak{j}_{\alpha}$, and if $\alpha \leqq \beta$ then $\phi_{\beta, \alpha}$ : $J_{\alpha} \rightarrow J_{\beta}$ is an analytic homomorphism. Let each of the $V_{\alpha}$ be a topological vector space, and assume that $\eta_{\beta, \alpha}: V_{\alpha} \rightarrow V_{\beta}$ is a continuous linear transformation for all $\alpha \leqq \beta$. Suppose further that $\pi_{\alpha}$ is a continuous representation of $J_{\alpha}$ on $V_{\alpha}$, and one has the obvious consistency condition that for $\alpha \leqq \beta$ the left hand diagram of

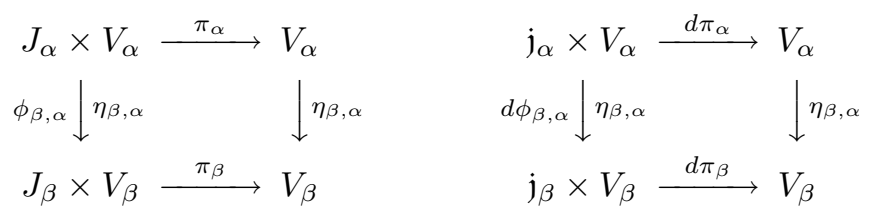

is commutative. If $\alpha \leqq \beta$ then $\phi_{\beta, \alpha}$ defines a Lie algebra homomorphism $d \phi_{\beta, \alpha}$ : $\mathfrak{j}_{\alpha} \rightarrow \mathfrak{j}_{\beta}$. The Lie algebra representations $d \pi_{\alpha}$ satisfy the consistency condition that comes out of the condition for the $\pi_{\alpha}$. So the right hand diagram above is commutative.

Let $J=\underline{\lim } J_{\alpha}, \mathfrak{j}=\lim _{\underline{j}} \mathfrak{\alpha}_{\alpha}$ and $V=\underline{\lim } V_{\alpha}$ in appropriate categories. The direct limit representation $\pi=\underline{\varliminf} \pi_{\alpha}$ is the representation of $J$ on $V$ given by $\pi\left(\left[g_{\alpha}\right]\right)\left(\left[v_{\alpha}\right]\right)=\left[\pi_{\alpha}\left(g_{\alpha}\right)\left(v_{\alpha}\right)\right]$. Similarly the direct limit representation $d \pi=\varliminf_{\longrightarrow} d \pi_{\alpha}$ is the representation of $\mathfrak{j}$ on $V$ given by $d \pi\left(\left[\xi_{\alpha}\right]\right)\left(\left[v_{\alpha}\right]\right)=\left[d \pi_{\alpha}\left(\xi_{\alpha}\right)\left(v_{\alpha}\right)\right]$. We then have the commutative diagrams

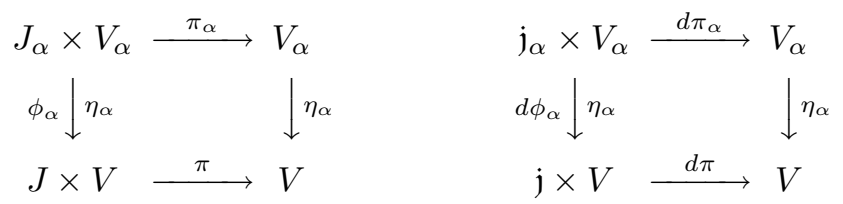


that were used in 24], 25] and [26] for the construction of differentiable structures on $J$. In the papers just cited, many considerations depended on growth conditions for a representation $\pi$ of a Lie group $J$. Those growth conditions are the imaginary spectral growth condition

$$
\text { if } \xi \in \mathfrak{j} \text { then } \sup \{|\operatorname{Im} \mu|: \mu \text { is in the spectrum of } d \pi(\xi)\}<\infty
$$

and the operator norm growth condition

$$
\text { if } \xi_{\alpha} \in \mathfrak{j}_{\alpha} \text { then } \sup _{\beta \geqq \alpha}\left\|d \pi_{\beta}\left(d \phi_{\beta, \alpha}\left(\xi_{\alpha}\right)\right)\right\|_{\mathrm{op}, \beta}<\infty .
$$

In (3.4a), $\operatorname{Im} \mu$ denotes the imaginary part of $\mu$. In (3.4b), $\alpha$ ranges over the index set, the $\pi_{\alpha}$ are assumed to be bounded 3 Banach representations, and $\|\cdot\|_{\mathrm{op}, \beta}$ refers to the operator norm on $V_{\beta}$. In our use of this condition, $J$ will usually be $G_{u}$ and $\pi$ will usually be a unitary representation $\pi_{u}$.

3.5. Lemma. Let $(\pi, H)=\underline{\lim }\left(\pi_{\alpha}, H_{\alpha}\right)$ be a unitary direct limit representation of a real Lie group $J=\underline{\lim } \overrightarrow{J_{\alpha}}$. Then the imaginary spectral growth condition (3.4a) and the operator norm growth condition (3.4b) are equivalent for $\left\{\pi_{\alpha}\right\}$. More precisely, $\pi$ satisfies the imaginary spectral growth condition (3.4a) if and only if $d \pi$ is a bounded representation of the Lie algebra $\mathfrak{j}$.

Proof. Since $\pi$ is unitary, every $d \pi(\xi), \xi \in \mathfrak{j}$, is essentially skew adjoint with pure imaginary spectrum.

3.6. Proposition. Let $\left\{H_{\alpha}, \eta_{\beta, \alpha}\right\}$ be a strict direct system of Hilbert spaces with unitary injections $\eta_{\beta, \alpha}$. Let $\left\{\pi_{u, \alpha}, H_{\alpha}\right\}$ be a strict direct system of unitary representations of a strict direct system $\left\{J_{u, \alpha}, \phi_{\beta, \alpha}\right\}_{\alpha, \beta \in A}$ of real compact Lie groups. Suppose that $\pi_{u}=\varliminf_{\longrightarrow} \pi_{u, \alpha}$ satisfies the growth conditions (3.4). Let $J_{\alpha}$ be a complexification of $J_{u, \alpha}$, in other words $J_{\alpha}$ is a complex Lie group whose Lie algebra is the complexification $\mathfrak{j}_{\alpha}$ of $\mathfrak{j}_{u, \alpha}$ where $J_{u, \alpha} \subset J_{\alpha}$ and $J_{u, \alpha}$ meets every topological component of $J_{\alpha}$. Then $J=\varliminf_{\longrightarrow} J_{\alpha}$ exists in the category of complex Lie groups, and $J$ is the complexification of $J_{u}=\lim _{u, \alpha}$.

Let $\pi_{\alpha}$ denote the holomorphic extension of $\pi_{u, \alpha}$ from $J_{u, \alpha}$ to $J_{\alpha}$. Then the representation $\pi_{u}=\underline{\lim } \pi_{u, \alpha}$ extends to a bounded representation $\pi$ of $J$, and $\pi=$ $\stackrel{\lim }{\longrightarrow} \pi_{\alpha}$. Further, if each $\pi_{\alpha}$ is (strongly) continuous and holomorphic then so is $\pi$.

Proof. Each $\mathfrak{j}_{\alpha}=\mathfrak{j}_{u, \alpha} \oplus \sqrt{-1} \mathfrak{j}_{u, \alpha}$. It follows that $\mathfrak{j}=\lim _{\mathbf{j}} \mathfrak{j}_{\alpha}=\mathfrak{j}_{u} \oplus \sqrt{-1} \mathfrak{j}_{u}$. The group $J_{\alpha}$ has polar decomposition $J_{\alpha}=J_{u, \alpha} \cdot \exp \left(\sqrt{-1} j_{u, \alpha}\right)$ and so the maps $\phi_{\beta, \alpha}$ extend to complex analytic group homomorphisms from $J_{\alpha}$ to $J_{\beta}$. The complexification $J$ of $J_{u}$ is then given by $J=J_{u} \cdot \exp \left(\sqrt{-1} \mathrm{j}_{u}\right)$.

We now have a direct system $\left\{J_{\alpha}, \phi_{\beta, \alpha}\right\}$. The complexified group $J$ satisfies the universal mapping condition A.3. Thus $J=\lim J_{\alpha}$ in the category of complex Lie groups.

As in the proof of Proposition B.1 of Appendix B, $V=\lim H_{\alpha}$ has an inner product by which it is realized as a pre-Hilbert space. Let $H$ be the completion of $V$. We now show that $\pi_{u}$ extends to a bounded representation $\pi$ of $J$ on $H$. In view of Lemma 3.5, $d \pi_{u}\left(\mathfrak{j}_{u}\right)$ consists of bounded operators, so $d \pi_{u}$ extends by linearity to a representation $d \pi$ of $\mathfrak{j}=\mathfrak{j}_{u} \oplus \sqrt{-1} \mathbf{j}_{u}$ by bounded linear operators. If $\xi \in \sqrt{-1} \mathbf{j}_{u}$ now

\footnotetext{
${ }^{3}$ If $T$ is a bounded operator on a Banach space then $\|T\|_{\text {op }}$ denotes its operator $\left(L_{\infty}\right)$ norm. A Banach space representation of a group or algebra is bounded if its image consists of bounded operators.
} 
$\exp (d \pi(\xi))$ is a well-defined bounded operator on $V$ and hence on the completion $H$ of $V$. Now using this and the polar decomposition $J=J_{u} \cdot \exp \left(\sqrt{-1} \mathrm{j}_{u}\right)$ we can extend $\pi_{u}$ to a map $\pi$ from $J$ to the bounded operators on $H$. By construction, $\pi(\exp (\xi))=\exp (d \pi(\xi))$ for every $\xi \in \sqrt{-1} \mathbf{j}_{u}$, and it follows that $\pi=\lim _{\alpha} \pi_{\alpha}$ is a group homomorphism.

Now assume that each $\pi_{\alpha}$ is (strongly) continuous. We will show that $\pi=\lim _{\longrightarrow} \pi_{\alpha}$ is (strongly) continuous. For this we have to show that the map $J \times V \rightarrow V$, given by $(g, v) \mapsto \pi(g) v$, is continuous. Since $J$ has the direct limit topology it suffices to show that each of the corresponding maps $J_{\alpha} \times V \rightarrow V$ is continuous. We first note that if $v \in V$ then the map $J_{\alpha} \rightarrow V$, by $g \mapsto \pi\left(\phi_{\alpha}(g)\right) v$ is continuous. This follows from the continuity of $\pi_{\alpha}$ for any $\alpha \geqq \alpha_{0}$ with $\eta_{\alpha_{0}}^{-1}(v)$ non-empty. Now for $g, g^{\prime} \in J_{\alpha}$ and $v, v^{\prime} \in V,\left\|\pi\left(\phi_{\alpha}(g)\right) v-\pi\left(\phi_{\alpha}\left(g^{\prime}\right)\right) v^{\prime}\right\| \leqq\left\|\pi\left(\phi_{\alpha}(g)\right)\left(v-v^{\prime}\right)\right\|+$ $\left\|\pi\left(\phi_{\alpha}(g)\right) v^{\prime}-\pi\left(\phi_{\alpha}\left(g^{\prime}\right)\right) v^{\prime}\right\|$. Each $\pi\left(\phi_{\alpha}(g)\right)$ is a bounded operator on $V$ and hence extends to the completion $H$. Since $g \mapsto \pi\left(\phi_{\alpha}(g)\right) v$ is continuous from $J_{\alpha}$ to $V$ and $J_{\alpha}$ is locally compact it follows from the Banach-Steinhaus Theorem that $\left\|\pi\left(\phi_{\alpha}(g)\right)\right\|$ is uniformly bounded on compact neighborhoods in $J_{\alpha}$. The continuity of $\pi$ follows.

By construction each $\pi_{\alpha}$ is holomorphic. For $v \in H$ define $f: J \rightarrow H$ by $f(g)=\pi(g) v$. We will show that $f$ is holomorphic. Since $J$ has the direct limit holomorphic structure, it is sufficient to show that $f \cdot \phi_{\alpha}$ is holomorphic for $\alpha$ sufficiently large. Choose a sequence $v_{n} \in V$ converging to $v$. Define $f_{n}: J \mapsto H$ by $f_{n}(g)=\pi(g) v_{n}$. Let $C \subset J_{\alpha}$ be compact. Continuity of $\pi$ and the BanachSteinhaus Theorem imply that the family of operators $\pi\left(\phi_{\alpha}(g)\right), g \in C$, is uniformly bounded. Now $\left\{f_{n} \cdot \phi_{\alpha}\right\} \rightarrow f \cdot \phi_{\alpha}$ uniformly on $C$. As the $\pi_{\alpha}$ are holomorphic, the $f_{n} \cdot \phi_{\alpha}$ are holomorphic and it follows that $f \cdot \phi_{\alpha}$ is holomorphic. Now $\pi: J \times H \rightarrow H$ is continuous and separately holomorphic, so it is holomorphic. That completes the proof.

Let $\tau=\lim \tau_{\alpha}$ be the direct limit representation of $L=\lim L_{\alpha}$ on the preHilbert space $E=\lim E_{\alpha}$. Assume the operator norm growth condition for $\tau$, so $\tau(g)$ is a bounded operator for each $g \in L$. Further by 3.2 it has lowest weight $-\lambda$. As usual we extend $\tau$ to $Q$ by $\tau(\ell u)=\tau(\ell)$ for $\ell \in L$ and $u \in Q$. The associated $G^{-}$

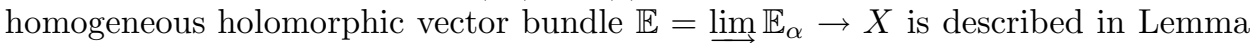
1.4. The dual representation $\tau^{*}=\varliminf \tau_{\alpha}^{*}$ of $L$ on $E^{*}=\varliminf_{\alpha} E_{\alpha}^{*}$ has highest weight $\lambda$, and the associated $G$-homogeneous holomorphic vector bundle is the dual bundle $\mathbb{E}^{*} \rightarrow X$. See Appendix B for a discussion of inverse limit representations. We want to examine the

$$
\pi_{\alpha}^{q}:=\text { natural action of } G_{\alpha} \text { on } H^{q}\left(X_{\alpha} ; \mathcal{O}\left(\mathbb{E}_{\alpha}^{*}\right)\right)
$$

The classical Borel-Weil Theorem tells us that each $\pi_{\alpha}=\pi_{\alpha}^{0}$ is an irreducible finite dimensional holomorphic representation with a unique (up to positive real factor) $\pi_{\alpha}\left(G_{u, \alpha}\right)$-invariant hermitian inner product.

3.8. Proposition. Suppose that each $\lambda_{\alpha}$ is integral and $\Delta^{+}\left(\mathfrak{g}_{\alpha}, \mathfrak{h}_{\alpha}\right)$-dominant. Then each

$$
\zeta_{\beta, \alpha}: H^{0}\left(X_{\beta} ; \mathcal{O}\left(\mathbb{E}_{\beta}^{*}\right)\right) \rightarrow H^{0}\left(X_{\alpha} ; \mathcal{O}\left(\mathbb{E}_{\alpha}^{*}\right)\right)
$$


is surjective. Consider inverse limits in the Hilbert category,

$$
\begin{aligned}
\pi=\varliminf_{\alpha}:= & \text { inverse limit action of } G \\
& \text { on } H^{0}\left(X ; \mathcal{O}\left(\mathbb{E}^{*}\right)\right)=\varliminf_{\pi_{u}}^{\lim }\left\{H^{0}\left(X_{\alpha} ; \mathcal{O}\left(\mathbb{E}_{\alpha}^{*}\right)\right), \zeta_{\alpha}\right\}, \\
\pi_{u, \alpha}:= & \text { inverse limit action of } G_{u} \\
& \text { on } H^{0}\left(X ; \mathcal{O}\left(\mathbb{E}^{*}\right)\right), \text { so } \pi_{u}=\left.\pi\right|_{G_{u}} .
\end{aligned}
$$

Then the following hold.

(i) $H^{0}\left(X ; \mathcal{O}\left(\mathbb{E}^{*}\right)\right)$ has a unique (up to positive real factor) $\pi\left(G_{u}\right)$-invariant Hilbert space structure, and $\pi_{u}$ is a unitary representation of $G_{u}$ on $H^{0}\left(X ; \mathcal{O}\left(\mathbb{E}^{*}\right)\right)$.

(ii) If $\pi_{u}$ satisfies the imaginary spectral growth condition, then $\pi$ is a bounded, strongly continuous, holomorphic representation of $G$.

Proof. The normalizations (0.1), (0.2) and (0.3) show that $\zeta_{\beta, \alpha}$ carries a nonzero highest weight vector $w_{\beta}$ for $\pi_{\beta}$ to a nonzero highest weight vector $w_{\alpha}$ for $\pi_{\alpha}$. So

$$
\zeta_{\beta, \alpha}\left(d \pi_{\beta}\left(d \phi_{\beta, \alpha}(\Xi)\right)\left(w_{\beta}\right)\right)=d \pi_{\alpha}(\Xi)\left(w_{\alpha}\right) \text { for all } \Xi \in \mathcal{U}\left(\mathfrak{g}_{\alpha}\right),
$$

essentially as in (3.2). Surjectivity of $\zeta_{\beta, \alpha}$ follows. (This proof also shows that the projection $\zeta_{\alpha}$ from $H^{0}\left(X ; \mathcal{O}\left(\mathbb{E}^{*}\right)\right)$ to $H^{0}\left(X_{\alpha} ; \mathcal{O}\left(\mathbb{E}_{\alpha}^{*}\right)\right)$ is surjective.)

The classical Borel-Weil Theorem says that $\pi_{\alpha}$ is the irreducible representation of $G_{\alpha}$ of highest weight $\lambda_{\alpha}$. The restrictions $\pi_{u, \alpha}=\left.\pi_{\alpha}\right|_{G_{u, \alpha}}$ are unitary representations of the $G_{u, \alpha}$. By (3.10) the $\pi_{u, \alpha}$ form an inverse system and so we have a representation $\pi_{u}=\varliminf^{\lim } \pi_{u, \alpha}$ of $G_{u}$ on $H^{0}\left(X ; \mathcal{O}\left(\mathbb{E}^{*}\right)\right)$. Now, as in Lemma 3.3 we may suppose that each $\zeta_{\beta, \alpha}^{*}: H^{0}\left(X_{\alpha} ; \mathcal{O}\left(\mathbb{E}_{\alpha}^{*}\right)\right)^{*} \rightarrow H^{0}\left(X_{\beta} ; \mathcal{O}\left(\mathbb{E}_{\beta}^{*}\right)\right)^{*}$ is a unitary injection. That gives the $\pi\left(G_{u}\right)$-invariant pre-Hilbert space structure on $\varliminf_{\mathrm{B})} H^{0}\left(X_{\alpha} ; \mathcal{O}\left(\mathbb{E}_{\alpha}^{*}\right)\right)^{*}$. Using the duality described in Proposition B.2 (see Appendix B) we have that

$$
H^{0}\left(X ; \mathcal{O}\left(\mathbb{E}^{*}\right)\right)=\lim H^{0}\left(X_{\alpha} ; \mathcal{O}\left(\mathbb{E}_{\alpha}^{*}\right)\right)=\left(\underline{\lim _{1}} H^{0}\left(X_{\alpha} ; \mathcal{O}\left(\mathbb{E}_{\alpha}^{*}\right)\right)^{*}\right)^{*}
$$

has a Hilbert space structure and $\pi_{u}$ is a unitary representation of $G_{u}$.

The imaginary spectral growth condition for $\pi_{u}$ is equivalent to the operator norm growth condition (3.4b) because $\pi_{u}$ is unitary. This shows that $\pi$ is bounded, or one can argue as follows. By Theorem B.10, the system $\left\{\pi_{u, \alpha}^{*}, H^{0}\left(X_{\alpha} ; \mathcal{O}\left(\mathbb{E}_{\alpha}^{*}\right)\right)^{*}\right\}$ forms a direct system of representations which gives the dual representation $\pi_{u}^{*}=$ $\longleftrightarrow \pi_{u, \alpha}^{*}$ of $G_{u}$. Now if $\pi_{u}$ satisfies the imaginary spectral growth condition (3.4a), so does $\pi_{u}^{*}$. It then follows from Proposition 3.6 that $\pi_{u}^{*}$ extends to a bounded representation $\pi^{*}$ of $G$ on $H^{0}\left(X ; \mathcal{O}\left(\mathbb{E}^{*}\right)\right)^{*}$ and so $\pi=\lim _{\alpha}$ is a bounded representation of $G$ on $H^{0}\left(X ; \mathcal{O}\left(\mathbb{E}^{*}\right)\right)$.

It remains to be shown that $\pi$ is (strongly) continuous and holomorphic. We first verify the continuity. Set $H=H^{0}\left(X ; \mathcal{O}\left(\mathbb{E}^{*}\right)\right)$ and $V_{\alpha}=H^{0}\left(X_{\alpha} ; \mathcal{O}\left(\mathbb{E}_{\alpha}^{*}\right)\right)$. We have to show that the map $G \times H \rightarrow H$, given by $(g, h) \mapsto \pi(g) h$, is continuous. We already know that $G$ acts on $H$ by bounded operators. Since $G$ has the direct limit topology it is enough to show the corresponding map $\mu_{\alpha}: G_{\alpha} \times H \rightarrow H$ is continuous for every $\alpha$ where $\mu_{\alpha}(g, h)=\pi\left(\phi_{\alpha}(g)\right) h$. Continuity of $\pi_{\alpha}$ implies 
continuity for the corresponding maps $\nu_{\alpha}: G_{\alpha} \times V_{\alpha} \rightarrow V_{\alpha}$. Further, the diagram

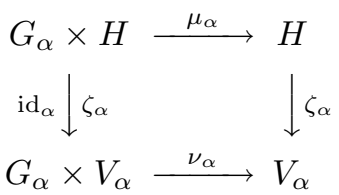

commutes. Here id $\mathrm{d}_{\alpha}$ is the identity function on $G_{\alpha}$. Continuity of $\nu_{\alpha}$ and $\zeta_{\alpha}$ implies continuity of $\nu_{\alpha} \cdot\left(\operatorname{id}_{\alpha} \times \zeta_{\alpha}\right)=\zeta_{\alpha} \cdot \mu_{\alpha}$. But $\zeta_{\alpha}$ is surjective, so the Open Mapping Theorem says that $\zeta_{\alpha}$ is open. We have proved that $\mu_{\alpha}$ is continuous.

Propositions 3.6 and B.10 now show that $\pi$ is a holomorphic representation.

Fix the Hilbert structure on $H^{0}\left(X ; \mathcal{O}\left(\mathbb{E}^{*}\right)\right)$ given by (3.11). Then from Proposition 3.8 we have the direct limit Borel-Weil Theorem as follows.

3.13. Theorem. The natural action $\pi$ of $G$ on $H^{0}\left(X ; \mathcal{O}\left(\mathbb{E}^{*}\right)\right)$ is an irreducible representation of highest weight $\lambda$, and $\pi_{u}=\left.\pi\right|_{G_{u}}$ is an irreducible unitary representation of $G_{u}$ on $H^{0}\left(X ; \mathcal{O}\left(\mathbb{E}^{*}\right)\right)$ that also has highest weight $\lambda$. If $\pi_{u}$ satisfies the growth conditions (3.4) then the representation $\pi$ is bounded, strongly continuous, and holomorphic.

Proof. We only need to check that $\pi$ is irreducible. Let $H=H^{0}\left(X ; \mathcal{O}\left(\mathbb{E}^{*}\right)\right)$ and $H_{\alpha}=H^{0}\left(X_{\alpha} ; \mathcal{O}\left(\mathbb{E}_{\alpha}^{*}\right)\right)$. Let $V$ be a non-zero, closed, $G$-invariant subspace of $H$. Then $\zeta_{\alpha}(V)$ is a closed (since $H_{\alpha}$ is finite dimensional), $G_{\alpha}$-invariant subspace of $H_{\alpha}$ for every $\alpha$. Since $V \neq 0$ there is an index $\alpha_{0} \in A$ such that $\zeta_{\alpha}(V) \neq 0$ whenever $\alpha \geqq \alpha_{0}$. Irreducibility of $\pi_{\alpha}$ now implies $\zeta_{\alpha}(V)=H_{\alpha}$ for all such $\alpha$. The universal property of inverse limits now implies $V=H$, so $\pi$ is irreducible.

Since the imaginary spectral growth condition is essential for parts of our BorelWeil and Bott-Borel-Weil Theorems, we characterize the highest weights $\lambda$ that correspond to imaginary spectral growth representations of $G_{u}$. As usual, a weight $\lambda \in \mathfrak{h}^{*}$ is said to be dominant integral if $d \phi_{\beta}^{*}(\lambda) \in \mathfrak{h}_{\beta}^{*}$ is dominant integral for every sufficiently large index $\beta$.

3.14. Proposition. Let $\lambda \in \mathfrak{h}^{*}$ be a dominant integral weight. Let $\alpha_{0}$ be an index such that $\lambda_{\beta}=d \phi_{\beta}^{*}(\lambda)$ is dominant integral for all $\beta \geqq \alpha_{0}$ in $A$. For $\beta \geqq \alpha_{0}$ let $\left(\pi_{u, \beta}, V_{\beta}\right)$ be the irreducible representation of $G_{u, \beta}$ with highest weight $\lambda_{\beta}$. Let $\pi_{u}=$ $\stackrel{\varliminf}{\longrightarrow} \pi_{u, \beta}$, let $V=\varliminf_{\beta} V_{\beta}$ equipped with its pre-Hilbert space structure, and let $\widetilde{V}$ be

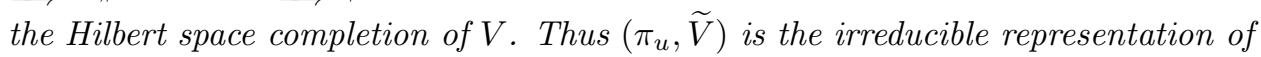
$G_{u}$ with highest weight $\lambda$. The following are equivalent:

(i) $\pi_{u}$ satisfies the imaginary spectral growth condition (3.4a),

(ii) $\pi_{u}$ satisfies the operator norm growth condition (3.4b),

(iii) $m_{\lambda}(h):=\lim \sup _{\beta} \geqq \alpha_{0}\left(\max _{w_{\beta} \in W_{\beta}}\left\{\left|\lambda_{\beta}\left(w_{\beta} h_{\beta}\right)\right|\right\}\right)<\infty$ for every $h$ in $\mathfrak{h}_{u}$,

(iv) For every $\xi$ in the complex Lie algebra $\mathfrak{g}, d \pi(\xi)$ is a bounded operator on $V$.

Proof. $(i) \Leftrightarrow(i i)$ is Lemma 3.5. And $(i v) \Rightarrow(i i)$ is obvious. 
$(i) \Rightarrow(i i i)$. If $\xi \in \mathfrak{g}_{u}$ the operator $d \pi_{u}(\xi)$ is skew adjoint so its spectrum is purely imaginary. Hence for $h$ in $\mathfrak{h}_{u}$ we have

$$
\begin{aligned}
\iota(h) & =\limsup _{\beta \geqq \alpha_{0}}\left(\max \left\{|\operatorname{Im} \mu| \mid \mu \text { is an eigenvalue of } d \pi_{u, \beta}\left(h_{\beta}\right)\right\}\right) \\
& =\limsup _{\beta \geqq \alpha_{0}}\left(\max \left\{|\mu| \mid \mu \text { is an eigenvalue of } d \pi_{u, \beta}\left(h_{\beta}\right)\right\}\right) \\
& =\limsup _{\beta \geqq \alpha_{0}}\left(\max \left\{\left|\mu\left(d \pi_{u, \beta} h_{\beta}\right)\right| \text { where } \mu \text { is a weight of } d \pi_{u, \beta}\right\}\right) \\
& =\limsup _{\beta \geqq \alpha_{0}}\left(\max _{w_{\beta} \in W_{\beta}}\left\{\left|\lambda_{\beta}\left(w_{\beta} h_{\beta}\right)\right|\right\}\right)=m_{\lambda}(h) .
\end{aligned}
$$

In the last line we used the fact that the weights of $d \pi_{u, \beta}$ are in the convex hull of the weights of the orbit of $\lambda_{\beta}$ under the Weyl group $W_{\beta}$. Thus if $\pi_{u}$ is spectral growth, then $m_{\lambda}(h)$ is finite for every $h$ in $\mathfrak{h}_{u}$.

$($ iii $) \Rightarrow($ iv $)$. Assume that $m_{\lambda}(h)<\infty$ for each $h \in \mathfrak{h}_{u}$. To prove that every $d \pi(\xi)$ is bounded, we write $\xi$ in terms of the triangular decomposition of $\mathfrak{g}, \xi=y+h+x$, with $y \in \mathfrak{n}^{-}, h \in \mathfrak{h}$ and $x \in \mathfrak{n}^{+}$. It is enough to show that each of the operators $d \pi(y), d \pi(h)$ and $d \pi(x)$ is bounded.

For $h \in \mathfrak{h}$ write $h=h_{1}+i h_{2}$ where $h_{1}$ and $h_{2}$ are in the real Cartan subalgebra $\mathfrak{h}_{u}$. Then $\pi(h)=d \pi_{u}\left(h_{1}\right)+i d \pi_{u}\left(h_{2}\right)$ so the operator norm $\|d \pi(h)\| \leqq m_{\lambda}(h) \leqq$ $m_{\lambda}\left(h_{1}\right)+m_{\lambda}\left(h_{2}\right)<\infty$.

For $y$ in $\mathfrak{n}^{-}$, choose $h \in \mathfrak{h}$ and $x \in \mathfrak{n}^{+}$such that $[h, y]=-2 y,[x, y]=h$ and $[h, x]=2 x$. Let $\mathfrak{s} \cong \mathfrak{s l}(2, \mathbb{C})$ denote the subalgebra of $\mathfrak{g}$ spanned by $y, h$ and $x$. Let $v \in V$ and let $\alpha \geqq \alpha_{0}$ large enough so that there exist (i) elements $y_{\alpha}, h_{\alpha}, x_{\alpha} \in \mathfrak{g}_{\alpha}$ with $d \phi_{\alpha}\left(y_{\alpha}\right)=y, d \phi_{\alpha}\left(h_{\alpha}\right)=h$ and $d \phi_{\alpha}\left(x_{\alpha}\right)=x$, and (ii) an element $v_{\alpha} \in V_{\alpha}$ such that $\eta_{\alpha}\left(v_{\alpha}\right)=v$. Here $\eta_{\alpha}: V_{\alpha} \rightarrow V$ is the canonical homomorphism. The $\mathfrak{s}$-submodule $U \subset V$, generated by $v$, is irreducible for $\mathfrak{s}$ and contained in $\eta_{\alpha}\left(V_{\alpha}\right)$. As $V_{\alpha}$ is finite dimensional now $U$ is an irreducible highest weight $\mathfrak{s}$-module. The highest weight is $k \leqq\left|\lambda_{\alpha}\left(h_{\alpha}\right)\right| \leqq m_{\lambda}(h)$.

Now $V=\bigoplus V_{k}$ where $V_{k}$ is a (possibly infinite) multiple of the irreducible $\mathfrak{s}^{-}$ module of highest weight $k \in \mathbb{Z}^{+}$. Each $k$, for which $V_{k} \neq 0$, is less than or equal to $m_{\lambda}(h)$. The operator norm of $d \pi(y)$ on $V$ is the maximum of the operator norms of $d \pi(y)$ on $V_{k}$. Here each $k \leqq m_{\lambda}(h)$, so $\|d \pi(y)\|<\infty$. The same argument shows $\|d \pi(x)\|<\infty$ for $x \in \mathfrak{n}^{+}$. That completes the proof.

We thank Roe Goodman for the idea of the argument of $(i i i) \Rightarrow(i v)$.

Even when $\lambda \in \mathfrak{h}^{*}$ fails to be dominant integral, the proof of (3.14) shows that the spectral growth condition for $\pi_{u}$ implies $m_{\lambda}(h)<\infty$ for all $h \in \mathfrak{h}_{u}$.

\section{The Bott-Borel-Weil Theorem}

In this section we indicate an extension of the Borel-Weil Theorem 3.13 to higher cohomology. The main consideration is to keep cohomology in a fixed degree so that a good analogue of Proposition 3.8 can be formulated. Several cases are known ([20], [15]) where this is possible for direct limits of noncompact semisimple Lie groups. Here we work out conditions under which cohomology degrees stabilize for direct limits of compact Lie groups. Our Bott-Borel-Weil Theorem 4.6 is proved under those conditions. 
Let $\tau_{\alpha}$ have lowest weight $-\lambda_{\alpha}$ as in (3.1). Write $\rho_{\alpha}$ for half the sum of the elements of $\Delta_{\alpha}^{+}=\Delta^{+}\left(\mathfrak{g}_{\alpha}, \mathfrak{h}_{\alpha}\right)$ and denote

$$
q_{\alpha}=q_{\alpha}\left(\lambda_{\alpha}+\rho_{\alpha}\right)=\left|\left\{\nu_{\alpha} \in \Delta_{\alpha}^{+} \mid\left\langle\lambda_{\alpha}+\rho_{\alpha}, \nu_{\alpha}\right\rangle<0\right\}\right|,
$$

as usual. If $\lambda_{\alpha}+\rho_{\alpha}$ is singular, i.e. if $\left\langle\lambda_{\alpha}+\rho_{\alpha}, \nu_{\alpha}\right\rangle=0$ for some $\nu_{\alpha} \in \Delta_{\alpha}^{+}$, then the classical Bott-Borel-Weil Theorem says that every cohomology $H^{q}\left(X_{\alpha} ; \mathcal{O}\left(\mathbb{E}_{\alpha}^{*}\right)\right)=$ 0 . If $\lambda_{\alpha}+\rho_{\alpha}$ is nonsingular we define

$$
\begin{aligned}
w_{\alpha} \in W\left(\mathfrak{g}_{\alpha}, \mathfrak{h}_{\alpha}\right) \\
:=\text { Weyl group element that carries } \lambda_{\alpha}+\rho_{\alpha} \text { to a dominant weight, } \\
\quad \text { in other words such that }\left\langle w_{\alpha}\left(\lambda_{\alpha}+\rho_{\alpha}\right), \nu_{\alpha}\right\rangle>0 \text { for all } \nu_{\alpha} \in \Delta_{\alpha}^{+} .
\end{aligned}
$$

Then $w_{\alpha}$ has length $\ell\left(w_{\alpha}\right)=q_{\alpha}$ in a minimal length expression as a product of simple root reflections. The classical Bott-Borel-Weil Theorem says that

$$
\begin{aligned}
& H^{q}\left(X_{\alpha} ; \mathcal{O}\left(\mathbb{E}_{\alpha}^{*}\right)\right)=0 \text { for } q \neq q_{\alpha}, \\
& G_{\alpha} \text { acts irreducibly on } H^{q_{\alpha}}\left(X_{\alpha} ; \mathcal{O}\left(\mathbb{E}_{\alpha}^{*}\right)\right) \\
& \text { with highest weight } w_{\alpha}\left(\lambda_{\alpha}+\rho_{\alpha}\right)-\rho_{\alpha} .
\end{aligned}
$$

So our problem here is to find situations in which there exists an index $\alpha_{0}$ such that $q_{\alpha}$ is constant for $\alpha \geqq \alpha_{0}$. (In the case of the Borel-Weil Theorem this occurs with all $q_{\alpha}=0$.) In order to do that we need a condition on the weight $\lambda$ of (3.1).

We fix $\mathfrak{b}$, the Borel subalgebra $\lim _{\longrightarrow} \mathfrak{b}_{\alpha}$ of $\mathfrak{g}$ defined by $(0.1),(0.2)$ and $(0.3)$ with $\mathfrak{b}_{\alpha} \subset \mathfrak{q}_{\alpha}$ for all $\alpha$. Definitions 4.4 and 4.5 below depend on this choice of Borel subalgebra $\mathfrak{b} \subset \mathfrak{g}$.

4.4. Definitions. The Weyl group $W=W(\mathfrak{g}, \mathfrak{h})$ is the group of all $\left.w\right|_{\mathfrak{h}}$ where $w$ is an automorphism of $\mathfrak{g}$ as follows. First, $w(\mathfrak{h})=\mathfrak{h}$. Second, there is an index $\alpha_{0}=\alpha_{0}(w)$ such that: if $\beta \geqq \alpha_{0}$, then $w\left(d \phi_{\beta}\left(\mathfrak{g}_{\beta}\right)\right)=d \phi_{\beta}\left(\mathfrak{g}_{\beta}\right)$ and $\left.w\right|_{d \phi_{\beta}\left(\mathfrak{g}_{\beta}\right)}$ is an inner automorphism of $d \phi_{\beta}\left(\mathfrak{g}_{\beta}\right)$. The finite Weyl group is $W_{F}=W_{F}(\mathfrak{g}, \mathfrak{b}, \mathfrak{h})=$ $\{w \in W \mid w(\mathfrak{b}) \cap \mathfrak{b}$ has finite codimension in $\mathfrak{b}\}$. If $w \in W_{F}$ then $w(\mathfrak{b}) \cap \mathfrak{b}$ has the same codimension in $\mathfrak{b}$ and in $w(\mathfrak{b})$, and we define this codimension to be the length $\ell(w)$.

Remark. There are many other possible choices for the definition of the Weyl group. For example one has the smaller classical Weyl group $W_{c l}=W_{c l}(\mathfrak{g}, \mathfrak{h})$ given by the quotient $N_{G}(\mathfrak{h}) / Z_{G}(\mathfrak{h})$ of the normalizer of a Cartan subalgebra modulo the centralizer, and one has a certain much larger total Weyl group $W_{\text {tot }}=W_{\text {tot }}(\mathfrak{g}, \mathfrak{h})$. Although $W_{c l}(\mathfrak{g}, \mathfrak{h}) \varsubsetneqq W(\mathfrak{g}, \mathfrak{h}) \varsubsetneqq W_{\text {tot }}(\mathfrak{g}, \mathfrak{h})$, these all lead to the same finite Weyl group. See [31] for a complete discussion. Our choice (4.4) of definition of Weyl group in this paper is not really so important, because we make serious use only of the finite Weyl group.

Remark. Whenever $\gamma \in A$, identify $\mathfrak{g}_{\gamma}$ and $\mathfrak{h}_{\gamma}$ with their $d \phi_{\gamma}$-images, and identify $\mathfrak{h}_{\gamma}$ with $\mathfrak{h}_{\gamma}^{*}$ by means of the Cartan-Killing form of $\mathfrak{g}_{\gamma}$. If the Cartan-Killing forms are aligned in the sense of (7.1b) below, and if the alignment constants satisfy (7.8), then $\mathfrak{g}$ has a Cartan-Killing form as in (7.9), and it intertwines the actions of $W$ on $\mathfrak{h}$ and on $\mathfrak{h}^{*}$. In any case, if $w \in W_{F}$ there is a cofinal subset $B$ of the index set $A$ such that: if $\beta \in B$ then, directly from the Definitions 4.4,

(i) $w\left(\Delta_{\beta}\right)=\Delta_{\beta}$ and $w\left(\Delta_{\alpha}\right)=\Delta_{\alpha}$,

(ii) $w_{\beta}=\left.w\right|_{\mathfrak{h}_{\beta}} \in W\left(\mathfrak{g}_{\beta}, \mathfrak{h}_{\beta}\right)$ and $w_{\alpha}=\left.w\right|_{\mathfrak{h}_{\alpha}} \in W\left(\mathfrak{g}_{\alpha}, \mathfrak{h}_{\alpha}\right)$, and 
(iii) the classically defined lengths $\ell\left(w_{\beta}\right)=\ell\left(w_{\alpha}\right)$ relative to $\Delta_{\beta}^{+}$and $\Delta_{\alpha}^{+}$, respectively.

As $\ell\left(w_{\alpha}\right)$ is non-decreasing in $\alpha$, there is an index $\alpha_{0}$ (which may depend on $w$ ) such that the $\ell(w)$ of Definition 4.4 is the common value of the classical lengths $\ell\left(w_{\beta}\right)$ for $\beta \geqq \alpha_{0}$.

The idea of grouping parabolics whose intersection has finite codimension in each, as in commensurablility for discrete subgroups of Lie groups, seems to be due to I. Dimitrov and I. Penkov.

4.5. Definitions. A linear functional $\lambda \in \mathfrak{h}^{*}$ is classically cohomologically finite if there exist $w \in W_{F}$ and $\alpha_{0}$ as above, and an integral (exponentiates to $H$ ) $\widetilde{\lambda} \in \mathfrak{h}^{*}$ with the following property: if $\beta \geqq \alpha_{0}(w)$ then $d \phi_{\beta}^{*}(\widetilde{\lambda})$ is dominant relative to $\Delta^{+}\left(\mathfrak{g}_{\beta}, \mathfrak{h}_{\beta}\right)$ and $d \phi_{\beta}^{*}(\widetilde{\lambda})=w_{\beta}\left(\lambda_{\beta}+\rho_{\beta}\right)-\rho_{\beta}$. A linear functional $\lambda \in \mathfrak{h}^{*}$ is dominant if it is classically cohomologically finite using the identity element of $W_{F}$, in other words with $\lambda=\widetilde{\lambda}$. A linear functional $\lambda \in \mathfrak{h}^{*}$ is cohomologically finite of degree $q_{\lambda}$ if, for $\alpha$ sufficiently large, $\lambda_{\alpha}+\rho_{\alpha}$ is nonsingular and $q_{\lambda}=q_{\alpha}\left(\lambda_{\alpha}+\rho_{\alpha}\right)$ as defined in (4.1).

If $\lambda \in \mathfrak{h}^{*}$ is classically cohomologically finite and $\beta$ is sufficiently large, as above, then $d \phi_{\beta}^{*}(\widetilde{\lambda})+\rho_{\beta}=w_{\beta}\left(\lambda_{\beta}+\rho_{\beta}\right)$ is $\mathfrak{g}_{\beta}$-nonsingular, so $\lambda_{\beta}+\rho_{\beta}$ also is $\mathfrak{g}_{\beta}$-nonsingular. Thus, if $\lambda$ is classically cohomologically finite by means of $w \in W_{F}$, then it is cohomologically finite of degree $q_{\lambda}=\ell(w)$.

Later we will see examples where $\lambda$ is cohomologically finite of degree $q>0$ while $W_{F}=\{1\}$ so that $\lambda$ cannot be classically cohomologically finite.

The Borel-Weil Theorem 3.13 applies precisely in the case where $\lambda$ is dominant.

We proceed by applying the idea of Section 3. Let $\lambda \in \mathfrak{h}^{*}$ be integral and cohomologically finite of degree $q_{\lambda}$. Define $\mathbb{E} \rightarrow X$ as in Lemma 1.4, let $\pi_{\alpha}^{q}$ denote the natural action of $G_{\alpha}$ on $H^{q}\left(X_{\alpha} ; \mathcal{O}\left(\mathbb{E}_{\alpha}^{*}\right)\right)$. Let $\pi^{q}=\lim \pi_{\alpha}^{q}$, natural inverse limit action of $G$ on $\left.H^{q}\left(X ; \mathcal{O}\left(\mathbb{E}^{*}\right)\right)=\lim _{\lfloor}\left\{H^{q}\left(X_{\alpha} ; \mathcal{O}\left(\mathbb{E}_{\alpha}^{*}\right)\right), \zeta_{\alpha}\right)\right\}$. As in Proposition 3.8 we have a $\pi^{q}\left(G_{u}\right)$-invariant Hilbert space structure on $H^{q}\left(X ; \mathcal{O}\left(\mathbb{E}^{*}\right)\right)$. Then we have a Bott-Borel-Weil Theorem as follows.

4.6. Theorem. Let $\lambda \in \mathfrak{h}^{*}$ be integral so that $\mathbb{E} \rightarrow X$ is well defined.

1. If $\lambda$ is not cohomologically finite then every $H^{q}\left(X ; \mathcal{O}\left(\mathbb{E}^{*}\right)\right)=0$.

2. Assume that $\lambda$ is cohomologically finite of degree $q_{\lambda}$. Then

(i) $H^{q}\left(X ; \mathcal{O}\left(\mathbb{E}^{*}\right)\right)=0$ for $q \neq q_{\lambda}$,

(ii) the inverse limit representation $\pi_{u}^{q_{\lambda}}$ of $G_{u}$ on $H^{q_{\lambda}}\left(X ; \mathcal{O}\left(\mathbb{E}^{*}\right)\right)$ is an irreducible unitary representation, and

(iii) if $\pi_{u}^{q_{\lambda}}$ satisfies the spectral growth condition (3.4) then $\pi_{u}^{q_{\lambda}}$ extends to a bounded, strongly continuous, holomorphic representation $\pi^{q_{\lambda}}$ of $G$ on $H^{q_{\lambda}}\left(X ; \mathcal{O}\left(\mathbb{E}^{*}\right)\right)$.

3. If, further, $\lambda$ is classically cohomologically finite, by means of $w \in W_{F}$, then $q_{\lambda}=\ell(w)$ and the inverse limit representation $\pi_{u}^{q_{\lambda}}$ of $G_{u}$ on $H^{q_{\lambda}}\left(X ; \mathcal{O}\left(\mathbb{E}^{*}\right)\right)$ has highest weight $\widetilde{\lambda}$ as in Definition 4.5 .

Proof. Suppose first that $\lambda$ is not cohomologically finite. Then the $q_{\alpha}\left(\lambda_{\alpha}+\rho_{\alpha}\right)$ are unbounded, for they are increasing in $\alpha$ and do not stabilize. Thus $H^{q}\left(X ; \mathcal{O}\left(\mathbb{E}^{*}\right)\right)$ $=\lim _{\longleftarrow}\left\{H^{q}\left(X_{\alpha} ; \mathcal{O}\left(\mathbb{E}_{\alpha}^{*}\right)\right), \zeta_{\alpha}\right\}=0$ for every $q \geqq 0$.

Now suppose that $\lambda$ is cohomologically finite of degree $q_{\lambda}$. Then $H^{q}\left(X ; \mathcal{O}\left(\mathbb{E}^{*}\right)\right)$ $=\varliminf_{\varlimsup}\left\{H^{q}\left(X_{\alpha} ; \mathcal{O}\left(\mathbb{E}_{\alpha}^{*}\right)\right), \zeta_{\alpha}\right\}=0$ for every $q \neq q_{\lambda}$. If we view the $H^{q_{\lambda}}\left(X_{\alpha} ; \mathcal{O}\left(\mathbb{E}_{\alpha}^{*}\right)\right)$ 
as Dolbeault cohomology spaces we see that the inverse system maps eventually are surjective, so $H^{q_{\lambda}}\left(X ; \mathcal{O}\left(\mathbb{E}^{*}\right)\right) \neq 0$, and the remaining assertions follow as in Proposition 3.8 .

Our question, now, is to pinpoint the conditions under which $\lambda$ is cohomologically finite or even classically cohomologically finite. In $\S 7$ below we give a general sufficient condition for classical cohomological finiteness. But in $\S \S 5$ and 6 , we give precise criteria on this for the very interesting "diagonal embedding", "standard embedding", and "Warhol embedding", direct systems.

\section{Diagonal Embedding Direct Limits}

We examine a variation on the diagonal embedding direct limits of [33] and [4]. The purpose of the variation is to ensure the validity of the basic requirements $(0.1)$, (0.2) and (0.3) for our Borel-Weil Theorem. We work out a precise criterion for classical cohomological finiteness in diagonal embedding direct systems, in order to apply Theorem 4.6 in the context of of diagonal embeddings. We also give examples of non-classical cohomological finiteness and we characterize the spectral growth condition.

If $A_{n}$ are matrices of degrees $d_{n}$ for $1 \leqq n \leqq r$ then $\operatorname{diag}\left\{A_{1}, \ldots, A_{r}\right\}$ denotes the matrix of degree $d=d_{1}+\cdots+d_{r}$ with block diagonal entries $A_{1}$ through $A_{r}$.

Type A. We look at diagonal embeddings of unitary groups. Fix sequences $\mathbf{r}=\left\{r_{n}\right\}_{n \geqq 0}, \mathbf{s}=\left\{s_{n}\right\}_{n \geqq 0}$ and $\mathbf{t}=\left\{t_{n}\right\}_{n \geqq 0}$ of non-negative integers with $r_{0}>0=$ $s_{0}=t_{0}$. Let $d_{0}=r_{0}, G_{u, 0}=S U\left(d_{0}\right)$, and $G_{0}=S L\left(d_{0} ; \mathbb{C}\right)$. Recursively define $G_{u, n+1}=S U\left(d_{n+1}\right)$ and $G_{n+1}=S L\left(d_{n+1} ; \mathbb{C}\right)$ where $d_{n+1}=d_{n}\left(r_{n+1}+s_{n+1}\right)+$ $t_{n+1}$. Write $A^{t}$ for the transpose of a matrix $A$. The outer automorphisms $\psi$ of $S L(d ; \mathbb{C})$ and $d \psi$ of $\mathfrak{s l}(d ; \mathbb{C})$ defined by

$$
\psi(g)=J\left(g^{t}\right)^{-1} J^{-1} \text { and } d \psi(\eta)=-J \eta^{t} J^{-1} \text { where } J=\left(\begin{array}{ccccc}
0 & 0 & \ldots & 0 & 1 \\
0 & 0 & \ldots & 1 & 0 \\
\ldots & \ldots & \ldots & \ldots & \ldots \\
0 & 1 & \ldots & 0 & 0 \\
1 & 0 & \ldots & 0 & 0
\end{array}\right) \text {. }
$$

They act on the standard simple root system $\left\{\alpha_{1}=\epsilon_{1}-\epsilon_{2}, \ldots, \alpha_{d-1}=\epsilon_{d-1}-\epsilon_{d}\right\}$ by $\alpha_{i} \mapsto \alpha_{d-i}$. Define

$$
\begin{aligned}
& \phi_{n+1, n}: G_{n} \rightarrow G_{n+1} \text { by } \phi_{n+1, n}(g)=\operatorname{diag}\{g, \ldots, g ; \psi(g), \ldots, \psi(g) ; 1, \ldots, 1\}, \\
& d \phi_{n+1, n}: \mathfrak{g}_{n} \rightarrow \mathfrak{g}_{n+1} \text { by } d \phi_{n+1, n}(\eta)=\operatorname{diag}\{\eta, \ldots, \eta ; d \psi(\eta), \ldots, d \psi(\eta) ; 0, \ldots, 0\}
\end{aligned}
$$

with $r_{n+1}$ blocks $g$, with $s_{n+1}$ blocks $\psi(g)$, and with $t_{n+1}$ entries 1 . The point of $\psi(g)$ instead of $\left(g^{t}\right)^{-1}$ here is that $d \phi_{n+1, n}$ sends diagonal matrices to diagonal matrices and sends upper triangular matrices to upper triangular matrices, so (0.2) holds. Thus our basic setup is valid for

$$
\begin{aligned}
G_{u} & =S U_{\mathbf{r}, \mathbf{s}, \mathbf{t}}(\infty)=\lim _{\longrightarrow}\left\{S U\left(d_{n}\right), \phi_{m, n}\right\} \text { and } \\
G & =S L_{\mathbf{r}, \mathbf{s}, \mathbf{t}}(\infty ; \mathbb{C})=\underline{\lim }\left\{S L\left(d_{n} ; \mathbb{C}\right), \phi_{m, n}\right\}
\end{aligned}
$$

where, of course, $\phi_{m, n}=\phi_{m, m-1} \cdot \phi_{m-1, m-2} \cdots \phi_{n+1, n}$ for $m>n$.

One can, of course, do the same thing with $G_{u, n}=U\left(d_{n}\right)$ and $G_{n}=G L\left(d_{n} ; \mathbb{C}\right)$, obtaining $U_{\mathbf{r}, \mathbf{s}, \mathbf{t}}(\infty)=\lim _{\longrightarrow}\left\{U\left(d_{n}\right), \phi_{m, n}\right\}, G L_{\mathbf{r}, \mathbf{s}, \mathbf{t}}(\infty ; \mathbb{C})=\lim _{\longrightarrow}\left\{G L\left(d_{n} ; \mathbb{C}\right), \phi_{m, n}\right\}$. 
At that point one can replace some or all of the 1's for $\phi_{n+1, n}$ in (5.1b) by various powers of $\operatorname{det} g$, and the corresponding 0 's for $d \phi_{n+1, n}$ by the corresponding multiples of trace $\eta$. See the Escher embeddings of Section 6 .

Type BD. We look at diagonal embeddings of orthogonal groups. Again we fix sequences $\mathbf{r}=\left\{r_{n}\right\}_{n \geqq 0}, \mathbf{s}=\left\{s_{n}\right\}_{n \geqq 0}$ and $\mathbf{t}=\left\{t_{n}\right\}_{n \geqq 0}$ of non-negative integers with $r_{0}>0=s_{0}=t_{0}$. Let $d_{0}=r_{0}, G_{u, 0}=S O\left(d_{0}\right)$, and $G_{0}=S O\left(d_{0} ; \mathbb{C}\right)$. Recursively define $G_{u, n+1}=S O\left(d_{n+1}\right)$ and $G_{n+1}=S O\left(d_{n+1} ; \mathbb{C}\right)$ where (i) if $d_{n}$ is even then $d_{n+1}=d_{n}\left(r_{n+1}+s_{n+1}\right)+t_{n+1}$, (ii) if $d_{n}$ is odd then $d_{n+1}=d_{n} r_{n+1}+t_{n+1}$. Thus we may, and do, assume $s_{n+1}=0$ whenever $d_{n}$ is odd. For $d$ even, say $d=2 u$, we consider the outer automorphisms $\psi$ of $S O(d ; \mathbb{C})$ and $d \psi$ of $\mathfrak{s o}(d ; \mathbb{C})$ given by

$$
\psi(g)=D g D^{-1} \text { and } d \psi(\eta)=D \eta D^{-1} \text { where } D=\operatorname{diag}\{1, \cdots, 1,-1\} .
$$

Let $\xi_{i}=\operatorname{diag}\left\{0_{2}, \cdots, 0_{2}, J_{2}, 0_{2}, \cdots, 0_{2}\right\}$ where $0_{2}$ is the $2 \times 2$ zero matrix and $J_{2}=\left(\begin{array}{cc}0 & 1 \\ -1 & 0\end{array}\right)$ is the $i$ th diagonal block. Then $\left\{\xi_{1}, \ldots, \xi_{u}\right\}$ is the usual basis of the usual Cartan subalgebra of $\mathfrak{s o}(d ; \mathbb{C})$, dual to the linear functionals $\left\{\epsilon_{i}\right\}$ such that the $\alpha_{1}=\epsilon_{1}-\epsilon_{2}, \ldots, \alpha_{d-1}=\epsilon_{d-1}-\epsilon_{d}$ and $\alpha_{d}=\epsilon_{d-1}+\epsilon_{d}$ form the standard simple root system. Note that $A \mapsto \hat{A}$ induces the signed permutation

$$
\xi_{1} \mapsto \xi_{1}, \ldots, \xi_{d-1} \mapsto \xi_{d-1}, \quad \xi_{d} \mapsto-\xi_{d}
$$

and thus induces the permutation

$$
\alpha_{1} \mapsto \alpha_{1}, \quad \ldots, \alpha_{d-2} \mapsto \alpha_{d-2}, \quad \alpha_{d-1} \mapsto \alpha_{d}, \quad \alpha_{d} \mapsto \alpha_{d-1}
$$

on the simple root system. Again define

$$
\begin{aligned}
& \phi_{n+1, n}: G_{n} \rightarrow G_{n+1} \text { by } \phi_{n+1, n}(g)=\operatorname{diag}\{g, \ldots, g ; \psi(g), \ldots, \psi(g) ; 1, \ldots, 1\}, \\
& d \phi_{n+1, n}: \mathfrak{g}_{n} \rightarrow \mathfrak{g}_{n+1} \text { by } d \phi_{n+1, n}(\eta)=\operatorname{diag}\{\eta, \ldots, \eta ; d \psi(\eta), \ldots, d \psi(\eta) ; 0, \ldots, 0\}
\end{aligned}
$$

with $r_{n+1}$ blocks $g$, with $s_{n+1}$ blocks $\psi(g)$, and with $t_{n+1}$ entries 1 . Recall $s_{n+1}=0$ if $d_{n}$ is odd, so the $\psi(g)$ only occur when they are defined. As just discussed, $\phi_{n+1, n}$ satisfies (0.2). Thus our basic setup is valid for

$$
\begin{aligned}
G_{u} & =S O_{\mathbf{r}, \mathbf{s}, \mathbf{t}}(\infty)=\underline{\lim }\left\{S O\left(d_{n}\right), \phi_{m, n}\right\} \text { and } \\
G & =S O_{\mathbf{r}, \mathbf{s}, \mathbf{t}}(\infty ; \mathbb{C})=\underline{\varliminf}\left\{S O\left(d_{n} ; \mathbb{C}\right), \phi_{m, n}\right\}
\end{aligned}
$$

where, as before, $\phi_{m, n}=\phi_{m, m-1} \cdot \phi_{m-1, m-2} \cdots \phi_{n+1, n}$ for $m>n$.

One can, of course, do the same thing with $G_{u, n}=O\left(d_{n}\right)$ and $G_{n}=O\left(d_{n} ; \mathbb{C}\right)$, obtaining $O_{\mathbf{r}, \mathbf{s}, \mathbf{t}}(\infty)=\varliminf_{\longrightarrow}\left\{U\left(d_{n}\right), \phi_{m, n}\right\}$ and $O_{\mathbf{r}, \mathbf{s}, \mathbf{t}}(\infty ; \mathbb{C})=\varliminf_{\longrightarrow}\left\{O\left(d_{n} ; \mathbb{C}\right), \phi_{m, n}\right\}$. At that point one can replace some or all of the 1's for $\phi_{n+1, n}$ in (5.2b) by $\operatorname{det} g$, and the corresponding 0 's for $d \phi_{n+1, n}$ by trace $\eta$.

Type C. We look at diagonal embeddings of symplectic groups. Fix sequences $\mathbf{r}=\left\{r_{n}\right\}_{n \geqq 0}$ and $\mathbf{t}=\left\{t_{n}\right\}_{n \geqq 0}$ of non-negative integers with $r_{0}>0=t_{0}$. We do not need a sequence $\mathbf{s}$, though of course we may think of it as all zeros, because every automorphism of $S p(d)$ is inner. We use the convention that $S p(d)$ is the unitary symplectic group acting on quaternionic $n$-space or complex $2 n$-space, and $S p(d, \mathbb{C})$ is its complexification.

Let $d_{0}=r_{0}, G_{u, 0}=S p\left(d_{0}\right)$, and $G_{0}=S p\left(d_{0} ; \mathbb{C}\right)$. Recursively define $G_{u, n+1}=$ $S p\left(d_{n+1}\right)$ and $G_{n+1}=S p\left(d_{n+1} ; \mathbb{C}\right)$ where $d_{n+1}=d_{n} r_{n+1}+t_{n+1}$. Define

$$
\begin{aligned}
& \phi_{n+1, n}: G_{n} \rightarrow G_{n+1} \text { by } \phi_{n+1, n}(g)=\operatorname{diag}\{g, \ldots, g ; 1, \ldots, 1\}, \\
& d \phi_{n+1, n}: \mathfrak{g}_{n} \rightarrow \mathfrak{g}_{n+1} \text { by } d \phi_{n+1, n}(\eta)=\operatorname{diag}\{\eta, \ldots, \eta ; 0, \ldots, 0\}
\end{aligned}
$$


with $r_{n+1}$ blocks $g$ and $t_{n+1}$ entries 1 . Now $\phi_{n+1, n}$ satisfies (0.2), so our basic setup is valid for

$$
\begin{aligned}
G_{u} & =S p_{\mathbf{r}, \mathbf{t}}(\infty)=\underline{\lim }\left\{S p\left(d_{n}\right), \phi_{m, n}\right\} \text { and } \\
G & =S p_{\mathbf{r}, \mathbf{t}}(\infty ; \mathbb{C})=\underline{\lim _{\longrightarrow}}\left\{S p\left(d_{n} ; \mathbb{C}\right), \phi_{m, n}\right\}
\end{aligned}
$$

where, as usual, $\phi_{m, n}=\phi_{m, m-1} \cdot \phi_{m-1, m-2} \cdots \phi_{n+1, n}$ for $m>n$.

The standard embedding direct limits of $\S 8$ are the cases of (5.1), (5.2) and (5.3) where $r_{i}=1, s_{i}=0$ and $t_{i}=1$. There are many other interesting patterns such as the doubling embeddings where $r_{i}=2$ and $s_{i}=0=t_{i}$, the tripling embeddings where $r_{i}=3$ and $s_{i}=0=t_{i}$, etc.

For convenience of exposition we always assume that $r_{i}+s_{i}>0$. This has no effect on the limit, as one can simply increase the next $t_{j}$.

At this point we have to fix some notation for Cartan subalgebras and roots. We fix systems of Cartan subalgebras and linear functionals. Let $J=\left(\begin{array}{cc}0 & 1 \\ -1 & 0\end{array}\right)$. The usual system is

$$
\begin{aligned}
\text { Type } A_{\ell}: \mathfrak{h}_{n}= & \left\{\operatorname{diag}\left\{a_{1}, \ldots, a_{\ell+1}\right\} \mid a_{k} \in \mathbb{C}, \sum a_{k}=0\right\}, \\
& \epsilon_{k}\left(\operatorname{diag}\left\{a_{1}, \ldots, a_{\ell+1}\right\}\right)=a_{k}, \\
\text { Type } B_{\ell}: \mathfrak{h}_{n}= & \left\{\operatorname{diag}\left\{a_{1} J, \ldots, a_{\ell} J, 0\right\} \mid a_{k} \in \mathbb{C}\right\}, \\
& \epsilon_{k}\left(\operatorname{diag}\left\{a_{1} J, \ldots, a_{\ell} J, 0\right\}\right)=\sqrt{-1} a_{k}, \\
\text { Type } C_{\ell}: \mathfrak{h}_{n}= & \left\{\operatorname{diag}\left\{a_{1},-a_{1}, \ldots, a_{\ell},-a_{-\ell}\right\} \mid a_{k} \in \mathbb{C}\right\}, \\
& \epsilon_{k}\left(\operatorname{diag}\left\{a_{1},-a_{1}, \ldots, a_{\ell},-a_{-\ell}\right\}\right)=a_{k}, \\
\text { Type } D_{\ell}: \mathfrak{h}_{n}= & \left\{\operatorname{diag}\left\{a_{1} J, \ldots, a_{\ell} J\right\} \mid a_{k} \in \mathbb{C}\right\}, \\
& \epsilon_{k}\left(\operatorname{diag}\left\{a_{1} J, \ldots, a_{\ell} J\right\}\right)=\sqrt{-1} a_{k} .
\end{aligned}
$$

For pure type $B$ it will sometimes be useful to think in terms of an alternative system

$$
\text { Type } B_{\ell}: \quad \begin{aligned}
\mathfrak{h}_{n}^{\prime}= & \left\{\operatorname{diag}\left\{0, a_{1} J, \ldots, a_{\ell} J\right\} \mid a_{k} \in \mathbb{C}\right\}, \\
& \epsilon_{k}^{\prime}\left(\operatorname{diag}\left\{0, a_{1} J, \ldots, a_{\ell} J\right\}\right)=\sqrt{-1} a_{k} .
\end{aligned}
$$

The root system $\Delta_{n}=\Delta\left(\mathfrak{g}_{n}, \mathfrak{h}_{n}\right)$ is

$$
\begin{array}{lll}
\text { Type } A_{\ell}: & \Delta_{n}=\left\{ \pm\left(\epsilon_{i}-\epsilon_{j}\right), 1 \leqq i<j \leqq \ell+1\right\} & \text { with } d_{n}=\ell+1 \\
\text { Type } B_{\ell}: & \Delta_{n}=\left\{ \pm\left(\epsilon_{i} \pm \epsilon_{j}\right) \text { and } \pm \epsilon_{i}, 1 \leqq i<j \leqq \ell\right\} & \text { with } d_{n}=2 \ell+1, \\
\text { Type } C_{\ell}: & \Delta_{n}=\left\{ \pm\left(\epsilon_{i} \pm \epsilon_{j}\right) \text { and } \pm 2 \epsilon_{i}, 1 \leqq i<j \leqq \ell\right\} & \text { with } d_{n}=2 \ell \\
\text { Type } D_{\ell}: & \Delta_{n}=\left\{ \pm\left(\epsilon_{i} \pm \epsilon_{j}\right), 1 \leqq i<j \leqq \ell\right\} & \text { with } d_{n}=2 \ell
\end{array}
$$

We think of the usual positive root system $\Delta_{n}^{+}=\Delta^{+}\left(\mathfrak{g}_{n}, \mathfrak{h}_{n}\right)$ as defined by its simple root subsystem $\Psi_{n}=\Psi\left(\mathfrak{g}_{n}, \mathfrak{h}_{n}\right)$, as follows. Here we also record $\rho_{n}$, half the sum of the positive roots.

$$
\begin{aligned}
\text { Type } A_{\ell}: \Psi_{n}= & \left\{\epsilon_{k}-\epsilon_{k+1} \mid 1 \leqq k \leqq \ell\right\}, \\
& \rho_{n}=\sum_{k=1}^{\ell+1}\left(\frac{\ell}{2}+1-k\right) \epsilon_{k}, \\
\text { Type } B_{\ell}: \Psi_{n}= & \left\{\epsilon_{k}-\epsilon_{k+1} \mid 1 \leqq k<\ell\right\} \cup\left\{\epsilon_{\ell}\right\}, \\
& \rho_{n}=\sum_{k=1}^{\ell}\left(\ell+\frac{1}{2}-k\right) \epsilon_{k}, \\
\text { Type } C_{\ell}: \Psi_{n}= & \left\{\epsilon_{k}-\epsilon_{k+1} \mid 1 \leqq k<\ell\right\} \cup\left\{2 \epsilon_{\ell}\right\}, \\
& \rho_{n}=\sum_{k=1}^{\ell}(\ell+1-k) \epsilon_{k}, \\
\text { Type } D_{\ell}: \Psi_{n}= & \left\{\epsilon_{k}-\epsilon_{k+1} \mid 1 \leqq k<\ell\right\} \cup\left\{\epsilon_{\ell-1}+\epsilon_{\ell}\right\}, \\
& \rho_{n}=\sum_{k=1}^{\ell}(\ell-k) \epsilon_{k} .
\end{aligned}
$$


These positive root systems satisfy (0.2) but are not nicely aligned for pull-back of simple roots. For that, it is better to use the positive root system ' $\Delta_{n}^{+}=$ ${ }^{\prime} \Delta^{+}\left(\mathfrak{g}_{n}, \mathfrak{h}_{n}\right)$, defined by its simple root subsystem ' $\Psi_{n}={ }^{\prime} \Psi\left(\mathfrak{g}_{n}, \mathfrak{h}_{n}\right)$ as follows.

$$
\begin{aligned}
\text { Type } A_{\ell}:{ }^{\prime} \Psi_{n}= & \left\{\epsilon_{k+1}-\epsilon_{k} \mid 1 \leqq k \leqq \ell\right\}, \\
& \rho_{n}=\sum_{k=1}^{\ell+1}\left(k-\frac{\ell}{2}-1\right) \epsilon_{k}, \\
\text { Type } B_{\ell}:{ }^{\prime} \Psi_{n}= & \left\{\epsilon_{k+1}-\epsilon_{k} \mid 1 \leqq k<\ell\right\} \cup\left\{\epsilon_{1}\right\}, \\
& \rho_{n}=\sum_{k=1}^{\ell}\left(k-\frac{1}{2}\right) \epsilon_{k}, \\
\text { Type } C_{\ell}:{ }^{\prime} \Psi_{n}= & \left\{\epsilon_{k+1}-\epsilon_{k} \mid 1 \leqq k<\ell\right\} \cup\left\{2 \epsilon_{1}\right\}, \\
& \rho_{n}=\sum_{k=1}^{\ell} k \epsilon_{k}, \\
\text { Type } D_{\ell}:{ }^{\prime} \Psi_{n}= & \left\{\epsilon_{k+1}-\epsilon_{k} \mid 1 \leqq k<\ell\right\} \cup\left\{\epsilon_{2}+\epsilon_{1}\right\}, \\
& ' \rho_{n}=\sum_{k=1}^{\ell}(k-1) \epsilon_{k} .
\end{aligned}
$$

It is immediate from the constructions (5.1), (5.2) and (5.3), that, either using (5.7) or using (5.8) for positive root system, we have

5.9. Proposition. The diagonal embedding direct limit systems (5.1), (5.2) and (5.3) satisfy the basic alignment conditions (0.1), (0.2) and (0.3). In particular the Borel-Weil Theorem 3.13 applies to those limit groups.

The basic alignment condition (7.1) holds if and only if the $r_{i}+s_{i}=1$, so we must examine the precise conditions of the Bott-Borel-Weil Theorem 4.6.

Consider the inverse limit

$$
\Delta=\Delta(\mathfrak{g}, \mathfrak{h})=\varliminf_{\lim }\left(\Delta\left(\mathfrak{g}_{\alpha}, \mathfrak{h}_{\alpha}\right), d \phi_{\beta, \alpha}^{*}\right) .
$$

Here it is understood that threads only exist above some point. Thus, if $\delta \in \Delta$, say $\delta=\left[\delta_{\alpha}\right]$, then there is an index $\alpha_{0}=\alpha_{0}(\delta)$ such that $d \phi_{\beta, \alpha}^{*}\left(\delta_{\beta}\right)$ is guaranteed to be defined only when $\beta \geqq \alpha \geqq \alpha_{0}(\delta)$. We will not have uniqueness such as that of Lemma 7.3 unless $r_{i}+s_{i}=1$, so the inverse limit $\Delta$ of (5.10) will generally be larger than the direct limit $\Delta_{F}$ of (7.4). In fact, if we have $r_{i}+s_{i}>1$ infinitely often, then $\Delta^{+}$will be uncountable. In any case it is important not to confuse $\Delta$ with $\Delta_{F}$.

5.11. Definition. A diagonal embedding direct limit system (5.1), (5.2) and (5.3) is of classical type if, for some index $i_{0}, r_{i}+s_{i}=1$ whenever $i \geqq i_{0}$. If it is not of classical type, in other words if $r_{i}+s_{i}>1$ infinitely often, then it is of non-classical type.

Let $\mathfrak{g}=\lim _{\mathfrak{g}} \mathfrak{g}_{n}$ be one of the three types (5.1), (5.2) or (5.3) of diagonally embedded direct limit systems. Suppose that it is of classical type. The root system $\Delta_{n}=\Delta\left(\mathfrak{g}_{n}, \mathfrak{h}_{n}\right)$ is given by (5.6). In each case let $\epsilon_{k} \in \mathfrak{h}^{*}$ denote the linear functional that sends $d \phi_{n}(\eta) \in \mathfrak{h}$ to $\epsilon_{k}(\eta)$, computed in $\mathfrak{h}_{n}$, for $n$ sufficiently large. Here, if $m \geqq n$ with $n$ sufficiently large, every root $\nu_{n} \in \Delta_{n}$ is of the form $d \phi_{m, n}^{*}\left(\nu_{m}\right)$ for just one $\nu_{m} \in \Delta_{m}$. From this the inverse limit (5.10) is in effect a union when $\mathfrak{g}=\lim _{\longrightarrow} \mathfrak{g}_{n}$ is of classical type. Thus

5.12. Proposition. Let $\mathfrak{g}=\lim _{\longrightarrow} \mathfrak{g}_{n}$ be a diagonal embedding direct limit system (5.1), (5.2) or (5.3) of classical type. Then the root system

$$
\Delta=\Delta(\mathfrak{g}, \mathfrak{h})=\bigcup \Delta\left(\mathfrak{g}_{n}, \mathfrak{h}_{n}\right)
$$


in the sense that it is given by

$$
\begin{array}{ll}
\text { Type A: } & \Delta(\mathfrak{g}, \mathfrak{h})=\left\{ \pm\left(\epsilon_{i}-\epsilon_{j}\right), 1 \leqq i<j<\infty\right\} \\
\text { Type } B: & \Delta(\mathfrak{g}, \mathfrak{h})=\left\{ \pm\left(\epsilon_{i} \pm \epsilon_{j}\right) \text { and } \pm \epsilon_{i}, 1 \leqq i<j<\infty\right\} \\
\text { Type } C: & \Delta(\mathfrak{g}, \mathfrak{h})=\left\{ \pm\left(\epsilon_{i} \pm \epsilon_{j}\right) \text { and the } \pm 2 \epsilon_{i}, 1 \leqq i<j<\infty\right\}, \\
\text { Type } D: & \Delta(\mathfrak{g}, \mathfrak{h})=\left\{ \pm\left(\epsilon_{i} \pm \epsilon_{j}\right), 1 \leqq i<j<\infty\right\} .
\end{array}
$$

There is a root space decomposition $\mathfrak{g}=\mathfrak{h}+\sum_{\alpha \in \Delta} \mathfrak{g}_{\alpha}$, and the Weyl group $W(\mathfrak{g}, \mathfrak{h})$ of (4.4) is given by

Type A: all permutations of the $\epsilon_{i}$ that preserve each $\left\{\epsilon_{1} \ldots, \epsilon_{d_{n}}\right\}$ for $n \gg 0$,

Type B: all signed permutations of the $\epsilon_{i}$ that preserve each $\left\{ \pm \epsilon_{1} \ldots, \pm \epsilon_{d_{n}}\right\}$ for $n \gg 0$,

Type C: all signed permutations of the $\epsilon_{i}$ that preserve each $\left\{ \pm \epsilon_{1} \ldots, \pm \epsilon_{d_{n}}\right\}$ for $n \gg 0$,

Type D: all signed permutations of the $\epsilon_{i}$

that preserve each $\left\{ \pm \epsilon_{1} \ldots, \pm \epsilon_{d_{n}}\right\}$ for $n \gg 0$, where the number of sign changes on $\left\{\epsilon_{1} \ldots, \epsilon_{d_{n}}\right\}$ is even for $n \gg 0$.

The finite Weyl group situation is more delicate. It clearly distinguishes between the root orders of (5.7) and (5.8). Denote

$$
\begin{aligned}
\mathfrak{b} & =\mathfrak{h}+\sum_{\nu \in \Delta^{+}} \mathfrak{g}_{-\nu} \quad \text { Borel for (5.7) } \quad \text { and } \\
\mathfrak{b} & =\mathfrak{h}+\sum_{\nu \in \in^{\prime} \Delta^{+}} \mathfrak{g}_{-\nu} \quad \text { Borel for (5.8). }
\end{aligned}
$$

5.14. Proposition. Let $\mathfrak{g}=\lim _{\mathfrak{g}} \mathfrak{g}_{n}$ be a diagonal embedding direct limit system (5.1), (5.2) or (5.3) of classical type. Then $W_{F}(\mathfrak{g}, \mathfrak{b}, \mathfrak{h})$ is given by all finitely supported permutations $w$ (without any sign changes) of the $\epsilon_{k}$, in other words all permutations $w$ such that, for some $n_{0}=n_{0}(w)>0$, we have $w\left(\epsilon_{k}\right)=\epsilon_{k}$ whenever $k \geqq n_{0}$. And $W_{F}(\mathfrak{g}, ' \mathfrak{b}, \mathfrak{h})$ is given by

Type A: all finitely supported permutations of the $\epsilon_{k}$,

Type B: all finitely supported signed permutations of the $\epsilon_{k}$,

Type C: all finitely supported signed permutations of the $\epsilon_{k}$,

Type D: all finitely supported signed permutations of the $\epsilon_{k}$

where the number of sign changes is even.

In Section 8 we will describe a more general characterization of $W_{F}$ and will see how the second part of Proposition 5.14 can be formulated as $W_{F}\left(\mathfrak{g},{ }^{\prime} \mathfrak{b}, \mathfrak{h}\right)=$ $\stackrel{\lim }{\longrightarrow}\left(\mathfrak{g}_{n}, \mathfrak{b}_{n}, \mathfrak{h}_{n}\right)$.

Proof. The statement concerning $W_{F}(\mathfrak{g}, \mathfrak{b}, \mathfrak{h})$ is straightforward. In effect, each simple $\psi_{n} \in{ }^{\prime} \Psi_{n}$ has unique expression $\psi_{n}=d \phi_{m, n}^{*}\left(\nu_{m}\right)$ with $\nu_{m} \in \Delta_{m}$ for $m \geqq n$, and here $\nu_{m}$ is some $\psi_{m} \in{ }^{\prime} \Psi_{m}$. So if $w \in W$ is finitely supported and $w_{n}$ has a given reduced expression $s_{\psi_{n, 1}} \ldots s_{\psi_{n, \ell}}$ as product of simple root reflections, i.e. $\psi_{n, k} \in \Psi_{n}$, and if $m \geqq n$, then $w_{m}$ has reduced expression $s_{\psi_{m, 1}} \ldots s_{\psi_{m, \ell}}$ of the same length where $\psi_{m, k} \in \Psi_{m}$ and $d \phi_{m, n}^{*}\left(\psi_{m, k}\right)=\psi_{n, k}$. Thus every $w \in$ $W_{F}(\mathfrak{g}, \mathfrak{b}, \mathfrak{h})$ is finitely supported. Conversely if $w \in W(\mathfrak{g}, \mathfrak{h})$ is finitely supported 
then a computation shows that $w \in W_{F}\left(\mathfrak{g},{ }^{\prime} \mathfrak{b}, \mathfrak{h}\right)$. The assertion on $W_{F}\left(\mathfrak{g},{ }^{\prime} \mathfrak{b}, \mathfrak{h}\right)$ now follows from the (signed) permutation description of the $W\left(\mathfrak{g}_{n}, \mathfrak{h}_{n}\right)$.

Now consider $W_{F}=W_{F}(\mathfrak{g}, \mathfrak{b}, \mathfrak{h})$. If $w$ is a permutation without any sign changes and $w\left(\epsilon_{i}\right)=\epsilon_{i}$ for $i \geqq n_{0}$ then $w \in W_{F}$ directly from the definition. Conversely suppose $w \in W_{F}$. Decompose $w=w_{s} w_{p}$ where $w_{p}$ permutes the $\left\{\epsilon_{i}\right\}$ and $w_{s}$ gives the sign changes. We first show $w_{s}=1$. Of course this is automatic for diagonal embedding direct limit groups of Type A, so we suppose that $G$ is of Type B, C or D. As $\ell(w)<\infty$ we have $w_{s}\left(\epsilon_{i}\right)=-\epsilon_{i}$ for only finitely many indices $i$. Choose one such index $i$ and let $u$ be the index such that $w\left(\epsilon_{u}\right)=-\epsilon_{i}$. There are infinitely many indices $v>u$ such that $w\left(\epsilon_{v}\right)$ is of the form $\epsilon_{j}$ with $j>i$. For each such index $v$, the root $\epsilon_{u}-\epsilon_{v}$ is positive while $w\left(\epsilon_{u}-\epsilon_{v}\right)=-\epsilon_{i}-\epsilon_{j}$ is negative. That contradicts $\ell(w)<\infty$. Thus we cannot make a choice of index $i$, in other words $w_{s}=1$. We have proved the statement characterizing $W_{F}(\mathfrak{g}, \mathfrak{b}, \mathfrak{h})$.

5.15. Proposition. Let $\mathfrak{g}=\lim _{\mathfrak{g}} \mathfrak{g}_{n}$ be a diagonal embedding direct limit system (5.1), (5.2) or (5.3) of classical type. Let $\lambda=\sum m_{i} \epsilon_{i} \in \mathfrak{h}^{*}, m_{i}$ real. Then the following conditions are equivalent.

(i) $\lambda$ is cohomologically finite,

(ii) $\lambda$ is classically cohomologically finite, and

(iii) Root order (5.7): the $m_{i}$ are integers, $m_{i} \neq m_{i+1}-1$ for all $i, m_{i} \geqq m_{i+1}$ with only finitely many exceptions, and for Types $B, C$ and $D$ all $m_{i} \geqq 0$.

Root order (5.8): the $m_{i}$ are integers, $m_{i} \neq m_{i+1}+1$ for all $i, m_{i} \leqq m_{i+1}$ with only finitely many exceptions, and for Types $B, C$ and $D$ all but finitely many $m_{i} \geqq 0$.

Proof. First suppose that we are in the case of root order (5.7). We show that (i) implies (ii). Let $\lambda$ be cohomologicaly finite of degree $q_{\lambda}$. For $n$ sufficiently large we have a unique Weyl group element $w_{n} \in W\left(\mathfrak{g}_{n}, \mathfrak{h}_{n}\right)$ of Weyl group length $q_{\lambda}$ such that $w_{n}\left(\lambda_{n}+\rho_{n}\right)-\rho_{n}$ is dominant. This dominance means $\left\langle w_{n}\left(\lambda_{n}+\rho_{n}\right)-\rho_{n}, \epsilon_{i}\right\rangle \geqq$ $\left\langle w_{n}\left(\lambda_{n}+\rho_{n}\right)-\rho_{n}, \epsilon_{i+1}\right\rangle$ for $1 \leqq i \leqq \ell$ for Type $A_{\ell},\left\langle w_{n}\left(\lambda_{n}+\rho_{n}\right)-\rho_{n}, \epsilon_{i}\right\rangle \geqq$ $\left\langle w_{n}\left(\lambda_{n}+\rho_{n}\right)-\rho_{n}, \epsilon_{i+1}\right\rangle$ for $1 \leqq i<\ell$ and $\left\langle w_{n}\left(\lambda_{n}+\rho_{n}\right)-\rho_{n}, \epsilon_{\ell}\right\rangle \geqq 0$ for Types $B_{\ell}, C_{\ell}$ and $D_{\ell}$. For $m \geqq n, w_{m}$ has the same Weyl group length $q_{\lambda}$ as $w_{n}$ and also satisfies these dominance conditions.

The Cartan-Killing form of $\mathfrak{g}_{m}$ restricts (by means of $d \phi_{m, n}$ ) to an invariant bilinear form on $\mathfrak{g}_{n}$, thus to a (necessarily positive) multiple of the Cartan-Killing form of $\mathfrak{g}_{n}$. We identify the Cartan subalgebras with their duals, $b_{k}: \mathfrak{h}_{k}^{*} \cong \mathfrak{h}_{k}$, and that identification $b_{k}$ is essentially invariant under our inclusions because $d \phi_{m, n}^{*}$. $b_{n} \cdot d \phi_{m, n}=c_{m, n} b_{m}$ for some $c_{m, n}>0$. Later we will formalize this situation as alignment $(7.1 \mathrm{~b})$ of the Cartan-Killing forms. Anyway, now we see that $w_{m}$ preserves $\mathfrak{h}_{n}^{*}=d \phi_{m, n}^{*}\left(\mathfrak{h}_{m}^{*}\right)$, and the restriction of $w_{m}$ to $\mathfrak{h}_{n}^{*}$ is given by $w_{n}$. More precisely, $d \phi_{m, n}^{*} \cdot w_{m}=w_{n} \cdot d \phi_{m, n}^{*}$

In view of the last paragraph of the proof of Proposition 5.14 we conclude that $w_{m}$ acts only by permutations - no sign changes - on the $\epsilon_{i}$. Thus we have $w \in W_{F}$ such that $w_{n}=\left.w\right|_{\mathfrak{h}_{n}}$ for $n$ sufficiently large. The length $\ell(w)$ is the cardinality of

$$
\mathcal{N}_{n}(w)=\left\{\nu_{n} \in \Delta_{n}^{+} \mid w_{n}\left(\nu_{n}\right) \in-\Delta_{n}^{+}\right\}
$$


for $n \geqq n_{0}=n_{0}(w)$. If $m \geqq n \geqq n_{0}$ then $d \phi_{m, n}^{*}\left(\mathcal{N}_{m}(w)\right)=\mathcal{N}_{n}(w)$. Thus $\mathcal{N}_{n}(w)$ has exactly $\ell(w)$ elements and, in fact, $w_{n}\left(\rho_{n}\right)-\rho_{n}=-\sum_{\nu_{n} \in \mathcal{N}_{n}(w)} \nu_{n}$. This proves:

$$
\text { if } m \geqq n \gg 0 \text {, then } d \phi_{m, n}^{*}\left(w_{m}\left(\rho_{m}\right)-\rho_{m}\right)=w_{n}\left(\rho_{n}\right)-\rho_{n} .
$$

In particular, as $\lambda \in \mathfrak{h}^{*}$ is integral and satisfies

$$
\left\langle w_{n}\left(\lambda_{n}+\rho_{n}\right), \nu_{n}\right\rangle>0 \text { for } n \gg 0 \text { and } \nu_{n} \in \Delta_{n}^{+}
$$

where $\lambda_{n}=d \phi_{n}^{*}(\lambda)$, there is a dominant integral element $\widetilde{\lambda} \in \mathfrak{h}^{*}$ such that

$$
d \phi_{n}^{*}(\widetilde{\lambda})=w_{n}\left(\lambda_{n}+\rho_{n}\right)-\rho_{n} \text { for } n \gg 0 .
$$

This completes the proof that (i) implies (ii) for root order (5.7).

We show that (ii) implies (iii). The $m_{i}$ are integers because $\lambda$ is integral. In view of (5.7), the condition $m_{i} \neq m_{i+1}-1$ just says that $\left\langle\lambda_{n}+\rho_{n}, \epsilon_{i}-\epsilon_{i+1}\right\rangle \neq 0$, which follows from the nonsingularity of $\lambda_{n}+\rho_{n}$ that is built into the definition of cohomological finiteness. A real functional $\lambda^{\prime}=\sum m_{i}^{\prime} \epsilon_{i}$ is dominant if and only the following are satisfied. For Type A: $m_{i}^{\prime} \geqq m_{i+i}^{\prime}$ for all $i$, so every $\left\langle\lambda^{\prime}, \epsilon_{i}-\epsilon_{i+1}\right\rangle \geqq 0$. For Types B, C and D: $m_{i}^{\prime} \geqq m_{i+i}^{\prime}$ for all $i$, so every $\left\langle\lambda^{\prime}, \epsilon_{i}-\epsilon_{i+1}\right\rangle \geqq 0$, and also $m_{i}^{\prime} \geqq 0$ for all $i$, so every $\left\langle\lambda^{\prime}, \epsilon_{i}\right\rangle \geqq 0$. This completes the proof that (ii) implies (iii) for root order (5.7).

Now suppose that we are in the case of root order (5.8). The argument that (i) implies (ii) is exactly the same as for root order (5.7) except that $w_{m}$ may incorporate sign changes. In view of Proposition 5.14 we still have $w \in W_{F}$ such that $w_{n}=\left.w\right|_{\mathfrak{h}_{n}}$ for $n$ sufficiently large. The argument now continues as for root order (5.7). Thus (i) implies (ii) for root order (5.8).

We show that (ii) implies (iii). The $m_{i}$ are integers because $\lambda$ is integral. In view of (5.8), the condition $m_{i} \neq m_{i+1}+1$ just says that $\left\langle\lambda_{n}+\rho_{n}, \epsilon_{i+i}-\epsilon_{i}\right\rangle \neq 0$, which follows from the nonsingularity of $\lambda_{n}+\rho_{n}$ that is built into the definition of cohomological finiteness. A real functional $\lambda^{\prime}=\sum m_{i}^{\prime} \epsilon_{i}$ is dominant if and only the following are satisfied. For Type A: $m_{i}^{\prime} \leqq m_{i+i}^{\prime}$ for all $i$, so every $\left\langle\lambda^{\prime}, \epsilon_{i+1}-\epsilon_{i}\right\rangle \geqq 0$. For Types B, C and D: $m_{i}^{\prime} \leqq m_{i+i}^{\prime}$ for all $i$, so every $\left\langle\lambda^{\prime}, \epsilon_{i+1}-\epsilon_{i}\right\rangle \geqq 0$, and also $m_{i}^{\prime} \geqq 0$ for all $i$, so every $\left\langle\lambda^{\prime}, \epsilon_{i}\right\rangle \geqq 0$. This completes the proof that (ii) implies (iii) for root order (5.7).

It is immediate from (5.7), (5.8) and Proposition 5.14 that (iii) implies (ii) and thus implies (i). That completes the proof of Proposition 5.15.

The situation in the non-classical case is given by

5.17. Proposition. Let $\mathfrak{g}=\lim _{\mathfrak{g}} \mathfrak{g}_{n}$ be a diagonal embedding direct limit system (5.1), (5.2) or (5.3) of non-classical type. Then the root system $\Delta=\Delta(\mathfrak{g}, \mathfrak{h})$ is uncountable and $\mathfrak{g}$ has no root space decomposition, and the finite Weyl group $W_{F}=W_{F}(\mathfrak{g}, \mathfrak{h})$ is reduced to $\{1\}$. In particular, a functional $\lambda \in \mathfrak{h}^{*}$ is classically cohomologically finite if and only if it is dominant.

Proof. Look at the levels where $r_{i}+s_{i}>1$. In the inverse system (5.10) each root is the restriction of several roots, so $\Delta$ is uncountable.

Let $w_{\mathfrak{h}} \in W$ and let $n$ be sufficiently large so that, if $m \geqq n$, then $w$ preserves $\mathfrak{g}_{m}$ and acts by an inner automorphism on $\mathfrak{g}_{m}$. Write this inner automorphism as $w_{m}=\operatorname{Ad}\left(g_{m}\right)$ with $g_{m} \in G_{m}$. Decompose the Borel subalgebras $\mathfrak{b}_{m}=\left(\mathfrak{b} \cap \mathfrak{g}_{m}\right) \subset$ $\mathfrak{g}_{m}$ as $\mathfrak{b}_{m}=\mathfrak{b}_{m}^{\prime} \oplus\left(w_{m}\left(\mathfrak{b}_{m}\right) \cap \mathfrak{b}_{m}\right)$, vector space direct sums, in such a way that 
$d \phi_{\ell, m}\left(\mathfrak{b}_{m}^{\prime}\right) \subset \mathfrak{b}_{\ell}^{\prime}$ for $\ell \geqq m \geqq n$. To do this recursively we need only note that

$$
\begin{aligned}
& d \phi_{m+1, m}\left(\mathfrak{b}_{m}^{\prime}\right) \cap\left(w_{m+1}\left(\mathfrak{b}_{m+1}\right) \cap \mathfrak{b}_{m+1}\right) \\
& \quad=d \phi_{m+1, m}\left(\mathfrak{b}_{m}^{\prime}\right) \cap\left(d \phi_{m+1, m}\left(\mathfrak{g}_{m}\right) \cap\left(w_{m+1}\left(\mathfrak{b}_{m+1}\right) \cap \mathfrak{b}_{m+1}\right)\right) \\
& \quad=d \phi_{m+1, m}\left(\mathfrak{b}_{m}^{\prime}\right) \cap\left(d \phi_{m+1, m}\left(\mathfrak{g}_{m}\right) \cap\left(w_{m+1}\left(\mathfrak{b}_{m+1}\right)\right) \cap\left(d \phi_{m+1, m}\left(\mathfrak{g}_{m}\right) \cap \mathfrak{b}_{m+1}\right)\right) \\
& \quad=d \phi_{m+1, m}\left(\mathfrak{b}_{m}^{\prime}\right) \cap d \phi_{m+1, m}\left(\left(w_{m}\left(\mathfrak{b}_{m}\right) \cap \mathfrak{b}_{m}\right)\right. \\
& \quad=0 .
\end{aligned}
$$

Thus we can use $d \phi_{m+1, m}\left(\mathfrak{b}_{m}^{\prime}\right)$ to start a vector space complement $\mathfrak{b}_{m+1}^{\prime}$ to $w_{m+1}\left(\mathfrak{b}_{m+1} \cap \mathfrak{b}_{m+1}\right)$ in $\mathfrak{b}_{m+1}$.

The classical Weyl group length is $\ell\left(\left.w_{m}\right|_{\mathfrak{h}_{m}}\right)=\operatorname{dim} \mathfrak{b}_{m}^{\prime}$. It is the number of positive root spaces of $\left(\mathfrak{g}_{m}, \mathfrak{h}_{m}\right)$ sent to negative root spaces by $w_{m}$, and $\mathfrak{b}_{m}^{\prime}$ is the sum of those root spaces. By construction of diagonal direct limits, if $\mathfrak{s}$ is a root space of $\left(\mathfrak{g}_{m}, \mathfrak{h}_{m}\right)$ then $d \phi_{m+1, m}(\mathfrak{s})$ is contained in a sum of $s_{m+1}+t_{m+1}$ root spaces of $\left(\mathfrak{g}_{m+1}, \mathfrak{h}_{m+1}\right)$, but not in a sum of fewer. This also shows first that $\mathfrak{g}$ does not have root spaces and second that $\operatorname{dim} \mathfrak{b}_{m+1}^{\prime} \geqq\left(s_{m+1}+t_{m+1}\right) \operatorname{dim} \mathfrak{b}_{m}^{\prime}$. Our direct limit system was assumed to be of non-classical type, so these dimensions are unbounded, unless of course $w=1$. Thus the classical Weyl group lengths $\ell\left(\left.w_{m}\right|_{\mathfrak{h}_{m}}\right)$ are unbounded unless $w=1$, forcing $W_{F}=\{1\}$. The last statement follows.

5.18. Examples of non-classical cohomological finiteness. Let $\mathfrak{g}=\lim _{\longrightarrow} \mathfrak{g}_{n}$ be a diagonal embedding direct limit system (5.1), (5.2) or (5.3) of non-classical type. We give a construction, based on an example of Ivan Dimitrov, for cohomologically finite functionals $\lambda \in \mathfrak{h}^{*}$ that are not classically cohomologically finite.

Consider the case of doubling: $r_{i}=2, s_{i}=t_{i}=0$ for all $i$. Start at some point $n$, set $d=d_{n}$, and let $\lambda_{n}=\sum_{1 \leqq i \leqq d} m_{n, i} \epsilon_{i}$ such that $\lambda_{n}+\rho_{n}$ is nonsingular but not dominant. Thus $\lambda_{n}$ leads to a nontrivial representation of $G_{n}$ on some $H^{k}\left(X_{n} ; \mathcal{O}\left(\mathbb{E}_{n}^{*}\right)\right)$ with $k=q_{n}\left(\lambda_{n}+\rho_{n}\right)>0$. If $G_{n}$ is of Type B, C or D we also assume that each $m_{n, i} \geqq 0$. Let $m_{n}$ be the minimum of the $m_{n, i}$.

We want to construct $\lambda_{n+1}=\sum_{1 \leqq i \leqq 2 d} m_{n+1, i} \epsilon_{i}$ with the restriction condition $\left.\lambda_{n+1}\right|_{\phi_{n+1, n}\left(\mathfrak{h}_{n}\right)}=\lambda_{n}$ and the degree condition $q_{n+1}\left(\lambda_{n+1}+\rho_{n+1}\right)=q_{n}\left(\lambda_{n}+\rho_{n}\right)$. The restriction condition just says $m_{n+1, i}+m_{n+1, d+i}=m_{n, i}$ for $1 \leqq i \leqq d$. View $\lambda_{n}$ as a $d$-tuple $\left(m_{n, 1}, \ldots, m_{n, d}\right)$. The restriction condition then becomes $\lambda_{n+1}=\left(\lambda_{n}-\nu_{n}, \nu_{n}\right)$ for some $d$-tuple $\nu_{n}=\left(b_{n, 1}, \ldots, b_{n, d}\right)$. The degree condition is much more delicate, but it will follow, for example, if

(i) $q_{n}\left(\lambda_{n}-\nu_{n}+\rho_{n}\right)=q_{n}\left(\lambda_{n}+\rho_{n}\right)$

(ii) $q_{n}\left(\nu_{n}+\rho_{n}\right)=0$,

(iii) $\nu_{n}$ satisfies $m_{n}-b_{n, i} \geqq b_{n, i}$.

We can always arrange $(5.19)$ by taking $\nu_{n}=\left(b_{n}, \ldots, b_{n}\right)$ where $m_{n}-b_{n} \geqq b_{n}$ for $\mathfrak{g}$ of Type A, $m_{n}-b_{n} \geqq b_{n} \geqq 0$ for Types BD and C. In any case, we have indeed shown the existence of $\lambda_{n+1} \in \mathfrak{h}_{n+1}^{*}$ with the desired properties, and any string of such $\lambda_{j}$ gives a cohomologically finite element $\lambda \in \mathfrak{h}^{*}=\varliminf_{\varlimsup} \mathfrak{h}_{j}^{*}$ of degree $k=q_{n}\left(\lambda_{n}+\rho_{n}\right)>0$.

This pattern persists beyond doubling. For example, if $r_{n+1}=3$ and $s_{n+1}=$ $t_{n+1}=0$ one takes $\lambda_{n+1}=\left(\lambda_{n}-\mu_{n}-\nu_{n}, \mu_{n}, \nu_{n}\right)$. Then the restriction condition is satisfied, and one can satisfy the degree condition, with the appropriate analogue of 
(5.19). That can always be arranged with $\mu_{n}=\left(a_{n}, \ldots, a_{n}\right)$ and $\nu_{n}=\left(b_{n}, \ldots, b_{n}\right)$ where $m_{n}-\left(a_{n}+b_{n}\right) \geqq a_{n} \geqq b_{n}$ for $\mathfrak{g}$ of Type A, $m_{n}-\left(a_{n}+b_{n}\right) \geqq a_{n} \geqq b_{n} \geqq 0$ for $\mathfrak{g}$ of Type BD or C. But there may be many other possibilities. In any case, the possibility of this construction shows the following:

5.20. Lemma. Let $\mathfrak{g}=\lim _{\mathfrak{g}} \mathfrak{g}_{n}$ be a diagonal embedding direct limit system (5.1), (5.2) or (5.3) of non-classical type. Then there exist cohomologically finite functionals $\lambda \in \mathfrak{h}^{*}$ of arbitrary degree $q_{\lambda}>0$. In particular such $\lambda$ are not classically cohomologically finite.

Finally, as in Section 4, we have the following Bott-Borel-Weil Theorem for diagonal embedding direct limit groups.

5.21. Theorem. Let $\mathfrak{g}=\varliminf_{\mathfrak{g}_{n}}$ be a diagonal embedding direct limit system (5.1), (5.2) or (5.3). Let $\lambda \in \mathfrak{h}^{*}$ be integral so that $\mathbb{E} \rightarrow X$ is well defined.

1. If $\lambda$ is not cohomologically finite then every $H^{q}\left(X ; \mathcal{O}\left(\mathbb{E}^{*}\right)\right)=0$.

2. Assume that $\lambda$ is cohomologically finite of degree $q_{\lambda}$. Then

(i) $H^{q}\left(X ; \mathcal{O}\left(\mathbb{E}^{*}\right)\right)=0$ for $q \neq q_{\lambda}$,

(ii) the inverse limit representation $\pi_{u}^{q_{\lambda}}$ of $G_{u}$ on $H^{q_{\lambda}}\left(X ; \mathcal{O}\left(\mathbb{E}^{*}\right)\right)$ is an irreducible unitary representation,

(iii) if $\mathfrak{g}$ is of classical type, then $\lambda$ is classically cohomologically finite, say by means of $w \in W_{F}$ with $\ell(w)=q_{\lambda}$, and the inverse limit representation $\pi_{u}^{q_{\lambda}}$ of $G_{u}$ on $H^{q_{\lambda}}\left(X ; \mathcal{O}\left(\mathbb{E}^{*}\right)\right)$ has highest weight $\widetilde{\lambda}$ as in Definition 4.5, and

(iv) if $\pi_{u}^{q_{\lambda}}$ satisfies the spectral growth condition (3.4) then $\pi_{u}^{q_{\lambda}}$ extends to a bounded, strongly continuous, holomorphic representation $\pi^{q_{\lambda}}$ of $G$ on $H^{q_{\lambda}}\left(X ; \mathcal{O}\left(\mathbb{E}^{*}\right)\right)$.

Spectral growth. We characterize the cohomologically finite $\lambda \in \mathfrak{h}^{*}$, for which the corresponding irreducible highest weight representation of $G_{u}$ satisfies the spectral growth condition, for the direct limit groups (5.1), (5.2) and (5.3) of classical type.

In the classical situation, write $\lambda \in \mathfrak{h}^{*}$ as $\lambda=\sum_{i=1}^{\infty} m_{i} \epsilon_{i}$ with $\lambda_{n}=d \phi_{n}^{*}(\lambda)=$ $\sum_{i=1}^{\operatorname{dim}\left(\mathfrak{h}_{n}\right)} m_{i} \epsilon_{i}$. Let $\left\{\xi_{i}\right\}$ be the basis of $\mathfrak{h}$ which is dual to $\left\{\epsilon_{i}\right\}$, so $\epsilon_{i}\left(\xi_{j}\right)=\delta_{i, j}$, as before. Write $h \in \mathfrak{h}$ as $h=\sum_{i=1}^{\infty} x_{i} \xi_{i}$. Only finitely many of the $x_{i}$ are non-zero. Then $h_{n}=\sum_{i=1}^{\operatorname{dim}\left(\mathfrak{h}_{n}\right)} x_{i} \xi_{i}$.

5.22. Proposition. Let $G_{u}=\underline{\lim } G_{u, n}$ be a direct limit group (5.1), (5.2) or (5.3) of classical type. Let $\lambda=\sum_{i=1}^{\infty} m_{i} \epsilon_{i}$ be dominant integral. Let $\pi_{u}$ be the irreducible representation of $G_{u}$ with highest weight $\lambda$. Then the following are equivalent:

(i) $\pi_{u}$ satisfies the spectral growth condition,

(ii) $\lim \sup _{i}\left|m_{i}\right|<\infty$, i.e. $\lambda$ is $L_{\infty}$, and

(iii) there exists an index $i_{0}$ such that $m_{i}=m_{j}$ whenever $i, j \geqq i_{0}$.

Proof. Let $n_{0}>0$ such that $\lambda_{n}$ is dominant integral whenever $n \geqq n_{0}$. Identify the Weyl group $W_{n}$ of $G_{n}$ as follows.

(A) $G_{n}=S L(d ; \mathbb{C})$ and $W_{n}$ is the symmetric group $S_{d}$ acting by permutations on $\left\{\epsilon_{1}, \ldots \epsilon_{d}\right\}$.

(BD) $G_{n}=S O(2 d+1 ; \mathbb{C})$ or $G_{n}=S p(d ; \mathbb{C})$, and $W_{n}$ is the extension $F S_{d}$ where $F \cong \mathbb{Z}_{2}^{d}$ acts by sign changes on $\left\{\epsilon_{1}, \ldots \epsilon_{d}\right\}$.

(D) $G_{n}=S O(2 d ; \mathbb{C})$ and $W_{n}$ is the extension $E S_{d}$ where $E \cong \mathbb{Z}_{2}^{d-1}$ acts by even numbers of sign changes on $\left\{\epsilon_{1}, \ldots, \epsilon_{d}\right\}$. 
If $w \in W_{n}$ we write $w\left(\sum m_{i} \epsilon_{i}\right)=\sum m_{w(i)} \epsilon_{i}$, viewing it as acting by (possibly signed) permutation on the coefficients. Now

$$
\limsup _{n \geqq n_{0}}\left(\max _{w_{n} \in W_{n}}\left\{\left|\lambda_{n}\left(w_{n} h_{n}\right)\right|\right\}\right)=\limsup _{n \geqq n_{0}}\left(\max _{w_{n} \in W_{n}}\left\{\left|\sum_{i=1}^{\operatorname{dim}\left(\mathfrak{h}_{n}\right)} m_{w_{n}(i)} x_{i}\right|\right\}\right) .
$$

Proposition 3.14 now shows that $\pi_{u}$ is of spectral growth if and only if

$$
\limsup _{n \geqq n_{0}}\left(\max _{w_{n} \in W_{n}}\left\{\left|\sum_{i=1}^{\operatorname{dim}\left(\mathfrak{h}_{n}\right)} m_{w_{n}(i)} x_{i}\right|\right\}\right)<\infty
$$

for each fixed $h$. This happens if and only if $\lim \sup \left|m_{i}\right|<\infty$.

Since $\lambda$ is dominant integral, the integer sequence $\left\{m_{i}\right\}$ is decreasing. Thus $\lim \sup \left|m_{i}\right|<\infty$ means that $\left\{m_{i}\right\}$ is eventually constant.

In general, diagonal embedding direct limit Lie algebras have roots, but it is easy to see that only those of classical type have root spaces. Compare ([33], [4]). In some special cases, for example the "standard embedding" direct limits, it is of course well known that the limit Lie algebra $\mathfrak{g}$ has both roots and root spaces. There in fact the elements of $\mathfrak{g}$ act as operators of finite rank on $\varliminf_{\longleftarrow}\left\{H^{0}\left(X_{n} ; \mathcal{O}\left(\mathbb{E}_{n}\right)\right), \zeta_{n, m}\right\}$. See Section 6D below.

\section{Some Particular Direct Limit Systems}

In $\S 6 \mathrm{~A}$ we glance at the standard embeddings, the most traditional case of diagonal embeddings. In $\S 6 \mathrm{~B}$ we glance at the Warhol embeddings; they are certain "non-Archimedean" variations on diagonal embeddings, where the index set is not linearly ordered but the method of $\$ 5$ remains valid. The variation is in the ordering of our index set, the set $\mathbb{N}$ of positive integers. In $\S 6 \mathrm{C}$ we look at Escher embeddings, a mild twist (pun intended) on standard embeddings.

6A. Standard Embeddings. The standard embeddings are the traditional special cases of diagonal embeddings, as follows. They are all of classical type.

For Type A we have $r_{i}=1, s_{i}=0$ and $t_{i}=1$. Thus $G_{u, n}=S U(n)$, so $G_{n}=S L(n, \mathbb{C})$. The index set is the natural numbers with the usual ordering. For

$n \geqq m$, the standard embedding $\phi_{n, m}: G_{m} \rightarrow G_{n}$ is specified by $g \mapsto\left(\begin{array}{cc}g & 0 \\ 0 & I_{n-m}\end{array}\right)$ where $I_{n-m}$ is the identity matrix of size $n-m$. The direct limit group $G_{u}$ then consists of infinite matrices of the form $\left(\begin{array}{ll}g & 0 \\ 0 & I\end{array}\right)$ where $g \in G_{u, n}$ for some $n$ and $I$ is an infinite identity matrix. Similarly for the complexified direct limit group $G$. These are the groups which are generally known as $S U(\infty)$ and $S L(\infty, \mathbb{C})$, respectively. We can also use the $U(n)$ and $G L(n ; \mathbb{C})$ and obtain the direct limit groups $U(\infty)$ and $G L(\infty, \mathbb{C})$.

For Type BD we also have $r_{i}=1, s_{i}=0$ and $t_{i}=1$. Thus $G_{u, n}=S O(n)$ and $G_{n}=S O(n, \mathbb{C})$, with the standard embeddings defined as for Type A. The direct limit groups are called $S O(\infty)$ and $S O(\infty, \mathbb{C})$. There are three standard variations here: (i) one can use $G_{u, n}=O(n)$ and $G_{n}=O(n, \mathbb{C})$, (ii) one can take $t_{i}=2$ to stay with Type B or stay with Type D, and (iii) one can do both (i) and (ii). The resulting direct limit groups are usually denoted (i) $O(\infty)$ and $O(\infty, \mathbb{C}$ ), (ii) $S O(2 \infty)$ (resp. $S O(2 \infty+1)$ ) and $S O(2 \infty, \mathbb{C})$ (resp. $S O(2 \infty+1, \mathbb{C})$ ), and (iii) $O(2 \infty)($ resp. $O(2 \infty+1))$ and $O(2 \infty, \mathbb{C})($ resp. $O(2 \infty+1, \mathbb{C}))$. 
For Type $\mathrm{C}$ we again have $r_{i}=1, s_{i}=0$ and $t_{i}=1$. Thus $G_{u, n}=S p(n)$ and $G_{n}=S p(n, \mathbb{C})$ with standard embeddings, as defined above. These direct limit groups are usually denoted $S p(\infty)$ and $S p(\infty, \mathbb{C})$.

6B. Warhol Embeddings. The Warhol embeddings are based on the ordering: $m \preceq n$ if $m$ divides $n$, that is, if $m \mid n$. If $m \preceq n$, we define $\phi_{n, m}: G_{m} \rightarrow G_{n}$ by $\phi_{n, m}(g)=\operatorname{diag}\{g, \ldots, g\}$ with $n / m$ blocks down the diagonal. They are of non-classical type.

As before we have three flavors of direct limit groups: unitary

Type A: $G_{u}=S U_{\text {war }}(\infty)=\lim _{\longrightarrow}\left\{S U(n), \phi_{n, m},(\mathbb{N}, \preceq)\right\}$ and

$$
G=S L_{\mathrm{war}}(\infty ; \mathbb{C})=\underline{\lim }\left\{S L(n ; \mathbb{C}), \phi_{n, m},(\mathbb{N}, \preceq)\right\},
$$

orthogonal

Type BD: $G_{u}=S O_{\text {war }}(\infty)=\varliminf_{\longrightarrow}\left\{S O(n), \phi_{n, m},(\mathbb{N}, \preceq)\right\}$ and

$$
G=S O_{\text {war }}(\infty ; \mathbb{C})=\underline{\lim }\left\{S O(n ; \mathbb{C}), \phi_{n, m},(\mathbb{N}, \preceq)\right\}
$$

and symplectic

$$
\begin{aligned}
\text { Type C: } G_{u}=S p_{\text {war }}(\infty)= & \underset{\lim }{\longrightarrow}\left\{S p(n), \phi_{n, m},(\mathbb{N}, \preceq)\right\} \text { and } \\
& G=S p_{\text {war }}(\infty ; \mathbb{C})=\underline{\lim }\left\{S p(n ; \mathbb{C}), \phi_{n, m},(\mathbb{N}, \preceq)\right\} .
\end{aligned}
$$

As in Proposition 5.9, these systems are set up so that

6.4. Proposition. The Warhol embedding direct limit systems (6.1), (6.2) and (6.3) satisfy the basic alignment conditions (0.1), (0.2) and (0.3). In particular the Borel-Weil Theorem 3.13 applies to those limit groups.

The method of $\S 5$ goes through without change for the Warhol embedding direct limit systems. They are of non-classical type, and have cohomologically finite elements $\lambda \in \mathfrak{h}^{*}$, so the Bott-Borel-Weil Theorem 5.21 holds as stated there.

6C. Escher Embeddings. Consider the classsical groups $U(n)$ and the homomorphisms

$$
\phi_{n+1, n}: U(n) \rightarrow U(n+1) \text { given by } \phi_{n+1, n}(g)=\left(\begin{array}{cc}
g & 0 \\
0 & (\operatorname{det} g)^{-1}
\end{array}\right) .
$$

Setting $\phi_{n, n}=$ id and $\phi_{n+k, n}=\phi_{n+k, n+k-1} \cdots \phi_{n+1, n}$ whenever $k>1$, we obtain a directed system. The Escher embeddings $\phi_{n+1, n}: O(n) \rightarrow O(n+1)$ are defined similarly. They are of classical type.

\section{Coherent Root Orders and Classical Cohomological Finiteness}

In this section we consider direct systems that are the general counterpart for diagonal direct limit systems of classical type with root orders that share the properties of (5.8). We examine classical cohomological finiteness for these "coherent root order" systems and the corresponding Bott-Borel-Weil Theorem.

As before, $\Psi_{\alpha}=\Psi\left(\mathfrak{g}_{\alpha}, \mathfrak{h}_{\alpha}\right)$ denotes the simple root system corresponding to the positive system $\Delta_{\alpha}^{+}=\Delta^{+}\left(\mathfrak{g}_{\alpha}, \mathfrak{h}_{\alpha}\right)$. Write $\perp$ for orthogonality relative to the 
Cartan-Killing form $\langle\cdot, \cdot\rangle_{\beta}$. The first element of our general condition concerns the way simple roots pull back in (0.2): if $\alpha \in A$ there exists $\widetilde{\alpha} \geqq \alpha$ such that

if $\psi_{\alpha} \in \Psi_{\alpha}$ and $\beta \geqq \widetilde{\alpha}$, then there exists $\psi_{\beta} \in \Psi_{\beta}$ such that

$$
\text { (i) } d \phi_{\beta, \alpha}^{*}\left(\psi_{\beta}\right)=\psi_{\alpha} \text { and (ii) } \psi_{\beta}\left(d \phi_{\beta, \alpha}\left(\mathfrak{h}_{\alpha}\right)^{\perp}\right)=0 \text {. }
$$

The second element of our sufficient condition, more or less required by (7.1a)(ii), is that the Cartan-Killing forms are aligned as follows.

$$
\begin{aligned}
& \text { if } \beta \geqq \alpha \text {, there exists } k_{\beta, \alpha} \text { such that } \\
& \qquad k_{\beta, \alpha}\langle\xi, \eta\rangle_{\alpha}=\left\langle d \phi_{\beta, \alpha}(\xi), d \phi_{\beta, \alpha}(\eta)\right\rangle_{\beta} \text { for } \xi, \eta \in \mathfrak{g}_{u, \alpha} .
\end{aligned}
$$

Note that $k_{\beta, \alpha} \geqq 1$ for $\beta \geqq \alpha$ - compute in a basis of $\mathfrak{g}_{u, \beta}$ that starts with a basis of $\mathfrak{g}_{u, \alpha}$. Also, $k_{\gamma, \beta} k_{\beta, \alpha}=k_{\gamma, \alpha}$ for $\gamma \geqq \beta \geqq \alpha$. If $\mathfrak{g}_{u, \alpha}$ is simple then $\langle\cdot, \cdot\rangle_{\beta} \cdot\left(d \phi_{\beta, \alpha} \otimes d \phi_{\beta, \alpha}\right)$ is proportional to $\langle\cdot, \cdot\rangle_{\alpha}$, so $(7.1 \mathrm{~b})$ is automatic.

7.2. Definition. The direct systems $\left\{G_{u, \alpha}, \phi_{\beta, \alpha}\right\}_{\alpha, \beta \in A}$ and $\left\{G_{\alpha}, \phi_{\beta, \alpha}\right\}$ have coherent root orderings if (7.1) holds. (This is the general analogue of (5.8) on classical diagonal direct limit systems.)

7.3. Lemma. If $\left\{G_{u, \alpha}, \phi_{\beta, \alpha}\right\}_{\alpha, \beta \in A}$ have coherent root orderings, then the simple root $\psi_{\beta}$ of (7.1a) is unique. In fact, if $\nu_{\alpha} \in \Delta_{\alpha}$ there is a unique root $\nu_{\beta} \in \Delta_{\beta}$ such that (i) $d \phi_{\beta, \alpha}^{*}\left(\nu_{\beta}\right)=\nu_{\alpha}$ and (ii) $\nu_{\beta}\left(d \phi_{\beta, \alpha}\left(\mathfrak{h}_{\alpha}\right)^{\perp}\right)=0$. In particular there is a root space decomposition $\mathfrak{g}=\mathfrak{h}+\sum_{\nu \in \Delta} \mathfrak{g}_{\nu}$.

Proof. Let $\psi_{\beta}, \psi_{\beta}^{\prime} \in \Psi_{\beta}$ satisfy (7.1a)(i) and (7.1a)(ii). Then they agree on $d \phi_{\beta, \alpha}\left(\mathfrak{h}_{\alpha}\right)$ by (7.1a)(i), and by (7.1a)(ii) they agree on its orthocomplement $d \phi_{\beta, \alpha}\left(\mathfrak{h}_{\alpha}\right)^{\perp}$ in $\mathfrak{h}_{\beta}$. So $\psi_{\beta}=\psi_{\beta}^{\prime}$, uniqueness on the simple root level.

Let $\nu_{\alpha} \in \Delta_{\alpha}^{+}$and expand it as $\sum n_{i} \psi_{i, \alpha}$ with $\psi_{i, \alpha} \in \Psi_{\alpha}$ and $n_{i}$ integers $\geqq 0$. Consider a chain $\left\{\nu_{1, \alpha}, \ldots, \nu_{n, \alpha}\right\} \subset \Delta_{\alpha}^{+}$such that $\nu_{1, \alpha}$ is of the form $\psi_{f(0), \alpha} \in \Psi_{\alpha}$, each $\nu_{m+1, \alpha}-\nu_{m, \alpha}$ is of the form $\psi_{f(m), \alpha} \in \Psi_{\alpha}$, and $\nu_{n, \alpha}=\nu_{\alpha}$. Let $\psi_{i, \beta}$ denote the unique root in $\Psi_{\beta}$ such that $d \phi_{\beta, \alpha}^{*}\left(\psi_{i, \beta}\right)=\psi_{i, \alpha}$ and $\psi_{i, \beta}\left(d \phi_{\beta, \alpha}\left(\mathfrak{h}_{\alpha}\right)^{\perp}\right)=0$. Define $\nu_{1, \beta}=\psi_{f(0), \beta}$, and $\nu_{m+1, \beta}=\nu_{m, \beta}+\psi_{f(m), \beta}$ for $1 \leqq m \leqq n-1$. By (7.1), the integers $\frac{2\langle\nu, \mu\rangle}{\langle\mu, \mu\rangle}$ that determine a chain of roots, in this case that tell us $\nu_{m+1, \alpha}=\nu_{m, \alpha}+\psi_{f(m), \alpha}$ is a root, are the same as those computed for the $\nu_{j, \beta}$. Thus each $\nu_{m+1, \beta}=\nu_{m, \beta}+\psi_{f(m), \beta}$ is a root. Now $\nu_{m, \beta} \in \Delta_{\beta}^{+}$for $1 \leqq m \leqq n$, and $\nu_{\beta}=\nu_{n, \beta}$ satisfies both (i) $d \phi_{\beta, \alpha}^{*}\left(\nu_{\beta}\right)=\nu_{\alpha}$ and (ii) $\nu_{\beta}\left(d \phi_{\beta, \alpha}\left(\mathfrak{h}_{\alpha}\right)^{\perp}\right)=0$. That gives existence on the positive root level. Existence follows for $\nu_{\alpha} \in \Delta_{\alpha}$, and uniqueness follows by the argument used for simple roots.

Lemma 7.3 defines injective maps $d \phi_{\beta, \alpha, *}: \Delta_{\alpha} \rightarrow \Delta_{\beta}$. We define finite root systems, positive finite root systems and simple finite root systems for $(\mathfrak{g}, \mathfrak{h})$ by

$$
\begin{aligned}
\Delta_{F}=\Delta_{F}(\mathfrak{g}, \mathfrak{h}) & =\lim _{F}\left(\Delta\left(\mathfrak{g}_{\alpha}, \mathfrak{h}_{\alpha}\right), d \phi_{\beta, \alpha, *}\right), \\
\Delta_{F}^{+}=\Delta_{F}^{+}(\mathfrak{g}, \mathfrak{h}) & =\lim _{F}\left(\Delta^{+}\left(\mathfrak{g}_{\alpha}, \mathfrak{h}_{\alpha}\right), d \phi_{\beta, \alpha, *}\right), \\
\Psi_{F} & =\Psi_{F}(\mathfrak{g}, \mathfrak{h})=\underline{\longrightarrow}\left(\Psi\left(\mathfrak{g}_{\alpha}, \mathfrak{h}_{\alpha}\right), d \phi_{\beta, \alpha, *}\right) .
\end{aligned}
$$

7.5. Lemma. Let $\left\{G_{u, \alpha}, \phi_{\beta, \alpha}\right\}_{\alpha, \beta \in A}$ have coherent root orderings. Let $\alpha \in A$ and $\beta \in B$ with $\alpha \leqq \widetilde{\alpha} \leqq \beta$.

1. If $\psi_{\alpha} \in \Psi_{\alpha}$ and $\psi_{\beta}=d \phi_{\beta, \alpha, *}\left(\psi_{\alpha}\right)$, then the simple root reflections satisfy $s_{\psi_{\beta}} \cdot d \phi_{\beta, \alpha}=d \phi_{\beta, \alpha} \cdot s_{\psi_{\alpha}}$.

2. Let $w_{\alpha} \in W\left(\mathfrak{g}_{\alpha}, \mathfrak{h}_{\alpha}\right)$. Choose a reduced (minimal length) expression $w_{\alpha}=$ $s_{\psi_{i_{1}, \alpha}} \ldots s_{\psi_{i_{k}, \alpha}}$ where the $\psi_{i_{j}, \alpha} \in \Psi_{\alpha}$. Let $\psi_{i_{j}, \beta}=d \phi_{\beta, \alpha, *}\left(\psi_{i_{j}, \alpha}\right) \in \Psi_{\beta}$. Then 
$w_{\beta}=s_{\psi_{i_{1}, \beta}} \ldots s_{\psi_{i_{k}, \beta}} \in W\left(\mathfrak{g}_{\beta}, \mathfrak{h}_{\beta}\right)$ is a reduced expression. In particular $\ell\left(w_{\alpha}\right)=$ $\ell\left(w_{\beta}\right)$. Furthermore, $w_{\beta}$ is the unique element of $W_{\beta}$ such that (i) $w_{\beta}$ is the identity on $d \phi_{\beta, \alpha}\left(\mathfrak{h}_{\alpha}\right)^{\perp}$ and (ii) $w_{\beta} \cdot d \phi_{\beta, \alpha}=d \phi_{\beta, \alpha} \cdot w_{\alpha}$.

Proof. The hypothesis (7.1b) says that the hyperplane $\left(\psi_{\beta}=0\right)$ in $\mathfrak{h}_{\beta}$ is the orthogonal direct sum $d \phi_{\beta, \alpha}\left(\left(\psi_{\alpha}=0\right)\right) \oplus d \phi_{\beta, \alpha}\left(\mathfrak{h}_{\alpha}\right)^{\perp}$, and that $d \phi_{\beta, \alpha}$ maps the line $\left(\psi_{\alpha}=\right.$ $0)^{\perp}$ in $\mathfrak{h}_{\alpha}$ onto the line $\left(\psi_{\beta}=0\right)^{\perp}$ in $\mathfrak{h}_{\beta}$. It follows that $s_{\psi_{\beta}} \cdot d \phi_{\beta, \alpha}=d \phi_{\beta, \alpha} \cdot s_{\psi_{\alpha}}$. The assertion $w_{\beta} \cdot d \phi_{\beta, \alpha}=d \phi_{\beta, \alpha} \cdot w_{\alpha}$ follows.

We check that the defining expression for $w_{\beta}$ is reduced. The length $\ell\left(w_{\beta}\right) \leqq k$ because of the existence of this expression. If the expression for $w_{\beta}$ were to reduce further, it would do so in a way that reduces the length of the expression for $w_{\alpha}$. See [7, Ex. 1(a), p. 37]. Thus $\ell\left(w_{\beta}\right) \geqq \ell\left(w_{\alpha}\right)=k$ because the expression of $w_{\alpha}$ is reduced.

Let $w_{\beta}^{\prime} \in W_{\beta}$ such that (i) $w_{\beta}^{\prime}$ is the identity on $d \phi_{\beta, \alpha}\left(\mathfrak{h}_{\alpha}\right)^{\perp}$ and (ii) $w_{\beta}^{\prime} \cdot d \phi_{\beta, \alpha}=$ $d \phi_{\beta, \alpha} \cdot w_{\alpha}$. Here (ii) says that $w_{\beta}$ and $w_{\beta}^{\prime}$ agree on $d \phi_{\beta, \alpha}\left(\mathfrak{h}_{\alpha}\right)$, and (i) says that they agree on its orthocomplement $d \phi_{\beta, \alpha}\left(\mathfrak{h}_{\alpha}\right)^{\perp}$. Thus $w_{\beta}^{\prime}=w_{\beta}$.

Lemma 7.5 defines injective maps $\operatorname{Ad}\left(d \phi_{\beta, \alpha}\right): W_{\alpha} \rightarrow W_{\beta}$. Part of Definition 4.4 can be reformulated

$$
\begin{aligned}
& W_{F}=W_{F}(\mathfrak{g}, \mathfrak{h})=\underset{\lim }{\longrightarrow}\left\{W_{\alpha}, \operatorname{Ad}\left(d \phi_{\beta, \alpha}\right)\right\}, \\
& \text { group of linear transformations of } \mathfrak{h} .
\end{aligned}
$$

Denote

$$
\left.d \phi_{\alpha}\left(\mathfrak{h}_{\alpha}\right)^{\perp}:={\underset{\beta}{\beta \geqq \widetilde{\alpha}}}_{\lim _{\beta, \alpha}}\left\{d \phi_{\alpha}\right)^{\perp}, d \phi_{\gamma, \beta}\right\} .
$$

If $w \in W_{F}(\mathfrak{g}, \mathfrak{h})$, say $w=\operatorname{Ad}\left(d \phi_{\alpha}\right)\left(w_{\alpha}\right)$, then $w$ preserves $d \phi_{\alpha}\left(\mathfrak{h}_{\alpha}\right)$, and $w$ is the unique element of $W_{F}(\mathfrak{g}, \mathfrak{h})$ such that (i) $w$ is the identity on $d \phi_{\alpha}\left(\mathfrak{h}_{\alpha}\right)^{\perp}$ and (ii) $w \cdot d \phi_{\alpha}=d \phi_{\alpha} \cdot w_{\alpha}$. Thus

$$
\begin{aligned}
& W_{F}(\mathfrak{g}, \mathfrak{h}) \text { is generated by the simple root reflections } \\
& s_{\psi}: \mathfrak{h} \rightarrow \mathfrak{h} \text { with } \psi \in \Psi_{F}(\mathfrak{g}, \mathfrak{h}) .
\end{aligned}
$$

Note that (7.6) and (7.7b) are consistent with the more general Definition 4.4 of finite Weyl group. Also, here, Lemma 7.5 says that the length $\ell(w)$ of a reduced expression in simple root reflections is the same as $\ell\left(w_{\alpha}\right)$.

On occasion we will want to consider the addition to (7.1b) given by

$$
\text { if } \alpha \in A \text { then } k_{\alpha}:=\limsup _{\beta \geqq \alpha} k_{\beta, \alpha}<\infty \text {. }
$$

When (7.8) holds we automatically have $k_{\alpha} \geqq 1$ and $k_{\beta} k_{\beta, \alpha}=k_{\alpha}$. But more to the point, (7.8) specifies a Cartan-Killing form on $\mathfrak{g}$,

$$
\left\langle\phi_{\alpha}\left(\xi_{\alpha}\right), \phi_{\alpha}\left(\eta_{\alpha}\right)\right\rangle:=k_{\alpha}\left\langle\xi_{\alpha}, \eta_{\alpha}\right\rangle_{\alpha} \text { for } \xi_{\alpha}, \eta_{\alpha} \in \mathfrak{g}_{\alpha} .
$$

The interpretation (7.7) of $W_{F}(\mathfrak{g}, \mathfrak{h})$ as the group of isometries of $\mathfrak{h}$ generated by the simple root reflections, is made explicit when (7.8) holds. For then $s_{\psi}: \mathfrak{h} \rightarrow \mathfrak{h}$ is given by $s_{\psi}(\eta)=\eta-\frac{2\left\langle h_{\psi}, \eta\right\rangle}{\left\langle h_{\psi}, h_{\psi}\right\rangle} h_{\psi}$. Here $\mathfrak{h}_{\psi} \in \mathfrak{h}$ is, of course, defined by $\left\langle h_{\psi}, \eta\right\rangle=\psi(\eta)$.

The argument for (i) $\Leftrightarrow$ (ii) in Proposition 5.15 extends essentially without change to direct systems with coherent root orders, as follows. 
7.10. Proposition. Let $\left\{G_{u, \alpha}, \phi_{\beta, \alpha}\right\}_{\alpha, \beta \in A}$ have coherent root orderings. Let $\lambda \in$ $\mathfrak{h}^{*}$. Then $\lambda$ is cohomologically finite if and only if it is classically cohomologically finite.

Now, as in $\S \S 4$ and 5 , we have

7.11. Theorem. Let $\left\{G_{u, \alpha}, \phi_{\beta, \alpha}\right\}_{\alpha, \beta \in A}$ have coherent root orderings. Let $\lambda \in \mathfrak{h}^{*}$ be integral, so that $\mathbb{E} \rightarrow X$ is well defined.

1. If $\lambda$ is not cohomologically finite then every $H^{q}\left(X ; \mathcal{O}\left(\mathbb{E}^{*}\right)\right)=0$.

2. Assume that $\lambda$ is cohomologically finite of degree $q_{\lambda}$, so also $\lambda$ is classically cohomologically finite, say by means of $w \in W_{F}(\mathfrak{g}, \mathfrak{h})$ with $\ell(w)=q_{\lambda}$. Then

(i) $H^{q}\left(X ; \mathcal{O}\left(\mathbb{E}^{*}\right)\right)=0$ for $q \neq q_{\lambda}$,

(ii) the inverse limit representation $\pi_{u}^{q_{\lambda}}$ of $G_{u}$ on $H^{q_{\lambda}}\left(X ; \mathcal{O}\left(\mathbb{E}^{*}\right)\right)$ is an irreducible unitary representation of highest weight $\widetilde{\lambda}$ as in Definition 4.5 , and

(iii) if $\pi_{u}^{q_{\lambda}}$ satisfies the spectral growth condition (3.4), then $\pi_{u}^{q_{\lambda}}$ extends to a bounded, strongly continuous, holomorphic representation $\pi^{q_{\lambda}}$ of $G$ on $H^{q_{\lambda}}\left(X ; \mathcal{O}\left(\mathbb{E}^{*}\right)\right)$.

\section{Incoherent Root Orders and Cohomological Finiteness}

In this section we return to our general direct limit situation, requiring only the conditions (0.1), (0.2) and (0.3). (In fact we can drop (0.3) if we take the $\mathfrak{q}_{\alpha} \subset \mathfrak{g}_{\alpha}$ to be Borel subalgebras.) We pick out the maximal coherent root order part of the inverse system $\left\{\Delta^{+}\left(\mathfrak{g}_{\alpha}, \mathfrak{h}_{\alpha}\right)\right\}$ and show how it specifies the finite Weyl group $W_{F}(\mathfrak{g}, \mathfrak{b}, \mathfrak{h})$. We then define classical direct limits, extending the notion of "classical" for diagonal direct limits, show that coherent root orders occur only in this classical case, and show in the classical case that the notions of cohomological finiteness and classical cohomological finiteness are the same. This gives an extension of the Bott-Borel-Weil Theorem 7.11.

As before, $\Psi_{\alpha}$ denotes the simple root system that defines the positive root system $\Delta_{\alpha}^{+}=\Delta^{+}\left(\mathfrak{g}_{\alpha}, \mathfrak{h}_{\alpha}\right), \mathfrak{b}_{\alpha}$ is the corresponding Borel subalgebra of $\mathfrak{g}_{\alpha}$, and $\mathfrak{b}$ is the limit Borel in $\mathfrak{g}$. Define

$$
\begin{array}{r}
\Xi_{\alpha}=\left\{\psi_{\alpha} \in \Psi_{\alpha} \mid \text { if } \beta>\alpha \text { then there is a unique } \psi_{\beta} \in \Psi_{\beta}\right. \\
\text { such that } \left.d \phi_{\beta, \alpha}^{*} \psi_{\beta}=\psi_{\alpha}\right\}, \\
W_{\Xi}\left(\mathfrak{g}_{\alpha}, \mathfrak{h}_{\alpha}\right):=\text { subgroup of } W\left(\mathfrak{g}_{\alpha}, \mathfrak{h}_{\alpha}\right) \text { generated by reflections } \\
\text { in the roots } \psi_{\alpha} \in \Xi_{\alpha} .
\end{array}
$$

As in (7.4), (7.6) and (7.7) we have

$$
\begin{aligned}
& \Xi=\left(\underline{\lim }_{\longrightarrow} \Xi_{\alpha}\right) \subset \Delta(\mathfrak{g}, \mathfrak{h}) \text { and } W_{\Xi}(\mathfrak{g}, \mathfrak{h})=\lim _{\longrightarrow} W_{\Xi}\left(\mathfrak{g}_{\alpha}, \mathfrak{h}_{\alpha}\right) \\
& \subset W(\mathfrak{g}, \mathfrak{h}), \text { where we define } W_{\Xi}(\mathfrak{g}, \mathfrak{h}):=\text { group generated } \\
& \text { by the simple root reflections } s_{\psi}: \mathfrak{h} \rightarrow \mathfrak{h} \text { with } \psi \in \Xi .
\end{aligned}
$$


If $\mathfrak{g}=\lim _{\mathfrak{1}} \mathfrak{g}_{\alpha}$, diagonal direct limit of classical type, then the root orders (5.7) and (5.8) lead to

$$
\begin{aligned}
& \text { (5.7): } \quad \text { Type } A_{\ell}: \Xi_{n}=\Psi_{n} \text { and } W_{\Xi}\left(\mathfrak{g}_{n}, \mathfrak{h}_{n}\right)=W\left(\mathfrak{g}_{n}, \mathfrak{h}_{n}\right) \\
& =\text { all permutations of }\left\{\epsilon_{1}, \ldots, \epsilon_{\ell+1}\right\} \text {, } \\
& \text { Type } B_{\ell}: \Xi_{n}=\Psi_{n} \backslash\left\{\epsilon_{n}\right\} \text { and } W_{\Xi}\left(\mathfrak{g}_{n}, \mathfrak{h}_{n}\right) \\
& =\text { all permutations of }\left\{\epsilon_{1}, \ldots, \epsilon_{\ell}\right\} \text {, } \\
& \text { Type } C_{\ell}: \Xi_{n}=\Psi_{n} \backslash\left\{2 \epsilon_{n}\right\} \text { and } W_{\Xi}\left(\mathfrak{g}_{n}, \mathfrak{h}_{n}\right) \\
& =\text { all permutations of }\left\{\epsilon_{1}, \ldots, \epsilon_{\ell}\right\} \text {, } \\
& \text { Type } D_{\ell}: \Xi_{n}=\Psi_{n} \backslash\left\{\epsilon_{\ell-1}+\epsilon_{\ell}\right\} \text { and } W_{\Xi}\left(\mathfrak{g}_{n}, \mathfrak{h}_{n}\right) \\
& =\text { all permutations of }\left\{\epsilon_{1}, \ldots, \epsilon_{\ell}\right\} \text {; }
\end{aligned}
$$

(5.8): Types $A_{\ell}, B_{\ell}, C_{\ell}$ and $D_{\ell}$ :

$$
\Xi_{n}=\Psi_{n} \text { and } W_{\Xi}\left(\mathfrak{g}_{n}, \mathfrak{h}_{n}\right)=W\left(\mathfrak{g}_{n}, \mathfrak{h}_{n}\right) .
$$

Here we can reformulate Proposition 5.14 as

$$
\begin{aligned}
& \text { for both root orders }(5.7) \text { and }(5.8) \text {, } \\
& \text { the finite Weyl group } W_{F}=W_{\Xi}(\mathfrak{g}, \mathfrak{h}) \text {. }
\end{aligned}
$$

Now we can characterize the finite Weyl group in some generality. The proof proceeds as in the proof of (7.6), with $\Xi$ in place of $\Psi$.

8.4. Proposition. The finite Weyl group $W_{F}(\mathfrak{g}, \mathfrak{b}, \mathfrak{h})=W_{\Xi}(\mathfrak{g}, \mathfrak{h})$ as specified in (7.13) above.

A diagonal direct limit system is of classical type, as in Definition 5.11, if and only if it is of classical type in the following more general sense.

8.5. Definition. The direct systems $\left\{G_{\alpha}, \phi_{\beta, \alpha}\right\}$ and $\left\{\mathfrak{g}_{\alpha}, d \phi_{\beta, \alpha}\right\}$ are of classical type if, for every index $\alpha$ sufficiently large and every root $\nu_{\alpha} \in \Delta\left(\mathfrak{g}_{\alpha}, \mathfrak{h}_{\alpha}\right)$, if $\beta \geqq \alpha$ is sufficiently large then there is a unique root $\nu_{\beta} \in \Delta\left(\mathfrak{g}_{\beta}, \mathfrak{h}_{\beta}\right)$ such that $d \phi_{\beta, \alpha}^{*}\left(\nu_{\beta}\right)=\nu_{\alpha}$.

In view of Lemma 7.3,

8.6. Lemma. If $\left\{G_{\alpha}, \phi_{\beta, \alpha}\right\}$ has coherent root orderings then it is of classical type.

The argument that (i) implies (ii) for root order (5.7) in Proposition 5.15 extends to our situation, proving

8.7. Proposition. Let $\left\{G_{\alpha}, \phi_{\beta, \alpha}\right\}$ be of classical type. Then $\lambda \in \mathfrak{h}^{*}$ is cohomologically finite if and only if it is classically cohomologically finite.

This leads to the exact analogue of Theorem 7.11:

8.8. Theorem. Let $\left\{G_{u, \alpha}, \phi_{\beta, \alpha}\right\}_{\alpha, \beta \in A}$ be of classical type. Let $\lambda \in \mathfrak{h}^{*}$ be integral, so that $\mathbb{E} \rightarrow X$ is well defined.

1. If $\lambda$ is not cohomologically finite then every $H^{q}\left(X ; \mathcal{O}\left(\mathbb{E}^{*}\right)\right)=0$.

2. Assume that $\lambda$ is cohomologically finite of degree $q_{\lambda}$, so also $\lambda$ is classically cohomologically finite, say by means of $w \in W_{F}(\mathfrak{g}, \mathfrak{h})$ with $\ell(w)=q_{\lambda}$. Then

(i) $H^{q}\left(X ; \mathcal{O}\left(\mathbb{E}^{*}\right)\right)=0$ for $q \neq q_{\lambda}$,

(ii) the inverse limit representation $\pi_{u}^{q_{\lambda}}$ of $G_{u}$ on $H^{q_{\lambda}}\left(X ; \mathcal{O}\left(\mathbb{E}^{*}\right)\right)$ is an irreducible unitary representation of highest weight $\widetilde{\lambda}$ as in Definition 4.5., and 
(iii) if $\pi_{u}^{q_{\lambda}}$ satisfies the spectral growth condition (3.4), then $\pi_{u}^{q_{\lambda}}$ extends to a bounded, strongly continuous, holomorphic representation $\pi^{q_{\lambda}}$ of $G$ on $H^{q_{\lambda}}\left(X ; \mathcal{O}\left(\mathbb{E}^{*}\right)\right)$.

\section{Appendix A. Direct and Inverse Limits: Basic FaCts}

For most results on direct and inverse limits, the simplest and most efficient proofs are obtained by using the universal mapping properties that define those limits. We use this approach while minimizing categorical language. Thus we define direct and inverse limits by their universal mapping properties, but describe them in less abstract terms for the categories considered in this paper. The index set is not assumed countable in Appendices A and B.

The following definitions are elucidated by commutative diagrams (A.5) and (A.6). The horizontal arrows of (A.5) designate a direct system and the dotted arrows in (A.5) show a compatible family of morphisms.

A.1. Definition. Let $\mathcal{C}$ be a category and $A$ a directed set. A direct system $\left\{S_{\alpha}, \phi_{\beta, \alpha}\right\}_{\alpha, \beta \in A}$ in $\mathcal{C}$ indexed by $A$, consists of assignments of (i) an object $S_{\alpha}$ of $\mathcal{C}$ for every $\alpha \in A$ and (ii) a morphism (arrow) $\phi_{\beta, \alpha}$ of $\mathcal{C}$ from $S_{\alpha}$ to $S_{\beta}$ whenever $\alpha \leqq \beta$ in $A$, such that (a) each $\phi_{\alpha, \alpha}$ is the identity morphism and (b) $\phi_{\gamma, \beta} \cdot \phi_{\beta, \alpha}=\phi_{\gamma, \alpha}$ whenever $\alpha \leqq \beta \leqq \gamma$ in $A$.

For all the categories we consider in this paper, one can define the concept of a strict direct system, as a direct system $\left\{S_{\alpha}, \phi_{\beta, \alpha}\right\}_{\alpha, \beta \in A}$ such that $\alpha \leqq \beta$ in $A$ implies $S_{\alpha} \cong \phi_{\beta, \alpha}\left(S_{\alpha}\right)$ where the latter has the (algebraic, topological or analytic) structure that it inherits from $S_{\beta}$.

A.2. Definition. Let $\left\{S_{\alpha}, \phi_{\beta, \alpha}\right\}_{\alpha, \beta \in A}$ be a direct system in $\mathcal{C}$. Let $T$ be an object of $\mathcal{C}$. Fix an index $\delta \in A$ and a family $\left\{f_{\alpha}\right\}_{\alpha \in A, \delta \leqq \alpha}$, where $f_{\alpha}$ is a morphism in $\mathcal{C}$, from $S_{\alpha}$ to $T$, for each $\alpha \geqq \delta$. Then the family $\left\{f_{\alpha}\right\}_{\alpha \in A}$, is called compatible if $f_{\beta} \cdot \phi_{\beta, \alpha}=f_{\alpha}$ whenever $\delta \leqq \alpha \leqq \beta$ in $A$.

A.3. Definition. Let $\left\{S_{\alpha}, \phi_{\beta, \alpha}\right\}_{\alpha, \beta \in A}$ be a direct system in $\mathcal{C}$. Then its direct limit in $\mathcal{C}$ is a pair $\left(S,\left\{\phi_{\alpha}\right\}_{\alpha \in A}\right)$ where $S$ is an object of $\mathcal{C}$ and $\phi_{\alpha}$ is a morphism in $\mathcal{C}$ from $S_{\alpha}$ to $S$ such that

$$
\phi_{\beta} \cdot \phi_{\beta, \alpha}=\phi_{\alpha} \text { whenever } \alpha \leqq \beta \text { in } A
$$

and

for every object $T$ of $\mathcal{C}$ and every compatible family of morphisms $f_{\alpha}$ in $\mathcal{C}$ from $S_{\alpha}$ to $T$ there is a unique morphism $f$ from $S$ to $T$ in $\mathcal{C}$ such that $f \cdot \phi_{\alpha}=f_{\alpha}$ for all $\alpha \geqq \delta \in A$.

The direct limit of $\left\{S_{\alpha}, \phi_{\beta, \alpha}\right\}_{\alpha, \beta \in A}$ in $\mathcal{C}$ is usually denoted

$$
\left(S,\left\{\phi_{\alpha}\right\}_{\alpha \in A}\right)=\underline{\lim }\left\{S_{\alpha}, \phi_{\beta, \alpha}\right\}_{\alpha, \beta \in A} \text { or simply } S=\underline{\lim } S_{\alpha} .
$$

The morphism $f$ of (A.4b) is called the direct limit of $\left\{f_{\alpha}\right\}_{\alpha \in A}$, denoted $f=\lim _{\longrightarrow} f_{\alpha}$.

The solid arrows of commutative diagram (A.5) show a direct system and its direct limit, while the dashed arrow denotes the direct limit of the compatible family $\left\{f_{\alpha}\right\}$.

It is usual to refer to $S$ as the direct limit of $\left\{S_{\alpha}, \phi_{\beta, \alpha}\right\}_{\alpha, \beta \in A}$ and to write $S=\lim _{\longrightarrow} S_{\alpha}$. We'll follow this standard abuse of notation, but it is important to remember that the limit depends on the $\phi_{\beta, \alpha}$ as well as the $S_{\alpha}$. 


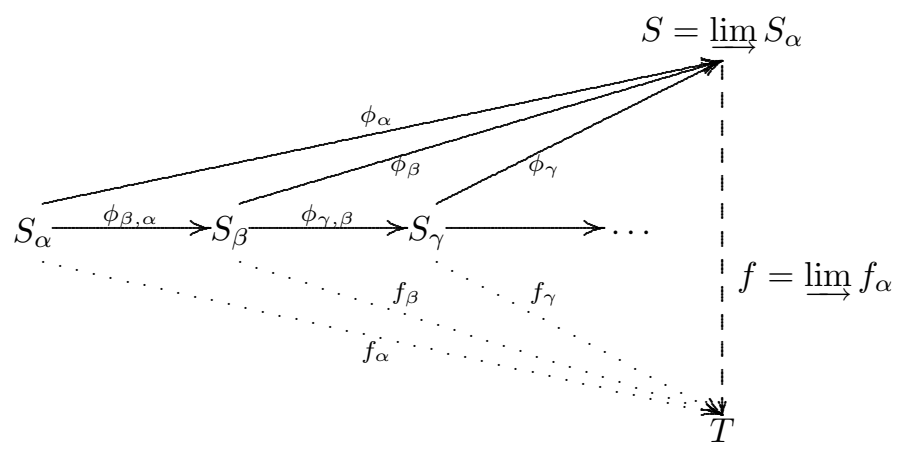

The concepts of inverse system and inverse limit are dual to these: they are obtained by turning backwards all arrows in (A.1), (A.2) and (A.3).

In commutative diagram A.6, the solid arrows designate an inverse system and its inverse limit, the dotted arrows denote a compatible family of morphisms and the dashed arrow denotes the inverse limit of the compatible family $\left\{g_{\alpha}\right\}_{\alpha \in A}$, i.e. the unique morphism whose existence is guaranteed by the universal mapping property of the inverse system.

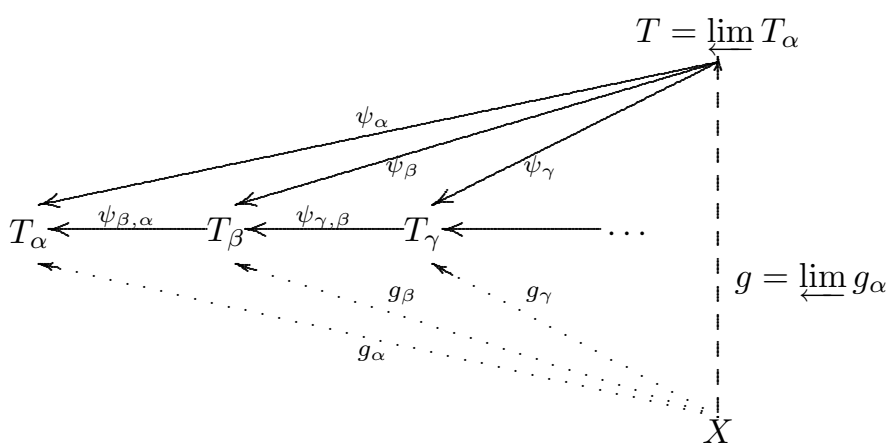

Following the standard abuse of notation, we usually replace $\left(T,\left\{\psi_{\alpha}\right\}_{\alpha \in A}\right)=$ $\varliminf_{L}\left\{T_{\alpha}, \psi_{\beta, \alpha}\right\}_{\alpha, \beta \in A}$ by the simpler expression $T=\varliminf_{\lfloor}\left\{T_{\alpha}, \psi_{\beta, \alpha}\right\}_{\alpha, \beta \in A}$, or even by $T=\varliminf_{\lim } T_{\alpha}$.

In the category of groups, the direct limit $\left(J,\left\{\phi_{\alpha}\right\}_{\alpha \in A}\right)$ of a direct system $\left\{J_{\alpha}, \phi_{\beta, \alpha}\right\}_{\alpha, \beta \in A}$ is constructed as follows. An element of the group $J$ is an equivalence class $\left[j_{\alpha}\right]$ of sets $\left\{j_{\alpha}\right\}$ where each $j_{\alpha} \in J_{\alpha}$ and, for some $\beta \in A$, if $\beta \leqq \gamma$ then $j_{\gamma}=\phi_{\gamma, \beta}\left(j_{\beta}\right)$. The equivalence relation is defined by the eventual behavior: $\left\{j_{\alpha}\right\} \sim\left\{{ }^{\prime} j_{\alpha}\right\}$ if and only if, for some $\beta \in A, j_{\gamma}={ }^{\prime} j_{\gamma}$ whenever $\beta \leqq \gamma$. The group operations of $J$ are given by $\left[j_{\alpha}\right] \cdot\left[{ }^{\prime} j_{\alpha}\right]=\left[j_{\alpha} \cdot{ }^{\prime} j_{\alpha}\right]$ and $\left[j_{\alpha}\right]^{-1}=\left[j_{\alpha}^{-1}\right]$. The homomorphisms $\phi_{\beta}: J_{\beta} \rightarrow J$ are defined by

$$
\phi_{\beta}(x)=\left[j_{\gamma}\right] \text { where } j_{\gamma}=\phi_{\gamma, \beta e t a}(x) \text { if } \beta \leqq \gamma \text {; and } j_{\gamma}=1_{J_{\gamma}} \text { otherwise. }
$$


Direct limits in the category of vector spaces and linear maps, in that of Lie algebras and Lie-algebra homomorphisms, and in other algebraic categories are defined similarly.

Given a direct system $\left\{J_{\alpha}, \phi_{\beta, \alpha}\right\}_{\alpha, \beta \in A}$ of topological groups and continuous group homomorphisms, one constructs the group $J$ and the maps $\phi_{\alpha}: J_{\alpha} \rightarrow J$ as in the category of groups. One then gives $J$ the topology where $U \subset J$ is open if and only if $\phi_{\alpha}^{-1}(U)$ is open in $J_{\alpha}$ for every index $\alpha$. (We call this the naïve direct limit topology.) If

(A.7)

$$
\lim _{\longrightarrow}\left(J_{\alpha} \times J_{\alpha}\right)=J \times J \quad \text { where both sides have the naïve direct limit topology, }
$$

then $\left(J,\left\{\phi_{\alpha}\right\}_{\alpha \in A}\right)$ is the direct limit of the given system in the category of topological groups and continuous group homomorphisms.

It is standard that (A.7) is satisfied whenever the index set A is countable. But, as we learned from [17], condition (A.7) can fail in the uncountable case [21. However, Definition A.3 always implies that the natural inclusion $\underline{\lim }\left(J_{\alpha} \times J_{\alpha}\right) \rightarrow$ $J \times J$ is continuous.

The naïve direct limit topology is also used to obtain direct limits in the category of topological vector spaces and continuous linear maps and in that of topological Lie algebras and continuous Lie-algebra homomorphisms, whenever the analogue of (A.7) holds, for example in case the index set is countable. It is also the underlying topology for the direct limit Lie groups in this paper.

The direct limit of Hilbert spaces has a different topology. See Appendix B.

In this paper we work under assumptions which ensure that each of our direct systems of Lie groups has a direct limit that is not only a topological group but also a Lie group. In other words we require the existence of a direct limit in the category of (possibly infinite-dimensional) Lie groups and analytic homomorphisms. The term "infinite-dimensional Lie groups" has been used in many ways. We use it in the rather strict sense of the following definition.

A.8. Definition. A Lie group is a triple $(G, \mathfrak{g}, \exp )$ where (i) $G$ is both a group and a $C^{\omega}$ manifold in such a way that the map $G \times G \rightarrow G$ by $(x, y) \mapsto x y^{-1}$ is separately $C^{\omega}$, (ii) $\mathfrak{g}$ is a topological Lie algebra, (iii) there is an open neighborhood $\mathcal{O}$ of 0 in $\mathfrak{g}$ such that $U=\exp (\mathcal{O})$ is open in $G$ and exp : $\mathcal{O} \rightarrow U$ is a $C^{\omega}$ diffeomorphism, and (iv) exp restricts to the usual Lie group exponential map from any 1-dimensional subalgebra of $\mathfrak{g}$ onto the corresponding 1-parameter subgroup of $G$.

If the index set is countable then the group operations on the direct limit group are jointly analytic.

As in the finite-dimensional case, we usually write $G$ instead of $(G, \mathfrak{g}, \exp )$.

In [24] we stated that a direct system $\left\{J_{\alpha}, \phi_{\beta, \alpha}\right\}_{\alpha, \beta \in A}$ of Lie groups and analytic homomorphisms has a direct limit in the category of Lie groups if there exists a family of continuous representations $\left\{\pi_{\alpha}, V_{\alpha}\right\}_{\alpha \in A}$ which is compatible (in the sense defined by B.6) and satisfies the imaginary spectral growth condition (3.4a). That growth condition was used only for the assertion that

$$
\mathcal{O}=\{\xi \in \mathfrak{g} \mid \iota(\xi)<\pi\} \text { where } \iota(\xi)=\limsup _{\alpha}\left\{|\operatorname{Im} \lambda| \quad \mid \lambda \text { eigenvalue of } d \pi_{\alpha}(\xi)\right\}
$$

is an open neighborhood of 0 in $\mathfrak{g}$. H. Glöckner pointed out that our proof in [24] is incomplete. However, $\mathcal{O}$ is a neighborhood if we use the operator norm growth condition instead: 
A.9. Proposition. Let $\left\{J_{\alpha}, \phi_{\beta, \alpha}\right\}_{\alpha, \beta \in A}$ be a direct system of real (resp. complex) Lie groups and analytic homomorphisms, and let $\left\{\pi_{\alpha}, V_{\alpha}\right\}_{\alpha \in A}$ be a compatible family of of continuous representations that satisfies the operator norm growth condition (3.4b). Then $\mathcal{O}_{2}=\left\{\xi \in \mathfrak{j} \mid\|d \pi(\xi)\|_{o p}<\pi\right\}$ is an open neighborhood of 0 in $\mathfrak{j}$. Furthermore, $\mathcal{O}_{1}=\{\xi \in \mathfrak{j} \mid \iota(\xi)<\pi\}$ contains $\mathcal{O}_{2}$, so it also is a neighborhood of 0 in $\mathbf{j}$.

Proof. The operator norm growth condition (3.4b) says that each $d \pi(\xi)$ with $\xi \in \mathfrak{j}$ is a bounded operator on $V=\varliminf_{N D L} V_{\alpha}$, the direct limit in the category of normed linear spaces. Let $\mathfrak{g l}_{B}(V)$ denote the Lie algebra of bounded linear transformations of $V$ with topology determined by the operator norm $\|\cdot\|_{\text {op }}$. For each $\alpha$ now $\xi \mapsto$ $d \pi\left(d \phi_{\alpha}(\xi)\right)$ defines a continuous Lie algebra homomorphism $d \widetilde{\pi}_{\alpha}: \mathfrak{j}_{\alpha} \rightarrow \mathfrak{g l}_{B}(V)$. Now $d \pi=\lim _{\longrightarrow} d \pi_{\alpha}=\varliminf_{\lim } d \widetilde{\pi}_{\alpha}: \mathfrak{j} \rightarrow \mathfrak{g l}_{B}(V)$ is continuous. Thus $\mathcal{O}_{2}=\{\xi \in \mathfrak{j} \mid$ $\left.\|d \pi(\xi)\|_{\text {op }}<\pi\right\}$ is an open neighborhood of 0 in $\mathfrak{j}$. But $\iota(\xi) \leqq\|d \pi(\xi)\|_{\text {op }}$, so $\mathcal{O}_{2} \subset \mathcal{O}_{1}$.

Remark. In all of our examples, the self-representations satisfy (3.4b).

The sheaf $\mathcal{C}^{\omega}(J)$ of germs of analytic functions on the direct limit group $J$ is defined as follows. If $S$ is open in $J$, then $f: S \rightarrow \mathbb{C}$ is a section of $\mathcal{C}^{\omega}(J)$ over $S$ just when each $f \cdot \phi_{\alpha}: \phi_{\alpha}^{-1}(S) \rightarrow \mathbb{C}$ is $C^{\omega}$. That specifies the presheaf and thus specifies $\mathcal{C}^{\omega}(J)$. Since the ingredients of this construction are invariant under group operations, the sheaf $\mathcal{C}^{\omega}(J)$ is invariant under left and right group translations and group inversion. Similarly, in the complex analytic case, the sheaf $\mathcal{O}(J)$ of germs of holomorphic functions on $J$ consists of the germs of local functions $f: S \rightarrow \mathbb{C}$ such that each $f \cdot \phi_{\alpha}: \phi_{\alpha}^{-1}(S) \rightarrow \mathbb{C}$ is holomorphic. Although the compatible family of representations $\left\{\pi_{\alpha}\right\}$ is essential for construction of the real or complex analytic structure on the limit group $J$, we have

A.10. Theorem. Let $\left\{J_{\alpha}, \phi_{\beta, \alpha}\right\}_{\alpha, \beta \in A}$ be a direct system of real (resp. complex) Lie groups and analytic homomorphisms. Assume the system has some compatible family of $\left\{\pi_{\alpha}, V_{\alpha}\right\}_{\alpha \in A}$ of continuous representations that satisfies the operator norm growth condition $(3.4 \mathrm{~b})$. Then the resulting direct limit Lie group structure on $J=\lim _{\longrightarrow}\left\{J_{\alpha}, \phi_{\beta, \alpha}\right\}_{\alpha, \beta \in A}$ is independent, up to real analytic (resp. holomorphic) isomorphism, of the choice of $\left\{\pi_{\alpha}, V_{\alpha}\right\}_{\alpha \in A}$ that satisfies (3.4b).

Proof. Let $\left\{\pi_{\alpha}\right\}_{\alpha \in A}$ and $\left\{\widetilde{\pi}_{\alpha}\right\}_{\alpha \in A}$ be compatible families of representations of $\left\{J_{\alpha}, \phi_{\beta, \alpha}\right\}_{\alpha, \beta \in A}$, each of which satisfies the operator norm growth condition. By Theorem 8.1 of [25], $\left\{\pi_{\alpha}\right\}_{\alpha \in A}$ gives rise to a Lie group $J$ and an analytic homomorphism $\phi_{\alpha}: J_{\alpha} \rightarrow J$ for each index $\alpha$ in $A$, and by Theorem 8.5 of that same paper the pair $\left(J,\left\{\phi_{\alpha}\right\}_{\alpha \in A}\right)$ is a direct limit for $\left\{J_{\alpha}, \phi_{\beta, \alpha}\right\}_{\alpha, \beta \in A}$ in the category of Lie groups and analytic homomorphisms. The family $\left\{\tilde{\pi}_{\alpha}\right\}_{\alpha \in A}$ likewise gives rise to a direct limit $\left(\widetilde{J},\left\{\widetilde{\phi}_{\alpha}\right\}_{\alpha \in A}\right)$.

Since $\left\{\widetilde{\phi}_{\alpha}: J_{\alpha} \rightarrow \widetilde{J}\right\}_{\alpha \in A}$ is a compatible family of analytic homomorphisms, and as $\left(J,\left\{\phi_{\alpha}\right\}_{\alpha \in A}\right)$ is a direct limit for the system, it follows from Definition A.3 that there exists a unique analytic homomorphism $\widetilde{\phi}=\lim _{\underline{\phi}} \widetilde{\phi}_{\alpha}: J \rightarrow \widetilde{J}$ such that $\widetilde{\phi} \cdot \phi_{\alpha}=$ $\widetilde{\phi}_{\alpha}$ for every index $\alpha$. Similarly, there exists a unique analytic homomorphism $\phi=\lim _{\phi_{\alpha}}: \widetilde{J} \rightarrow J$ such that $\phi \cdot \widetilde{\phi}_{\alpha}=\phi_{\alpha}$ for all $\alpha$. The map $\phi \cdot \widetilde{\phi}: J \rightarrow J$ is an analytic homomorphism satisfying $(\phi \cdot \widetilde{\phi}) \cdot \phi_{\alpha}=\phi_{\alpha}$ for every index $\alpha$ in $A$. Thus $\phi \cdot \widetilde{\phi}=\varliminf_{\longrightarrow} \phi_{\alpha}: J \rightarrow J$. Now the identity map on $J$ satisfies $\operatorname{id}_{J} \cdot \phi_{\alpha}=\phi_{\alpha}$ for all 
$\alpha$, so that $\operatorname{id}_{J}=\lim _{\alpha} \phi_{\alpha}: J \rightarrow J$. It follows from the uniqueness of the direct limit map that $\phi \cdot \widetilde{\phi}$ is the identity and hence $J$ is analytically isomorphic to $\widetilde{J}$.

Note. The homomorphisms $\phi_{\alpha}: J_{\alpha} \rightarrow J=\underline{\lim }\left\{J_{\alpha}, \phi_{\beta, \alpha}\right\}_{\alpha, \beta \in A}$ are not uniquely determined. For if $\left(J,\left\{\phi_{\alpha}\right\}_{\alpha \in A}\right)$ is a direct limit for $\left\{J_{\alpha}, \phi_{\beta, \alpha}\right\}_{\alpha, \beta \in A}$, and if $\tau$ is an analytic automorphism of $J$, then the limit group $\left(J,\left\{\tau \cdot \phi_{\alpha}\right\}_{\alpha \in A}\right)$ is another direct limit for $\left\{J_{\alpha}, \phi_{\beta, \alpha}\right\}_{\alpha, \beta \in A}$.

\section{Appendix B. Direct and Inverse Limits: Duality of Representations}

This appendix is to show that, under certain hypotheses, the inverse limit of a compatible family $\left\{\pi_{\alpha}^{*}\right\}_{\alpha \in A}$ of representations is equivalent to the dual of the direct limit of the dual representations $\left\{\pi_{\alpha}\right\}_{\alpha \in A}$. The concepts of direct and inverse limit representations then become virtually interchangeable.

Some properties, such as continuity or holomorphy, can be established more easily for direct limit than for inverse limit representations. On the other hand, some representations, such as those involved in the direct limit Bott-Borel-Weil Theorem, present themselves naturally as inverse limits. The results in this appendix can then be used to show that those inverse limit representations also have those desirable properties.

Duality of the representation spaces. We start with a few words on notation. If $Y$ is a locally convex topological vector space then $Y^{*}$ denotes its topological dual space. This is the strong dual. It has the topology of uniform convergence on bounded subsets of $Y$. If $Y$ is a Hilbert space, this is the usual Hilbert space topology on the dual.

If $f$ is a continuous linear map between topological vector spaces, then $f^{*}$ denotes its adjoint. We write $\left\langle v^{*} \mid u\right\rangle$ for the action of an element $v^{*}$ of $Y^{*}$ on the element $u \in Y$.

First consider direct and inverse limits in the category of Hilbert spaces and partial isometries. A linear map from one Hilbert space to another is a partial isometry if it is an isometry on the orthocomplement of its kernel.

It turns out that the limit Hilbert space ipso facto satisfies a universal mapping condition which at first sight appears stronger than the condition required for the direct or inverse limit by the definition given in Appendix A. This property (see Proposition B.1 (2) below) is the one we used in [26] as the definition of the Hilbert direct limit.

In this category, a direct system $\left\{H_{\alpha}, \eta_{\beta, \alpha}\right\}_{\alpha, \beta \in A}$ is strict if and only if each of the partial isometries $\eta_{\beta, \alpha}$ is injective.

B.1. Proposition. Let $\left\{H_{\alpha}, \eta_{\beta, \alpha}\right\}_{\alpha, \beta \in A}$ be a direct system of Hilbert spaces and injective partial isometries.

1. The direct limit $\left(H,\left\{\eta_{\alpha}\right\}_{\alpha \in A}\right)=\underline{\lim }\left\{H_{\alpha}, \eta_{\beta, \alpha}\right\}_{\alpha, \beta \in A}$ exists in the category of Hilbert spaces and partial isometries.

2. $\left(H,\left\{\eta_{\alpha}\right\}_{\alpha \in A}\right)$ has the following universal mapping property. Let $X$ be a Hilbert space and let $\left\{f_{\alpha}: H_{\alpha} \rightarrow X\right\}_{\alpha \in A}$ be a uniformly bounded compatible family of linear maps. In other words $f_{\beta} \cdot \eta_{\beta, \alpha}=f_{\alpha}$ whenever $\alpha \leqq \beta$ in $A$, and there exists $M>0$ such that $\left\|f_{\alpha}(v)\right\|_{X} \leqq M\|v\|_{H_{\alpha}}$ for all $v \in H_{\alpha}$ and all $\alpha \in A$. Then there is a unique bounded linear map $f=\lim _{\alpha} f_{\alpha}: H \rightarrow X$ such that $f \cdot \eta_{\alpha}=f_{\alpha}$ for all $\alpha \in A$. 
Proof. Let $V$ denote the direct limit $\left(H,\left\{\eta_{\alpha}\right\}_{\alpha \in A}\right)=\lim _{\longrightarrow}\left\{H_{\alpha}, \eta_{\beta, \alpha}\right\}_{\alpha, \beta \in A}$ in the category of vector spaces. The norms of the spaces $H_{\alpha}$ are compatible, i.e., whenever $\alpha \leqq \beta$ in $A$ we have $\|v\|_{H_{\alpha}}=\left\|\eta_{\beta, \alpha}(v)\right\|_{H_{\beta}}$ for all $v \in H_{\alpha}$. Thus we can define a norm $\|$.$\| on V$ by $\|v\|=\left\|\eta_{\alpha}^{-1}(v)\right\|_{\alpha}$ where $\alpha$ is any index large enough that $\eta^{-1}(v) \neq \emptyset$. This is independent of the choice of $\alpha$. Equipped with this norm, $V$ is a pre-Hilbert space. Let $H$ be its Hilbert completion.

If $X$ is any Hilbert space and $\left\{f_{\alpha}: H_{\alpha} \rightarrow X\right\}_{\alpha \in A}$ is any uniformly bounded, compatible family of linear maps, then the map $f=\lim f_{\alpha}: H \rightarrow X$ is defined as follows. If $v \in V$, then $f(v)=f_{\alpha}\left(\eta_{\alpha}^{-1}(v)\right)$, where $\alpha$ is any index large enough so that $\eta_{\alpha}^{-1}(v)$ is not empty. The map $f$ is then extended by continuity to all of $H$. $f$ is well-defined because the family $\left\{f_{\alpha}\right\}$ is compatible. Clearly $f$ is linear.

If $M$ is a constant such that $\left\|f_{\alpha}(v)\right\|_{X} \leqq M\|v\|_{H_{\alpha}}$ for all $v \in H_{\alpha}$ and all indices $\alpha$, then $\|f(v)\|_{X} \leqq M\|v\|_{H}$, in particular $f$ is bounded.

The definition of $f$ implies that $f \cdot \eta_{\alpha}=f_{\alpha}$ for every index $\alpha$. This proves that $H$ enjoys the universal mapping property of condition (2) in Proposition B.1.

To show that $H=\varliminf_{\longrightarrow}\left(H_{\alpha},\left\{\eta_{\alpha}\right\}_{\alpha \in A}\right)$ in our category, it remains to be proved that if each $f_{\alpha}$ is a partial isometry, then $f$ is also a partial isometry. Let $K_{\alpha}$ denote the kernel of $f_{\alpha}$, and let $\widetilde{\eta}_{\beta, \alpha}$ denote the restriction of $\eta_{\beta, \alpha}$ to $K_{\alpha}$. Let $K$ denote the Hilbert space completion of the vector-space direct limit $\lim \left\{K_{\alpha}, \widetilde{\eta}_{\beta, \alpha}\right\}_{\alpha \in A}$. This space can be considered in a natural way as a subspace of $\vec{H}$. Then the $K$ is the kernel of $f$ and $\left.f\right|_{H \ominus K}$ is an isometry. Hence $f$ is a partial isometry, and this completes the proof.

B.2. Theorem. Let $\left\{H_{\alpha}^{*}, \eta_{\beta, \alpha}^{*}\right\}_{\alpha, \beta \in A}$ be an inverse system of Hilbert spaces and surjective partial isometries. Then

1. The inverse limit $\left(H^{*},\left\{\eta_{\alpha}^{*}\right\}_{\alpha \in A}\right)=\varliminf_{\lfloor}\left\{H_{\alpha}^{*}, \eta_{\alpha, \beta}^{*}\right\}_{\alpha, \beta \in A}$ exists in the category of Hilbert spaces and surjective partial isometries,

2. $\left(H^{*},\left\{\eta_{\alpha}^{*}\right\}_{\alpha \in A}\right)$ has the following universal mapping property. Let $X$ be a Hilbert space and let $\left\{f_{\alpha}: X \rightarrow H_{\alpha}^{*}\right\}$ be a uniformly bounded compatible family of linear maps. In other words $\eta_{\beta, \alpha}^{*} \cdot f_{\beta}=f_{\alpha}$ whenever $\alpha \leqq \beta$ in $A$, and there exists $M>0$ such that $\left\|f_{\alpha}(x)\right\|_{H_{\alpha}^{*}} \leqq M\|x\|_{X}$ for all $x \in X$ and all $\alpha \in A$. Then there is a unique bounded linear map $f=\lim f_{\alpha}: X \rightarrow H^{*}$ such that $\eta_{\alpha}^{*} \cdot f=f_{\alpha}$ for all $\alpha \in A$.

3. $\left(\lim _{\longrightarrow} H_{\alpha}\right)^{*} \cong \varliminf_{\lim } H_{\alpha}^{*}$ and $\left(\varliminf H_{\alpha}\right)^{*} \cong \lim _{\longrightarrow} H_{\alpha}^{*}$.

Statement (3) can be expressed more precisely as

$$
\begin{gathered}
\left(H,\left\{\eta_{\alpha}\right\}_{\alpha \in A}\right)=\lim _{\longrightarrow}\left\{H_{\alpha}, \eta_{\beta, \alpha}\right\}_{\alpha, \beta \in A} \text { if and only if } \\
\left(H^{*},\left\{\eta_{\alpha}^{*}\right\}_{\alpha \in A}\right)=\lim _{\longleftrightarrow}\left\{H_{\alpha}^{*}, \eta_{\beta, \alpha}^{*}\right\}_{\alpha, \beta \in A} .
\end{gathered}
$$

Proof. The dual spaces $H_{\alpha}=H_{\alpha}^{* *}$ to the $H_{\alpha}^{*}$, together with the adjoint maps $\eta_{\beta, \alpha}=\eta_{\beta, \alpha}^{* *}$ to the $\eta_{\beta, \alpha}^{*}$, constitute the direct system $\left\{H_{\alpha}, \eta_{\beta, \alpha}\right\}_{\alpha, \beta \in A}$ dual to $\left\{H_{\alpha}^{*}, \eta_{\beta, \alpha}^{*}\right\}_{\alpha, \beta \in A}$. This direct system satisfies the hypotheses of Proposition B.1, hence it has a direct limit $\left(H,\left\{\eta_{\alpha}\right\}_{\alpha \in A}\right)$.

Let $X$ be a Hilbert space and $\left\{f_{\alpha}: X \rightarrow H_{\alpha}^{*}\right\}_{\alpha \in A}$ a uniformly bounded, compatible family of linear maps. Then the diagram (B.3) below is commutative. We will show that there exists a bounded linear map $f: X \rightarrow H^{*}$ such that $\eta_{\alpha}^{*} \cdot f=f_{\alpha}$ for all $\alpha \in A$, in other words such that (B.3) remains commutative when $f: X \rightarrow H^{*}$ is adjoined to it. 


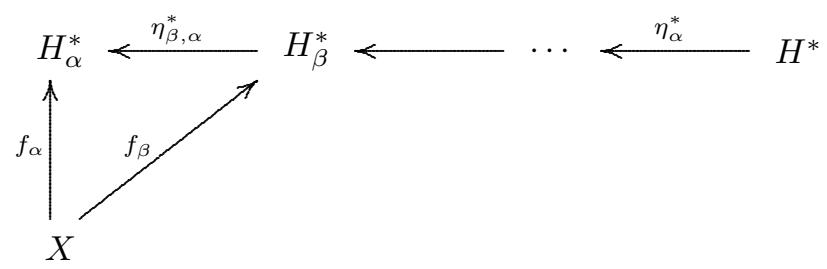

Taking the adjoints of all the maps in (B.3) we obtain

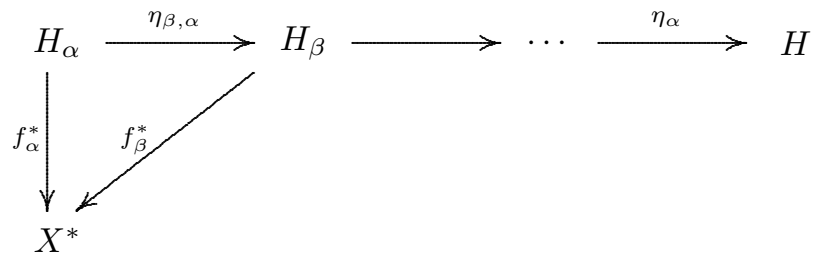

By Proposition B.1 (2), there is a unique bounded linear map $g: H \rightarrow X^{*}$ such that $\eta_{\alpha} \cdot g=f_{\alpha}^{*}$ for each $\alpha \in A$. In other words, adjoining the arrow $g: H \rightarrow X^{*}$ to (B.4) results in a commutative diagram.

The map $f=g^{*}$ has the required properties. If the maps $f_{\alpha}$ are all partial isometries, then $f$ is also a partial isometry. This proves that $H^{*}=\varliminf_{\varliminf}\left\{H_{\alpha}^{*}, \eta_{\beta, \alpha}^{*}\right\}_{\beta, \alpha \in A}$ in our category and that $H^{*}$ satisfies (2). Thus statements (1), (2) and the first half of (3) are proved. Dualize the argument to prove the second half of (3).

B.5. Corollary. Hilbert space direct limits coincide with Hilbert space inverse limits: for any direct system $\left\{H_{\alpha}, \eta_{\beta, \alpha}\right\}_{\alpha, \beta \in A}$ of Hilbert spaces and injective partial isometries we have $\lim _{\longrightarrow} H_{\alpha} \cong \lim _{\longleftrightarrow} H_{\alpha}$.

Proof. Consider the following diagram, where the vertical arrows are obtained using the canonical conjugate-linear isomorphism between a Hilbert space and its dual:

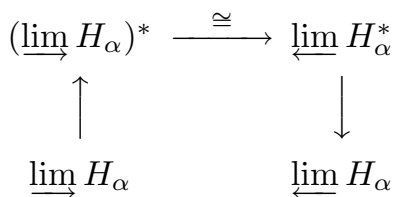

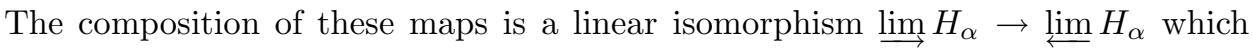
makes the diagram commutative.

Direct limit and inverse limit representations; definitions and duality. Let $\left\{J_{\alpha}, \phi_{\beta, \alpha}\right\}_{\alpha, \beta \in A}$ be a direct system of topological groups and continuous group homomorphisms, and let $J=\lim _{\longrightarrow}\left\{J_{\alpha}, \phi_{\beta, \alpha}\right\}_{\alpha, \beta \in A}$ be its direct limit.

B.6. Definition. A compatible direct family of representations of $\left\{J_{\alpha}, \phi_{\beta, \alpha}\right\}_{\alpha, \beta \in A}$ consists of a direct system $\left\{V_{\alpha}, \eta_{\beta, \alpha}\right\}_{\alpha, \beta \in A}$ of topological vector spaces and continuous linear maps, together with a representation $\pi_{\alpha}$ of $J_{\alpha}$ on $V_{\alpha}$ for each index $\alpha$, which satisfy the compatibility condition:

$$
\pi_{\beta}\left(\phi_{\beta, \alpha} x\right)\left(\eta_{\beta, \alpha} v\right)=\eta_{\beta, \alpha}\left(\pi_{\alpha}(x)(v)\right) \quad \text { for all } x \in J_{\alpha} \text { and all } v \in V_{\alpha}
$$


whenever $\alpha \leqq \beta$ in $A$. The corresponding direct limit representation is the representation $\underline{\lim } \pi_{\alpha}$ of $J$ on $V=\underline{\lim } V_{\alpha}$ given by

$$
\underset{\varliminf}{\longrightarrow} \pi_{\alpha}(x)(u)=\eta_{\alpha}\left(\pi_{\alpha}\left(\phi_{\alpha}^{-1} x\right)\right)\left(\eta_{\alpha}^{-1}(u)\right) \quad(x \in J, u \in V, \alpha \in A, \alpha \gg 0) .
$$

B.8. Definition. A compatible inverse family of representations of $\left\{J_{\alpha}, \phi_{\beta, \alpha}\right\}_{\alpha, \beta \in A}$ consists of an inverse system $\left\{V_{\alpha}^{*}, \eta_{\beta, \alpha}^{*}\right\}_{\alpha, \beta \in A}$ of topological vector spaces and continuous linear maps, together with a representation $\sigma_{\alpha}$ of $J_{\alpha}$ on $V_{\alpha}^{*}$ for each index $\alpha$, which satisfy the compatibility condition:

$$
\eta_{\beta, \alpha}^{*}\left(\sigma_{\beta}\left(\phi_{\beta, \alpha} x\right)\right)\left(v^{*}\right)=\sigma_{\alpha}(x)\left(\eta_{\beta, \alpha}^{*} v^{*}\right) \quad \text { for all } x \in J_{\alpha} \text { and all } v^{*} \in V_{\beta}^{*}
$$

whenever $\alpha \leqq \beta$ in $A$. The corresponding inverse limit representation is the representation $\varliminf_{\alpha} \sigma_{\alpha}$ of $J$ on $V^{*}=\varliminf^{\lim } V_{\alpha}^{*}$ given by

$$
\eta_{\alpha}^{*}\left(\varliminf_{\lim _{\alpha}} \sigma_{\alpha}(x)\left(v^{*}\right)\right)=\sigma_{\alpha}\left(\phi_{\alpha}^{-1} x\right)\left(\eta_{\alpha}^{*} v^{*}\right) \text { for } \alpha \in A, x \in J, v^{*} \in V^{*} .
$$

If $\pi$ is a representation then $\pi^{*}$ denotes the dual (contragredient) representation.

If $\left\{V_{\alpha}, \eta_{\beta, \alpha}\right\}_{\alpha, \beta \in A}$ is a direct system of topological vector spaces and $\left\{\pi_{\alpha}, V_{\alpha}\right\}$ a compatible direct family of representations of $\left\{J_{\alpha}, \phi_{\beta, \alpha}\right\}_{\alpha, \beta \in A}$, then by taking the strong duals $V_{\alpha}^{*}$, the transpose maps $\eta_{\beta, \alpha}^{*}$ and the contragredient representations $\pi_{\alpha}^{*}$, we obtain a compatible inverse family of representations of $\left\{J_{\alpha}, \phi_{\beta, \alpha}\right\}_{\alpha, \beta \in A}$.

Indeed, start from the assumption that $\left\{\pi_{\alpha}\right\}$ satisfies (B.7a). Take any $\alpha$ and $\beta$ with $\alpha \leqq \beta$ in $A$ and fix an element $x \in J_{\alpha}$. Then $\left(\pi_{\beta}\left(\phi_{\beta, \alpha} x\right) \cdot \eta_{\beta, \alpha}\right)(v)=$ $\left(\eta_{\beta, \alpha} \cdot \pi_{\alpha}(x)\right)(v)$ for all $v$ in $V_{\alpha}$. That is to say, $\pi_{\beta}\left(\phi_{\beta, \alpha} x\right) \cdot \eta_{\beta, \alpha}=\eta_{\beta, \alpha} \cdot \pi_{\alpha}(x)$.

Taking transposes we obtain $\eta_{\beta, \alpha}^{*} \cdot\left(\pi_{\beta}\left(\phi_{\beta, \alpha} x\right)\right)^{*}=\left(\pi_{\alpha}(x)\right)^{*} \cdot \eta_{\beta, \alpha}^{*}$. This is equivalent to the condition that $\left(\eta_{\beta, \alpha}^{*} \cdot \pi_{\beta}^{*}\left(\phi_{\beta, \alpha} x^{-1}\right)\right)\left(v^{*}\right)=\left(\pi_{\alpha}^{*}\left(x^{-1}\right) \cdot \eta_{\beta, \alpha}^{*}\right)\left(v^{*}\right)$ for all $v^{*}$ in $V_{\beta}^{*}$.

Since $x \in J_{\alpha}$ was arbitrarily chosen, this shows that $\left\{\pi_{\alpha}^{*}\right\}$ satisfies (B.9a), which proves our claim.

Similarly, if we start with a compatible inverse family of representations and take the contragredients, we obtain a compatible direct family.

B.10. Theorem. Let $\left\{J_{\alpha}, \phi_{\beta, \alpha}\right\}_{\alpha, \beta \in A}$ be a strict direct system of topological groups and continuous group homomorphisms, and let $J$ be its direct limit (as a topological group). Let $\left\{\left(\pi_{\alpha}, H_{\alpha}\right)\right\}_{\alpha \in A}$ denote a compatible direct family of representations of the $J_{\alpha}$ such that $\left\{H_{\alpha}, \eta_{\beta, \alpha}\right\}_{\alpha, \beta \in A}$ is a strict direct system of Hilbert spaces and injective partial isometries. Assume that $\lim _{\alpha} \pi_{\alpha}(x)$ is a bounded operator for each $x \in J$. Then the direct limit representation $\varliminf_{\longrightarrow} \pi_{\alpha}$ and the inverse limit represen-

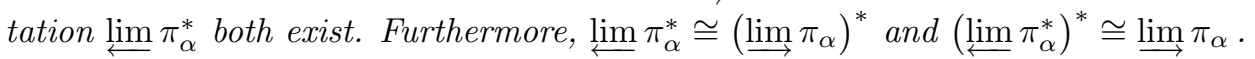

Proof. Existence of the limit representations is given by (B.7b) and (B.9b). Corollary B.5 shows that their representation spaces are naturally isomorphic. Let $V$ be the normed linear space direct limit $\lim _{\longrightarrow} H_{\alpha}$ (as constructed in the proof of Proposition B.1), so $V$ is a pre-Hilbert space whose completion is the Hilbert space direct limit $H$ of the $H_{\alpha}$. We next prove that

$$
\left\langle v^{*} \mid\left(\lim _{\longrightarrow} \pi_{\alpha}(x)\right)(u)\right\rangle=\left\langle\left(\varliminf_{\longleftrightarrow} \pi_{\alpha}^{*}\left(x^{-1}\right)\right) v^{*} \mid u\right\rangle \quad \text { for all } x \in J, u \in H \text { and } v^{*} \in H^{*} .
$$


Indeed, (B.7) and (B.9) show that if $u \in V$ and $v^{*} \in H^{*}$, then

$$
\begin{aligned}
& \left\langle v^{*} \mid\left(\lim _{\longrightarrow} \pi_{\alpha}(x)\right)(u)\right\rangle=\left\langle v^{*} \mid \eta_{\alpha}\left(\pi_{\alpha}\left(\phi_{\alpha}^{-1} x\right)\right)\left(\eta_{\alpha}^{-1} u\right)\right\rangle=\left\langle\eta_{\alpha}^{*} v^{*} \mid \pi_{\alpha}\left(\phi_{\alpha}^{-1} x\right)\left(\eta_{\alpha}^{-1} u\right)\right\rangle \\
& =\left\langle\pi_{\alpha}^{*}\left(\phi_{\alpha}^{-1} x^{-1}\right)\left(\eta_{\alpha}^{*} v^{*}\right) \mid \eta_{\alpha}^{-1} u\right\rangle=\left\langle\eta_{\alpha}^{*}\left(\varliminf_{\longleftarrow} \pi_{\alpha}^{*}\left(x^{-1}\right)\left(v^{*}\right)\right) \mid \eta_{\alpha}^{-1} u\right\rangle \\
& =\left\langle\varliminf_{\longleftarrow} \pi_{\alpha}^{*}\left(x^{-1}\right)\left(v^{*}\right) \mid u\right\rangle \text {. }
\end{aligned}
$$

We now extend by continuity to all $u \in H$ and that completes the proof of $\lim _{\alpha}^{*} \cong$ $\left(\lim _{\alpha} \pi_{\alpha}\right)^{*}$.

To prove the last statement, observe that $\lim _{\alpha} \pi_{\alpha}$ is a unitary representation, and hence it is equivalent to its double contragredient. We thus have that $\left(\stackrel{\lim }{\longleftarrow} \pi_{\alpha}^{*}\right)^{*} \cong$ $\left(\varliminf_{\alpha} \pi_{\alpha}\right)^{* *} \cong \varliminf_{\alpha} \pi_{\alpha}$

\section{REFERENCES}

[1] Bahturin, Yu. A. and Benkart, G., Highest weight modules for locally finite Lie algebras, in "Modular Interfaces" (Proceedings, Riverside, CA, 1995), AMS/IP Studies Adv. Math. 4, Amer. Math. Soc., 1997, 1-31. MR 98m:17009

[2] Bahturin, Yu. A. and Strade, H, Locally finite dimensional simple Lie algebras, Mat. Sbornik 81 (1995), 137-161. MR 95a:17025

[3] Some examples of locally finite simple Lie algebras, Archives Math. 65 (1995), 23-26. MR 96f: 17010

[4] Baranov, A. A., Simple diagonal locally finite Lie algebras, Proc. London Math. Soc. 77 (1998), 362-386. MR 99k:17041

[5] Baranov, A. A. \& Zhilinskii, A. G., Diagonal direct limits of simple Lie algebras, Comm. Algebra 27 (1999), 2749-2766. MR 2000f:17031

[6] Bott, R., Homogeneous vector bundles, Annals of Math. 66 (1957), 203-248. MR 19:681d

[7] Bourbaki, N., Groupes et Algèbres de Lie (Ch. 4, 5 et 6), Éléments de Mathématique, Fasc. XXXIV, Hermann, Paris, 1968. MR 39:1590

[8] Boyer, R. P., Representation theory of the Hilbert-Lie group $U_{2}(H)$, Duke J. Math. 47 (1980), 205-236. MR 81j:22024

[9] Characters and factor representations of the infinite classical groups, J. Operator Theory 28 (1993). MR 96e:22036

[10] , Representation theory of infinite dimensional unitary groups, Contemporary Math. 145 (1993). MR 94g:22038

[11] Dimitrov, I, Private communications, May and June 1999.

[12] Dimitrov, I. \& Penkov, I., Partially integrable highest weight modules, Transformation Groups 3 (1998), 241-253. MR 99j:17035

[13] W Weight modules of direct limit Lie algebras, International Math. Research Notices, No. 5 (1999), 223-249. MR 2000a:17005

[14] Dimitrov, I., Penkov, I. \& J. A. Wolf, A Bott-Borel-Weil theory for direct limits of algebraic groups, to appear.

[15] Eda, K., Kiyosawa, T., and Ohta, H., N-completeness and its applications, in "Topics in General Topology", North-Holland, Amsterdam, 1989, pp. 459-521. MR 91m:54018

[16] Gel'fand, I.M. and Graev, M.I., Principal representations of the group $U(\infty)$, in "Representations of Lie Groups and Related Topics", Adv. Stud. Contemp. Math., vol. 7, Gordon \& Breach, New York, 1990, pp. 119-153. MR 92k:22033

[17] Glöckner, H., Private communications.

[18] Habib, A., Direct limits of Zuckerman derived functor modules, Doctoral Dissertation, University of California, Berkeley, 1997. Journal publication to appear.

[19] Hartshorne, R., On the de Rham cohomology of algebraic varieties, Publ. Math. IHES 45 (1976), 5-99. MR 55:5633

[20] _ Algebraic Geometry, Graduate Texts in Mathematics 52, Springer-Verlag, 1977. MR 57:3116

[21] Kakutani, S. \& Klee, V., The finite topology of a linear space, Arch. Math. 14 (1963), 55-58. MR 27:1799 
[22] Kirillov, A. A., Representations of the infinite unitary group, Soviet Math. Doklady 14 (1973), 288-290. MR 49:5239

[23] Natarajan, L., Unitary highest weight modules of inductive limit Lie algebras and groups, J. Algebra 167 (1994), 9-28. MR 95f:22022

[24] Natarajan, L., Rodríguez-Carrington, E. and Wolf, J. A., Differentiable structure for direct limit groups, Letters in Math. Physics 23 (1991), 101-123. MR 92k:22035

[25] L L Locally convex Lie groups, Nova J. Algebra and Geometry 2 (1993), 59-88. MR 94j:22022

[26] New classes of infinite dimensional groups, "Algebraic Groups and their Generalizations;" Proceedings of Symposia in Pure Mathematics 56, Part II (1994), 377-392. MR 95c:22024

[27] Neretin, Yu. A., Categories of Symmetries and Infinite-Dimensional Groups, Clarendon Press, Oxford, 1996. MR 98b:22003

[28] Ol'shanskii, G. I., Unitary representations of the group $S O_{0}(\infty, \infty)$ as limits of unitary representations of the groups $S_{0}(n, \infty)$ as $n \rightarrow \infty$, Functional Analysis \& Applications 20 (1988), 292-301. MR 88b:22025

[29] — Representations of infinite dimensional classical groups, limits of enveloping algebras, and Yangians, Advances Soviet Math. 2 (1991), 1-66. MR 92g:22039

[30] Pickrell, D., The separable representations of $U(H)$, Proceedings Amer. Math. Soc. 102 (1988), 416-420. MR 89c:22036

[31] Rodríguez-Carrington, E. and Wolf, J. A., Infinite Weyl groups, to appear.

[32] Stratila, S. and Voiculescu, D., "Representations of AF-algebras and of the group $U(\infty)$ ", Lecture Notes in Math. 486 (1975). MR 56:16391

[33] Yanson, I. A. and Zhdanovich, D. V., The set of direct limits of Lie algebras of type A, Communications in Algebra 24 (1996), 1125-1156. MR 97c:17035

[34] Zhdanovich, D. V., Doctoral Dissertation, Moscow State University, 1996.

Department of Mathematics 0112, University of California at San Diego, La Jolla, CALifornia 92093

E-mail address: loki@euclid.ucsd.edu

Department of Mathematics, Rutgers University, 110 Frelinghuysen Rd., Piscataway, NEW JeRsey 08854

E-mail address: carringt@math.rutgers.edu

Department of Mathematics, University of California at Berkeley, Berkeley, CalIFORNIA 94720-3840

E-mail address: jawolf@math.berkeley.edu 